\title{
Center of Brauer Configuration Algebras
}

\author{
Alex Sierra Cárdenas
}

TESE APRESENTADA

$\mathrm{AO}$

Instituto DE MATEMÁtica E EstatísticA

DA

Universidade de SÃo Paulo

PARA

OBTENÇÃO DO TÍTULO

$\mathrm{DE}$

Doutor EM CIÊNCIAS

Programa: Matemática

Orientador: Prof. Dr. Eduardo do Nascimento Marcos

Coorientador: Prof. Dr. Sibylle Schroll

Durante o desenvolvimento deste trabalho o autor recebeu auxílio financeiro da CAPES-PROEX

São Paulo, Agosto de 2017 


\section{Center of Brauer Configuration Algebras}

Esta versão da tese contém as correções e alterações sugeridas pela Comissão Julgadora durante a defesa da versão original do trabalho, realizada em 05/06/2017. Uma cópia da versão original está disponível no

Instituto de Matemática e Estatística da Universidade de São Paulo.

Comissão Julgadora:

- Prof. Dr. Eduardo do Nascimento Marcos (orientador) - IME-USP

- Prof ${ }^{a}$. Dra ${ }^{a}$. Regina Maria de Aquino - UFES

- Prof. Dr. Edson Ribeiro Alvares - UFPR

- Pós-Doc. Iurii Volkov - IME-USP

- Prof. Dr. Edward Lewis Green - Virginia Polytechnic Institute and State University (USA) 


\section{Acknowledgments}

I wish to express my thanks to my adviser, Professor Eduardo, for his teachings, help, support, trust and patience in all the process of this present work. Also I would like to thank to the Postdoc student Iurii Volkov, who has pointed out some mistakes in my exposition and has supplied my work with counterexamples and observations. And I would specially like to thank to my co-adviser, Professor Sibylle Schroll, for her enormous patience, and for providing me with her valuable suggestions, remarks, corrections and time to make this work even better. Thank you very much, Professor Sibylle.

This work is dedicated to my family: my mom, my dad, my brother and my sister. Gracias por estar siempre ahi apoyándome incondicionalmente durante todos estos años, y por enseñarme que debemos estar unidos porque somos poquitos. Los amo bastante. 


\section{Resumo}

SIERRA, A. Centro das Álgebras de Configurção de Brauer. Tese (Doutorado) - Instituto de Matemática e Estatística, Universidade de São Paulo, São Paulo, 2017.

Na presente tese apresentamos uma nova classe the álgebras de dimensão finita que vamos chamar de álgebras de configuração de Brauer. Além de estudar algumas das propriedades básicas de uma algebra de configuração de Brauer, vamos concentrar nosso trabalho em desenvolver as ferramentas necessárias para o cálculo de uma base explícita do centro. Também obtemos uma expressão interessante do valor da dimensão do centro que mostra que esse valor pode-se cacular diretamente dos dados combinatórios da configuração de Brauer.

Palavras-chave: Configuração de Brauer, Álgebra de configuração de Brauer, Centro. 


\section{Abstract}

SIERRA, A. Center of Brauer Configuration Algebras. Doctoral Thesis - Instituto de Matemática e Estatística, Universidade de São Paulo, São Paulo, 2017.

In the present thesis we introduce a new class of finite dimensional algebras that we call Brauer configuration algebras. Besides studying some of the basic properties of a Brauer configuration algebra, we focus our work on developing the necessary tools to calculate an explicit basis of its center. We also obtain an interesting expression for the value of the dimension of the center that shows that it can be directly read off from the combinatorial data of the Brauer configuration.

Keywords: Brauer configuration, Brauer configuration algebra, Center. 


\section{Contents}

$\begin{array}{ll}\text { Introduction } & 1\end{array}$

1 Preliminaries $\quad 5$

1.1 Basic concepts . . . . . . . . . . . . . . . . . . . . . 5

1.2 Quivers and path algebras . . . . . . . . . . . . . . . . . 9

1.3 Brauer configuration algebras . . . . . . . . . . . . . . 12

1.3.1 Brauer configuration . . . . . . . . . . . . . . . 12

1.3.2 Brauer configuration algebras . . . . . . . . . . . . . . . 16

1.3.3 Special cycles . . . . . . . . . . . . . . . . . . . . . . 24

1.3.4 Some properties of Brauer configuration algebras . . . . . . . . . 27

1.3.5 Decomposable and indecomposable Brauer configuration algebras . . 29

1.4 Induced boundary maps on the Hochschild complex . . . . . . . . . . . . . . 29

2 Center and Dimension $\quad 33$

$2.1 \quad$ Basis of $v \Lambda v \ldots \ldots \ldots \ldots \ldots$

2.1.1 Non-special cycles . . . . . . . . . . . . . . . . . . . . . . . . 34

2.1.2 Some characteristic elements . . . . . . . . . . . . . . . . . 38

2.2 Dimension of the center of a Brauer configuration algebra . . . . . . . . . . 43

$\begin{array}{ll}\text { Bibliography } & 57\end{array}$ 
viii CONTENTS 


\section{Introduction}

A finite-dimensional algebra can be classified into one of three representation types: finite, tame and wild. Finite representation type means that there are only finitely many isomorphism classes of indecomposable modules. When an algebra is not of finite representation type then it just can be either of tame representation type or wild representation type [Dro]. An algebra of tame representation type has infinitely many indecomposable modules which in turn are parameterized by one-parameter families. But, when an algebra is of wild representation type there is no way to parameterize the isomorphism classes of the indecomposable modules. So, in the framework of algebras of wild representation type there is little knowledge or understanding of the nature of this phenomenon.

In this thesis we consider a new class of mostly wild representation type algebras, called Brauer configuration algebras. First introduced by Green and Schroll in [GSa] these algebras are a generalization of Brauer graph algebras, in the sense that every Brauer graph algebra is a Brauer configuration algebra. Brauer graph algebras appeared first in the mathematical literature in the more basic form of Brauer tree algebra in [Jan69]. The first general definition of a Brauer graph algebra was given by Donovan and Freislich in [DF78], where they developed a classification of the indecomposable representations of a Brauer graph algebra. The definition of a Brauer graph algebra is heavily based on combinatorial data for graphs. Underlying every Brauer graph algebra there is a finite graph with a cyclic orientation of the edges at every vertex and a multiplicity function, and these combinatorial data encode much of the representation theory of Brauer graph algebras. For example in [GSST], it is shown that the property of the Yoneda algebra of a Brauer graph algebra being finitely generated in degrees 0, 1 and 2 rely entirely on the property that its associated Brauer graph does not have both truncated and nontruncated edges; other properties about koszulness of a Brauer graph algebra also rely on the edges of the associated Brauer graph and its multiplicity function. Then, an important part of the homology and cohomology theory of a Brauer graph algebra can be studied by looking at its associated Brauer graph and the rest of its combinatorial data. So, just as the Brauer graph encodes the representation theory of Brauer graph algebras [Sch], the expectation is that the Brauer configuration will encode the representation theory of Brauer configuration algebras. As first achievement in this plan, it is shown that the Brauer configuration yields the Loewy structure of the indecomposable projective modules of a Brauer configuration algebra and that the dimension of the algebra can be directly read off the Brauer configuration [GSa, Proposition 3.13].

In the sense that a Brauer graph algebra is defined by a Brauer graph, a Brauer configuration algebra is defined by a Brauer configuration. In general terms, a Brauer configuration is a generalized idea of a Brauer graph. We can speak about the graph of a Brauer graph as an object formed by edges, and these edges in turn are formed by sets having always just two vertices. In a Brauer configuration we also have a "graph" which is formed by some kind of "edges" that we call polygons, and these polygons may have more than two vertices. But, 
as in the Brauer graph, the idea of the cyclic orientation over the set of the edges at every vertex and that of the multiplicity function, remains the same for Brauer configurations. That is, in a Brauer configuration we also have a cyclic orientation over the polygons at every vertex and a multiplicity funtion defined over the vertices.

In [Sch], and also in [Rog], it is proved that a symmetric special biserial algebra always coincide with a Brauer graph algebra. In an analogous way it is proved in [GS16, Theorem 4.1] that a symmetric special multiserial algebra always coincide with a Brauer configuration algebra, and vice-versa. The definition of a multiserial algebra, which is a generalization of a biserial algebra, was introduced first in [HHJW84], and as the special multiserial algebras are multiserial algebras, in particular any Brauer configuration algebra is also multiserial. There is an alternative way to define Brauer configuration algebras by using the concept of defining pairs. The idea behind this definition is to consider a multiplicity function with integer value over the set of all simple cycles of a quiver that satisfy five properties [GS17, Section 3]. By using this way to define Brauer configuration algebras it is possible to make relations between almost gentle algebras and Brauer configuration algebras [GSb].

We start the presentation of this work by giving in the first two sections of the first chapter all necessary basic definitions, notions and propositions about finite dimensional algebras, quivers and quotients of paht algebras. In Section 1.3 we start the exposition of Brauer configurations with a couple of examples before giving the formal definition of what is a Brauer configuration. After this, we give the instructions to form the induced quiver of the Brauer configuration and the definiton of the relations of type one, two and three in the path algebra of the induced quiver. Finally, in Definition 1.3.13 we give what is known as a Brauer configuration algebra. The next subsections present the definition and notation of special cycles, and also a characteristic partition of these objects in the induced quiver. In Subsections 1.3.4 and 1.3.5 we present the basic properties that are satisfied by any Brauer configuration algebra. All these results can be found with more accuracy in [GSa]. First chapter finishes with a section about induced boundary maps. In this section we present a light exposition about minimal bi-projective resolutions, and we show the specific segment of a minimal bi-projective resolution that we use as the main tool to calculate the center of a Brauer configuration algebra. All technical details are taken from [BM98].

The first section of Chapter two is devoted to determining a vector basis for the space of all oriented cycles through a vertex of a Brauer configuration algebra. From the definition of special cycles associated to a vertex of the configuration, we see that these special cycles are part of this vector basis. However, when the vertex in the quiver is associated to a selffolded polygon of the configuration, we observe that not only the basis is formed by special cycles but also by another type of cycle that depends on the number of times that a vertex is repeated in the polygon. By using a graphical representation, which consists in putting consecutively and cyclic manner all the arrows associated to one vertex of the configuration, we'll be able to recognize what type of cycles are missing in the desired basis, and here in this part we define the non-special cycles. The section finishes with the proof of Proposition 2.1.3, which gives the value of the dimension of the space of all oriented cycles through a vertex in a Brauer configuration algebra. This result is very important for computing the dimension of the center. The remaining part of the section considers a small set of characteristic elements that is contained in the center of a Brauer configuration algebra. 
In the next section of Chapter we present all the details of the computation of a vector basis for the center of a Brauer configuration algebra, using as a main tool the segment of a minimal bi-projective resolution introduced in Section 1.4, and a few examples using the obtained formula are given. 


\section{Chapter 1}

\section{Preliminaries}

In this chapter we introduce definitions and basic concepts to define one of the principal objects of our work: Brauer Configuration Algebras. But before that we will recall some definitions, examples and basic results about algebras, quivers and path algebras. Some results of this part will have a proof and others results don't. All details of what we mention in the first two sections can be found with accuracy in the excellent textbooks [ASS06], [Sch14] and [ARS97].

\section{$1.1 \quad$ Basic concepts}

Let $K$ be a field.

Definition 1.1.1. A $K$-algebra $\Lambda$ is a ring $(\Lambda,+, \cdot)$ with identity 1 such that $\Lambda$ has also a $K$-vector space structure such that

1. the addition in the vector space $\Lambda$ is the same as in the ring $\Lambda$;

2. the scalar multiplication in the vector space $\Lambda$ is compatible with the ring multiplication, i.e, for all $\lambda \in K$ and all $a, b \in \Lambda$, we have

$$
\lambda(a b)=(\lambda a) b=a(\lambda b)=(a b) \lambda .
$$

The dimension of the algebra $\Lambda$ is the dimension of the vector space $\Lambda$.

Example 1.1.2. $\quad$ 1. The ring of polynomials $K[x]$ in one indeterminate $x$ with coefficients in $K$ is an algebra whose identity is the constant polynomial 1. The scalar multiplication by $\lambda$ is simply given by multiplying each coefficient by $\lambda$.

2. The set $\mathbb{M}_{n}(K)$ of $n \times n$ matrices with coefficients in $K$ is an algebra whose identity is the identity matrix. The ring structure is given by the addition and multiplication of matrices, and the scalar multiplication is given by multiplying each entry of a matrix by the given scalar.

3. The set of all $4 \times 4$ matrices that have the form

$$
\left(\begin{array}{llll}
* & 0 & 0 & 0 \\
0 & * & 0 & 0 \\
0 & 0 & * & 0 \\
* & * & * & *
\end{array}\right)
$$


where each $*$ represents an arbitrary element of $K$, is an algebra. The identity matrix has this form and the set is closed under addition, multiplication, and scalar multiplication.

4. If $\Lambda$ and $\Lambda^{\prime}$ are algebras, the tensor product of the algebras $\Lambda$ and $\Lambda^{\prime}$ is the algebra that has underlying vector space $\Lambda \otimes_{K} \Lambda^{\prime}$ and the multiplication is defined by

$$
\left(a_{1} \otimes b_{1}\right)\left(a_{2} \otimes b_{2}\right)=a_{1} a_{2} \otimes b_{1} b_{2},
$$

where $a_{1}, a_{2} \in \Lambda$ and all $b_{1}, b_{2} \in \Lambda^{\prime}$ are basis elements. If $1_{\Lambda}$ and $1_{\Lambda^{\prime}}$ are the identities of $\Lambda$ and $\Lambda^{\prime}$ repectively, then $\Lambda \otimes_{K} \Lambda^{\prime}$ has identity $1_{\Lambda} \otimes 1_{\Lambda^{\prime}}$.

5. If $\Lambda$ is an algebra, then the opposite algebra $\Lambda^{\text {op }}$ is defined on the same underlying vector space by the multiplication rule $a b$ in $\Lambda^{\text {op }}$ is the same as $b a$ in $\Lambda$.

6. The enveloping algebra of an algebra $\Lambda$ is defined to be the tensor product of $\Lambda^{\mathrm{op}}$ and $\Lambda$, i.e, the algebra $\Lambda^{\mathrm{op}} \otimes_{K} \Lambda$.

7. Let $\Lambda$ be an algebra and $\mathcal{B}=\left\{b_{1}, \ldots, b_{r}\right\}$ be a basis for the underlying vector space $\Lambda$. If $a, a^{\prime} \in \Lambda$ then there exist scalars $\lambda_{i}, \lambda_{j}^{\prime}$ in $K$ such that $a=\lambda_{1} b_{1}+\cdots+\lambda_{r} b_{r}$ and $a^{\prime}=\lambda_{1}^{\prime} b_{1}+\cdots+\lambda_{r}^{\prime} b_{r}$. Then their product must satisfy

$$
a a^{\prime}=\left(\sum_{i=1}^{r} \lambda_{i} b_{i}\right)\left(\sum_{j=1}^{r} \lambda_{j}^{\prime} b_{j}\right)=\sum_{i=1}^{r}\left(\sum_{j=1}^{r} \lambda_{i} \lambda_{j}^{\prime} b_{i} b_{j}\right)
$$

which means that, if we specify how to multiply any two basis elements $b_{i}, b_{j}$, and also ensure that this specification is associative over the elements of the basis, then the multiplication in the algebra is completely determined. We say that we extend the multiplication from the basis to the whole algebra by linearity.

Throughout this thesis when we say that $M$ is a $\Lambda$-module we are saying right $\Lambda$-module, unless something different is explicitly mentioned.

Definition 1.1.3. Let $\Lambda$ be an algebra.

1. An element $e \in \Lambda$ is called idempotent if $e^{2}=e$.

2. If $e_{1}, e_{2} \in \Lambda$ are idempotents such that $e_{1} e_{2}=e_{2} e_{1}=0$, we say that $e_{1}$ and $e_{2}$ are orthogonal idempotents.

3. An idempotent $e \in \Lambda$ is said to be primitive if $e$ cannot be written as a sum $e=e_{1}+e_{2}$, where $e_{1}$ and $e_{2}$ are nonzero orthogonal idempotents of $\Lambda$.

4. If $e \in \Lambda$ is an idempotent such that

$$
e a=a e, \text { for all } a \in \Lambda,
$$

then we call it a central idempotent.

5. Let $\left\{e_{1}, \ldots, e_{s}\right\}$ be a subset of $\Lambda$ such that $e_{i}$ is idempotent for all $1 \leq i \leq s$, and $e_{i} e_{j}=0$ for $i \neq j$. If $\Lambda$ has identity 1 and

$$
\sum_{i=1}^{s} e_{i}=1
$$


then we say that $\left\{e_{1}, \ldots, e_{s}\right\}$ is a complete set of orthogonal idempotents for $\Lambda$.

We state the following proposition whitout a proof. We refer the reader to [ASS06, Corollary 4.7] for details of it.

Proposition 1.1.4. Let $\Lambda$ be a $K$-algebra and $e \in \Lambda$ be an idempotent. Then $e$ is primitive if and only if the $K$-algebra $e \Lambda e$ has only two idempotents, 0 and $e$.

We say that an algebra $\Lambda$ is connected (or indecomposable) if $\Lambda$ is not a direct product of two algebras, or equivalently, if 0 and 1 are the only central idempotents of $\Lambda$.

The following lemma reduces the connectedness of an algebra to a partition of a complete set of primitive orthogonal idempotents for this algebra. It will be helpful to characterise connected path algebras and connected quotients of path algebras, later in Section 1.2.

Lemma 1.1.5. Let $\Lambda$ be a $K$-algebra with identity and suppose that $\left\{e_{1}, \ldots, e_{n}\right\}$ is a complete set of primitive orthogonal idempotents. Then $\Lambda$ is connected if an only if there does not exist a nontrivial partition $A \cup \dot{\cup}$ of the set $\{1, \ldots, n\}$ such that

$$
i \in A \text { and } j \in B \Longrightarrow e_{i} \Lambda e_{j}=0=e_{j} \Lambda e_{i} .
$$

Proof. Let $A \cup \dot{\cup} B$ be a nontrivial partition of $\{1, \ldots, n\}$, and let $c$ be the element of $\Lambda$ given by

$$
c=\sum_{i \in A} e_{i}
$$

Because the partition is nontrivial, $c \neq 0$ and $c \neq 1$. The element $e_{i}$ is orthogonal and idempotent, for each $i \in A$, then $c$ is an idempotent. Besides,

- $c e_{i}=e_{i} c=e_{i}$, for every $i \in A$;

- $c e_{j}=e_{j} c=0$, for every $j \in B$.

Let $a$ be an arbitrary element of $\Lambda$. By hypothesis, $e_{i} a e_{j}=0=e_{j} a e_{i}$ whenever $i \in A$ and $j \in B$. Then

$$
\begin{aligned}
c a & =\left(\sum_{i \in A} e_{i}\right) a 1 \\
& =\left(\sum_{i \in A} e_{i} a\right)\left(\sum_{k \in A} e_{k}+\sum_{j \in B} e_{j}\right) \\
& =\sum_{i \in A}\left(\sum_{k \in A} e_{i} a e_{k}\right) \\
a c & =1 a\left(\sum_{k \in A} e_{k}\right) \\
& =\left(\sum_{i \in A} e_{i}+\sum_{j \in B} e_{j}\right)\left(\sum_{k \in A} a e_{k}\right) \\
& =\sum_{i \in A}\left(\sum_{k \in A} e_{i} a e_{k}\right) .
\end{aligned}
$$


We see that (1.1) and (1.2) are equal, hence $c$ is a central idempotent, and $\Lambda=c \Lambda \times(1-c) \Lambda$ is a nontrivial product decomposition of $\Lambda$.

Conversely, suppose that $\Lambda$ is not connected, and let $c$ be a nontrivial central idempotent of $\Lambda$, i.e, $c \neq 0,1$. Then we have

$$
c=1 c 1=\left(\sum_{i=1}^{n} e_{i}\right) c\left(\sum_{j=1}^{n} e_{j}\right)=\sum_{i=1}^{n}\left(\sum_{j=1}^{n} e_{i} c e_{j}\right)=\sum_{i=1}^{n} e_{i} c e_{i} .
$$

Set $c_{i}=e_{i} c e_{i}$. It is clear that $c_{i} \in e_{i} \Lambda e_{i}$. Because $c$ is a central idempotent it follows that $c_{i}$ is an idempotent of $e_{i} \Lambda e_{i}$. Each $e_{i}$ is primitive, then $c_{i}=0$ or $c_{i}=e_{i}$ by Proposition 1.1.4. Let $A$ and $B$ be sets defined by

$$
A=\left\{i \mid c_{i}=0\right\} \text { and } B=\left\{j \mid c_{j}=e_{j}\right\}
$$

Because $c \neq 1,0$, this pair of sets form indeed a nontrivial partition of $\{1, \ldots, n\}$. Now, if $i \in A$ and $j \in B$ we obtain

$$
\begin{gathered}
0=c_{i}=e_{i} c e_{i}=e_{i} c=c e_{i}, \\
e_{j}=c_{j}=e_{j} c e_{j}=e_{j} c=c e_{j} .
\end{gathered}
$$

Therefore, if $i \in A$ and $j \in B$, we have $e_{i} \Lambda e_{j}=e_{i} \Lambda c e_{j}=e_{i} c \Lambda e_{j}=0$ and $e_{j} \Lambda e_{i}=e_{j} c \Lambda e_{i}=$ $e_{j} \Lambda c e_{i}=0$.

The following lemma will be useful in Section 1.4.

Lemma 1.1.6. Let $\Lambda$ be a $K$-algebra , $e \in \Lambda$ be an idempotent, and $M$ be a righ $\Lambda$-module. Then

$$
M e \cong \operatorname{Hom}_{\Lambda}(e \Lambda, M)
$$

as right $e \Lambda e$-modules.

Proof. Let $\phi_{1}: M e \rightarrow \operatorname{Hom}_{\Lambda}(e \Lambda, M)$ and $\phi_{2}: \operatorname{Hom}_{\Lambda}(e \Lambda, M) \rightarrow M e$ be the maps defined by

$$
\begin{aligned}
\phi_{1}(x e) & : \quad e a \mapsto x e a \\
\phi_{2} & : \varphi \mapsto \varphi(e),
\end{aligned}
$$

respectively. It is easy to check that both maps are $e \Lambda e$-homomorphism, and $\phi_{1}$ and $\phi_{2}$ are mutually inverse.

Recall that $M$ is a $\Lambda$-bimodule if $M$ is a right $\Lambda$-module and a left $\Lambda$-module and satisfies

$$
(a x) b=a(x b) \text {, for all } x \in M \text { and } a, b \in \Lambda .
$$

Because of this associativity we just write $a x b$ instead of $a(x b)$ or $(a x) b$. Also recall the enveloping algebra of $\Lambda$ as the $K$-algebra given by $\Lambda^{e}=\Lambda^{\mathrm{op}} \otimes_{K} \Lambda$. The following proposition is easy to prove. We left the proof to the reader.

Proposition 1.1.7. Let $\Lambda$ be a $K$-algebra. If $M$ is a $\Lambda$-bimodule, then $M$ is a right $\Lambda^{e}$ module with the multiplication defined by

$$
x(a \otimes b)=a x b, \text { for all } x \in M \text { and } a, b \in \Lambda .
$$

The $K$-algebra $\Lambda$ is naturally a $\Lambda$-bimodule, then we can see $\Lambda$ as right $\Lambda^{e}$-module. It is well known that categories of $\Lambda$-bimodules and right $\Lambda^{e}$-modules are isomorphic. We will 
freely switch back and forth between $\Lambda$-bimodules and right $\Lambda^{e}$-modules, choosing the most convenient formulation or statement.

\subsection{Quivers and path algebras}

First of all, we need a formal definition of finite quivers.

Definition 1.2.1. A finite quiver is a quadruple $\mathcal{Q}=\left(\mathcal{Q}_{0}, \mathcal{Q}_{1}, s, t\right)$ consisting of

- $\mathcal{Q}_{0}$ a finite set of vertices;

- $\mathcal{Q}_{1}$ a finite set of arrows;

- $s: \mathcal{Q}_{1} \rightarrow \mathcal{Q}_{0}$ a map from arrows to vertices, mapping an arrow to its starting point;

- $t: \mathcal{Q}_{1} \rightarrow \mathcal{Q}_{0}$ a map from arrows to vertices, mapping an arrow to its terminal point.

Remark: The general definition of quivers does not require $\mathcal{Q}_{0}$ and $\mathcal{Q}_{1}$ finite. In this thesis all quivers are finite and we call them simply quivers.

An arrow $a \in \mathcal{Q}_{1}$ with starting point $v=s(a)$ and terminal point $w=t(a)$ is usually represented by $a: v \rightarrow w$ or by

$$
v \stackrel{a}{\longrightarrow} w .
$$

A quiver $\mathcal{Q}=\left(\mathcal{Q}_{0}, \mathcal{Q}_{1}, s, t\right)$ is also usually denoted briefly $\mathcal{Q}=\left(\mathcal{Q}_{0}, \mathcal{Q}_{1}\right)$ or even simply by $\mathcal{Q}$. So, a quiver is nothing but an oriented graph without any restriction on the number of arrows between two vertices, the existence of loops or oriented cycles. When a quiver is draw, we agree to represent each vertex by either a small alphabet letter with or without subindex, and each arrow will be pointing towards its target. Arrows will be labeled with the few first of the alphabet letter with or without subindex, and with or without superindex

Example 1.2.2. The following quiver is given by $\mathcal{Q}_{0}=\left\{v_{1}, v_{2}, v_{3}, v_{4}\right\}, \mathcal{Q}_{1}=\left\{a_{1}, a_{2}, a_{3}, a_{4}, a_{5}, a_{6}, a_{7}\right\}$ and

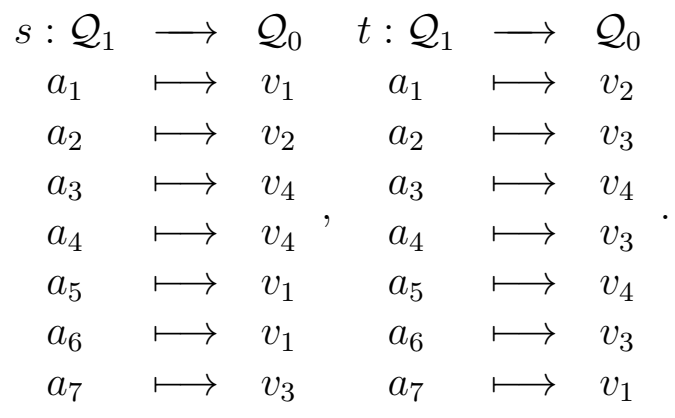

We can draw the quiver as

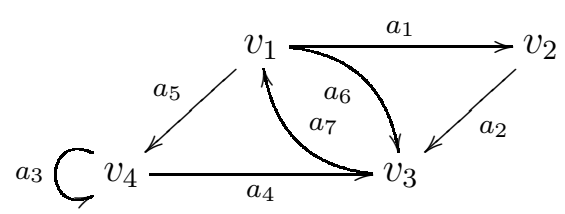

A subquiver of a quiver $\mathcal{Q}=\left(\mathcal{Q}_{0}, \mathcal{Q}_{1}, s, t\right)$ is a quiver $\mathcal{Q}^{\prime}=\left(\mathcal{Q}_{0}^{\prime}, \mathcal{Q}_{1}^{\prime}, s^{\prime}, t^{\prime}\right)$ such that

- $\mathcal{Q}_{0}^{\prime} \subset \mathcal{Q}_{0}$ and $\mathcal{Q}_{1}^{\prime} \subset \mathcal{Q}_{1}$;

- $s^{\prime}=\left.s\right|_{\mathcal{Q}_{1}^{\prime}}$ and $t^{\prime}=\left.t\right|_{\mathcal{Q}_{1}^{\prime}}$. 
Such a subquiver is called full if $\mathcal{Q}_{1}^{\prime}$ coincides with the set of all those arrows in $\mathcal{Q}_{1}$ whose source and target both belong to $\mathcal{Q}_{0}^{\prime}$, i.e,

$$
\mathcal{Q}_{1}^{\prime}=\left\{a \in \mathcal{Q}_{1} \mid s(a) \in \mathcal{Q}_{0}^{\prime} \text { and } t(a) \in \mathcal{Q}_{0}^{\prime}\right\} .
$$

Example 1.2.3. Let $\mathcal{Q}$ and $\mathcal{Q}^{\prime}$ be the quivers given by
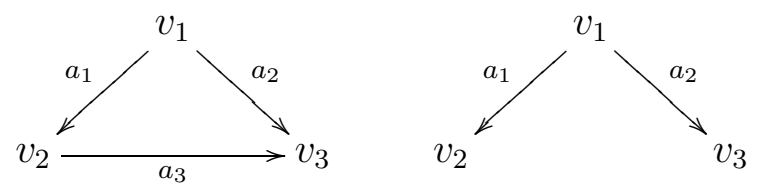

respectively. We see that $\mathcal{Q}^{\prime}$ is a subquiver of $\mathcal{Q}$ where $s\left(a_{3}\right) \in \mathcal{Q}_{0}^{\prime}$ and $t\left(a_{3}\right) \in \mathcal{Q}_{0}^{\prime}$ but $a_{3} \notin \mathcal{Q}_{1}^{\prime}$. Hence $Q^{\prime}$ cannot be a full subquiver of $\mathcal{Q}$.

Let $\mathcal{Q}$ be a quiver. A path of $\mathcal{Q}$ of length $l>1$ is a sequence $a_{1}, a_{2} \ldots, a_{l}$ of arrows of $\mathcal{Q}$ such that $t\left(a_{i}\right)=s\left(a_{i+1}\right)$, for all $1 \leq i \leq l-1$. Such a path is denoted by $a_{1} a_{2} \cdots a_{l}$. If $s\left(a_{1}\right)=v$ and $t\left(a_{l}\right)=w$ we set $p=a_{1} a_{2} \cdots a_{l}$ and say that the path $p$ is from $v$ to $w$ and define $s(p)=v$ and $t(p)=w$. The length of $p$ is usually denoted by $\ell(p)=l$. For every $v \in \mathcal{Q}_{0}$ we agree to associate a path of length $\ell=0$, called the trivial path at $v$. We denote such a path also by $v$. All elements $a \in \mathcal{Q}_{1}$ are defined as a path such that $\ell(a)=1$.

Let $p$ and $q$ be two paths in the quiver $\mathcal{Q}$, and suppose that $p=a_{1} a_{2} \cdots a_{r}$ and $q=$ $b_{1} b_{2} \cdots b_{s}$ with $r \geq 1$ and $s \geq 1$. If $t(p)=s(q)$ we denote by $p \cdot q$ the concatenation of the two paths as the path of $\mathcal{Q}$ given by

$$
p \cdot q:=a_{1} a_{2} \cdots a_{r} b_{1} b_{2} \cdots b_{s}
$$

If $v, w \in \mathcal{Q}_{0}$ are vertices such that $s(p)=v$ and $t(p)=w$ we define

$$
v \cdot p=p \cdot w:=p
$$

Definition 1.2.4. Let $\mathcal{Q}$ be a quiver. The path algebra $K \mathcal{Q}$ of $\mathcal{Q}$ is the $K$-algebra whose underlying $K$-vector space has as its basis the set of all paths in $\mathcal{Q}$ with length $\ell \geq 0$, and with multiplication of two basis elements $p$ and $q$ given by

$$
p q=\left\{\begin{array}{cl}
p \cdot q, & \text { if } t(p)=s(q) \\
0, & \text { otherwise }
\end{array}\right.
$$

Example 1.2.5. (1) Let $\mathcal{Q}$ be the quiver with one vertex and one arrow given by

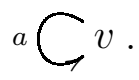

A $K$-basis of $K \mathcal{Q}$ is $\left\{v, a, a^{2}, a^{3}, a^{4}, \ldots\right\}$. If we define $a^{0}=v$ and the multiplication by $a^{k} a^{l}=a^{k+l}$ for all $k, l \geq 0$, then is not difficult to prove that $K \mathcal{Q}$ is isomorphic to the polynomial algebra $K[x]$ in one indeterminate $x$. In fact, such an isomorphism is induced by the $K$-linear map $K \mathcal{Q} \rightarrow K[x]$ defined as

$$
v \mapsto 1, a \mapsto x .
$$

(2) Let $\mathcal{Q}$ be the quiver

$$
{ }^{a}{ }^{v}{ }^{b}
$$

A $K$-basis of $K \mathcal{Q}$ is the set of all words on $\{a, b\}$ with the empty word equal to $v$ the 
identity of $K \mathcal{Q}$. The multiplication of basis vectors reduces to the multiplication in the free monoid over $\{a, b\}$. If $K\langle x, y\rangle$ is the free associative algebra in two noncommuting indeterminates, then the $K$-linear map $K \mathcal{Q} \rightarrow K\langle x, y\rangle$ induced by

$$
v \mapsto 1, a \mapsto x, b \mapsto y
$$

is an isomorphism of algebras. In general, if we take a quiver formed by only one vertex and $n$ arrows then the associated path algebra is isomorphic to the free associative algebra in $n$ noncommuting indeterminates.

A path $p$ of a quiver $\mathcal{Q}$ is called a cycle if satisfies that $s(p)=t(p)$ and $\ell(p) \geq 1$. If $p$ is a cycle such that $\ell(p)=1$ we call it a loop. If $p$ is a cycle and $s(p)=t(p)=v$ we say that $p$ is a cycle through $v$ or simply a cycle at $v$. The quiver $\mathcal{Q}$ is called acyclic if it contains no cycles.

Lemma 1.2.6. Let $\mathcal{Q}$ be a quiver and $K \mathcal{Q}$ be its path algebra. Then

(1) the sum of all trivial paths of $\mathcal{Q}$ coincides with the identity of $K \mathcal{Q}$, i.e,

$$
\sum_{v \in \mathcal{Q}_{0}} v=1_{K \mathcal{Q}}
$$

(2) the set $\left\{v \mid v \in \mathcal{Q}_{0}\right\}$ of all the stationary paths is a complete set of primitive orthogonal idempotents for $K \mathcal{Q}$;

(3) $K \mathcal{Q}$ is finite dimensional if and only if $\mathcal{Q}$ is acyclic.

Proof. 1. By the definition of multiplication is easy to check that

$$
\left(\sum_{v \in \mathcal{Q}_{0}} v\right) p=p\left(\sum_{v \in \mathcal{Q}_{0}} v\right),
$$

for any path $p$ of $\mathcal{Q}$. Then it follows the affirmation.

2. By (1) and the definition of multiplication, there remains that each $v$ in $\mathcal{Q}$ is primitive. By Proposition 1.1.4 it is enough to show that the algebra $v(K \mathcal{Q}) v$ only has two idempotents, 0 and $v$. Indeed, suppose that $e$ is an idempotent of $v(K \mathcal{Q}) v$ then $e$ can be written in the form

$$
e=\alpha v+C
$$

where $\alpha \in K$ and $C$ is a linear combination of cycles throuhg $v$ of length $\geq 1$. We have that $e^{2}=e$, then the equality

$$
0=e^{2}-e=\left(\alpha^{2}-\alpha\right) v+(2 \alpha-1) C+C^{2}
$$

implies that $\alpha^{2}=\alpha$. In either case $\alpha=0$ or $\alpha=1$ we obtain that $C=0$. Then if $\alpha=0$ then $e=0$; if $\alpha=1$ then $e=v$.

3. If $p$ is a cycle in $\mathcal{Q}$ then the set $\left\{p^{t} \mid t \geq 1\right\}$ is contained in the $K$-basis of $K \mathcal{Q}$. So the dimension of $K \mathcal{Q}$ is not finite. Conversely, if $\mathcal{Q}$ is acyclic, it contains only finitely many paths so $K \mathcal{Q}$ is finite dimensional. 
Lemma 1.2.7. Let $\mathcal{Q}$ be a quiver. The path algebra $K \mathcal{Q}$ is connected if and only if $\mathcal{Q}$ is a connected quiver.

Proof. Suppose that $\mathcal{Q}$ is not a connected quiver, and let $\mathcal{Q}^{\prime}$ be a connected component of $\mathcal{Q}$. Let $\mathcal{Q}^{\prime \prime}$ denote the subquiver of $\mathcal{Q}$ given by $\mathcal{Q}_{0}^{\prime \prime}=\mathcal{Q}_{0} \backslash \mathcal{Q}_{0}^{\prime}$ and $\mathcal{Q}_{1}^{\prime \prime}=\left\{a \in \mathcal{Q}_{1} \mid s(a) \in \mathcal{Q}_{0}^{\prime \prime}\right.$ and $\left.t(a) \in \mathcal{Q}_{0}^{\prime \prime}\right\}$. It is clear that $\mathcal{Q}^{\prime \prime}$ is a full subquiver of $\mathcal{Q}$. Because $\mathcal{Q}$ is not connected neither $\mathcal{Q}^{\prime}$ nor $\mathcal{Q}^{\prime \prime}$ is empty. Let $p$ be an arbitrary path in $\mathcal{Q}$, and let $v_{1} \in \mathcal{Q}_{0}^{\prime}$ and $v_{2} \in \mathcal{Q}_{0}^{\prime \prime}$. So $p$ is entirely contained in either $\mathcal{Q}^{\prime}$ or $\mathcal{Q}^{\prime \prime}$. In the former case, we have $p v_{2}=0$ and hence $v_{1} p v_{2}=0$. In the latter case, we have $v_{1} p=0$ and hence $v_{1} p v_{2}=0$. This shows that $v_{1}(K \mathcal{Q}) v_{2}=0$. Similarly, $v_{2}(K \mathcal{Q}) v_{1}=0$. By Lemma 1.1.5 it follows that $K \mathcal{Q}$ is not connected.

Conversely, suppose now that $\mathcal{Q}$ is connected but $K \mathcal{Q}$ is not. By Lemma 1.1.5 there exists a disjoint union partition $\mathcal{Q}_{0}=\mathcal{Q}_{0}^{\prime} \cup \mathcal{Q}_{0}^{\prime \prime}$ such that, if $v \in \mathcal{Q}_{0}^{\prime}$ and $w \in \mathcal{Q}_{0}^{\prime \prime}$ then $v(K \mathcal{Q}) w=0=w(K \mathcal{Q}) v$. Because $\mathcal{Q}$ is connected there exists an arrow $a \in \mathcal{Q}_{1}$ and vertices $v_{1} \in \mathcal{Q}_{0}^{\prime}$ and $v_{2} \in \mathcal{Q}_{0}^{\prime \prime}$ such that $s(a)=v_{1}$ and $t(a)=v_{2}$. But this implies that

$$
a=v_{1} a v_{2} \in v_{1}(K \mathcal{Q}) v_{2}=0,
$$

a contradiction. This completes the proof.

Definition 1.2.8. Let $\mathcal{Q}$ be a quiver and let $J$ denote the ideal of $K \mathcal{Q}$ generated by all the arrows in $\mathcal{Q}$. A two-sided ideal $I$ of $K \mathcal{Q}$ is said to be admissible if there exists an integer $n \geq 2$ such that

$$
J^{n} \subset I \subset J^{2}
$$

For $x \in K \mathcal{Q}$ we denote by $\bar{x}$ the class $x+I$ in $K \mathcal{Q} / I$. We state the following lemma without proof.

Lemma 1.2.9. Let $\mathcal{Q}$ be a quiver and $I$ be an admissible ideal of $K \mathcal{Q}$.

(1) The set $\left\{\bar{v} \mid v \in \mathcal{Q}_{0}\right\}$ is a complete set of primitive orthogonal idempotents of the algebra $K \mathcal{Q} / I$.

(2) $K \mathcal{Q}$ is connected if and only if $\mathcal{Q}$ is a connected quiver.

(3) $K \mathcal{Q} / I$ is finite dimensional.

\subsection{Brauer configuration algebras}

Brauer configuration algebras arise as a natural generalization of Brauer graph algebras, and as with Brauer graph algebras, Brauer configuration algebras are defined by combinatorial data, call it Brauer configuration. Introduced first by E. Green and S. Schroll in [GSa] these algebras are finite dimensional symmetric and mostly of wild representation type. The fact that a Brauer configuration algebra is a generalization of Brauer graph algebra is because every Brauer graph is a Brauer configuration and every Brauer graph algebra is a Brauer configuration algebra. The Brauer graph encodes the representation theory of a Brauer graph algebra, and the expectation is that the Brauer configuration will encode the representation theory of Brauer configuration algebra.

\subsubsection{Brauer configuration}

Let $\Gamma=\left(\Gamma_{0}, \Gamma_{1}\right)$ where $\Gamma_{0}$ is a finite set of elements that we call vertices of $\Gamma$ and $\Gamma_{1}$ is a finite collection of labeled finite sets of vertices where repetitions are allowed. Another way 
to say it is that $\Gamma_{1}$ is a finite collection of finite multisets of vertices. We call each element of $\Gamma_{1}$ a polygon.

Example 1.3.1. As a start we provide two simple examples that we are going to use troughout the chapter to clarify both definitions and concepts.

(a) $\Gamma=\left(\Gamma_{0}, \Gamma_{1}\right)$ is the tuple given by $\Gamma_{0}=\{1,2,3\}$ and $\Gamma_{1}=\left\{V_{1}, V_{2}, V_{3}, V_{4}\right\}$ where

$$
\begin{array}{ll}
V_{1}=\Gamma_{0} & V_{2}=\{1,2\} \\
V_{3}=\{2,3\} & V_{4}=\{3,1\}
\end{array}
$$

(b) $\Gamma^{\prime}=\left(\Gamma_{0}^{\prime}, \Gamma_{1}^{\prime}\right)$ is the tuple given by $\Gamma_{0}^{\prime}=\{\alpha, \beta\}$ and $\Gamma_{1}^{\prime}=\{V, W\}$ where

$$
V=\{\alpha, \alpha, \alpha, \beta\}, W=\{\beta, \beta, \beta, \alpha\} .
$$

In this particular configuration we can see that in both polygons $V$ and $W$ there are repetitions of vertices.

For $V \in \Gamma_{1}$ we call the elements of $V$ the vertices of $V$. If $\alpha$ is a vertex in $\Gamma_{0}$, define the value occ $(\alpha, V)$ as the number of times that $\alpha$ occurs as a vertex in $V$ and the valence of $\alpha$ as the nonnegative integer

$$
\operatorname{val}(\alpha):=\sum_{V \in \Gamma_{1}} \operatorname{occ}(\alpha, V)
$$

From the previous example we see that the tuple in (a) satisfies

$$
\begin{array}{ll}
\operatorname{occ}\left(1, V_{1}\right)=\operatorname{occ}\left(1, V_{2}\right)=\operatorname{occ}\left(1, V_{4}\right)=1, & \operatorname{occ}\left(1, V_{3}\right)=0, \\
\operatorname{occ}\left(2, V_{1}\right)=\operatorname{occ}\left(2, V_{2}\right)=\operatorname{occ}\left(2, V_{3}\right)=1, & \operatorname{occ}\left(2, V_{4}\right)=0, \\
\operatorname{occ}\left(3, V_{1}\right)=\operatorname{occ}\left(3, V_{3}\right)=\operatorname{occ}\left(3, V_{4}\right)=1, & \operatorname{occ}\left(3, V_{2}\right)=0 .
\end{array}
$$

So we have that $\operatorname{val}(i)=3$, for $i=1,2,3$. While in the tuple in (b) we have $\operatorname{occ}(\alpha, V)=3=$ $\operatorname{occ}(\beta, W), \operatorname{occ}(\beta, V)=1=\operatorname{occ}(\alpha, W)$ and $\operatorname{val}(\alpha)=\operatorname{val}(\beta)=4$.

To define what exactly a Brauer configuration is we need two more data. One of these data is a function $\mu: \Gamma_{0} \rightarrow \mathbb{Z}^{>0}$ that we will call a multiplicity function, where $\mathbb{Z}^{>0}$ is the set of the positive integers.

Example 1.3.2. For the tuple $\Gamma$ in (a) in Example 1.3 .1 we may choose $\mu: \Gamma_{0} \rightarrow \mathbb{Z}^{>0}$ defined by

$$
\mu(i)=N, \forall i \in \Gamma_{0},
$$

where $N>1$ is an integer. And for the tuple $\Gamma^{\prime}$ in (b) we define $\mu: \Gamma_{0}^{\prime} \rightarrow \mathbb{Z}^{>0}$ as $\mu(\alpha)=$ $\mu(\beta)=1$.

The other data is what we call an orientation $\mathfrak{o}$ of $\Gamma$. An orientation $\mathfrak{o}$ of $\Gamma$ is a choice, for each vertex $\alpha \in \Gamma_{0}$, of a cyclic ordering of the polygons in which $\alpha$ occurs as a vertex, including repetitions. Adding more accuracy, for each $\alpha \in \Gamma_{0}$, let $V_{1}, \ldots, V_{t}$ be the list of polygons in which $\alpha$ occurs as a vertex, with a polygon $V \operatorname{occurring} \operatorname{occ}(\alpha, V)$ times in the list, that is $V$ occurs the number of times $\alpha$ occurs as a vertex in $V$. The cyclic order at vertex $\alpha$ is obtained by linearly ordering the list, say $V_{i_{1}}<\cdots<V_{i_{t}}$ and by adding $V_{i_{t}}<V_{i_{1}}$. We observe that any cyclic permutation of a chosen cyclic ordering at vertex $\alpha$ can represent the same ordering. That is, if $V_{1}<\cdots<V_{t}$ is the chosen cyclic ordering at vertex $\alpha$, so it is a cyclic permutation such as $V_{2}<V_{3}<\cdots<V_{t}<V_{1}$ or $V_{3}<V_{4}<\cdots<V_{t}<V_{1}<V_{2}$. 
Example 1.3.3. In the tuple $\Gamma$ of Example 1.3.1(a) we have that the list of polygons ocurring in any vertex has three polygons. The list of polygons occurring at vertex 1 is $V_{1}, V_{2}, V_{4}$; at vertex 2 the list is $V_{1}, V_{2}, V_{3}$ and at vertex 3 the list is $V_{1}, V_{3}, V_{4}$. For the tuple $\Gamma^{\prime}$ in (b) of Example 1.3.1 we have that, putting repetitions, the list of polygons ocurring at $\alpha$ is $V, V, V, W$, and for the vertex $\beta$ the list is $W, W, W, V$. Now, an orientation for $\Gamma$ is given by

$$
\begin{array}{lll}
\text { at vertex } 1: & V_{1}<V_{4}<V_{2}, \\
\text { at vertex } 2: & V_{1}<V_{2}<V_{3}, \\
\text { at vertex } 3: & V_{1}<V_{3}<V_{4} .
\end{array}
$$

Observe that for each vertex in $\Gamma_{0}$ there are only 2 choices for cyclic ordering. So we could choose a different order for each vertex. In the tuple $\Gamma^{\prime}$ we have only one possible cyclic ordering for any vertex in $\Gamma_{0}^{\prime}$.

$$
\begin{aligned}
& \text { at vertex } \alpha: V<V<V<W \text {, } \\
& \text { at vertex } \beta \text { : } W<W<W<V \text {. }
\end{aligned}
$$

Let $\mathfrak{o}\left(\Gamma^{\prime}\right)$ denote the orientation for the configuration $\Gamma^{\prime}$, and $\mathfrak{o}(\Gamma)$ denote the orientation for the configuration $\Gamma$.

Now we give the first important definition of this section, the prime object of our work.

Definition 1.3.4. A Brauer configuration is a tuple $\Gamma=\left(\Gamma_{0}, \Gamma_{1}, \mu, \mathfrak{o}\right)$, where $\Gamma_{0}$ is a set of vertices, $\Gamma_{1}$ a set of polygons, $\mu$ is a multiplicity function, and $\mathfrak{o}$ is an orientation for $\Gamma$, such that the following conditions hold

C1. Every vertex in $\Gamma_{0}$ is a vertex in at least one polygon in $\Gamma_{1}$.

C2. Every polygon in $\Gamma_{1}$ has at least two vertices.

C3. Every polygon in $\Gamma_{1}$ has at least one vertex $\alpha$ such that $\operatorname{val}(\alpha) \mu(\alpha)>1$.

It could happen that two different polygons in $\Gamma_{1}$ both have the same set of vertices. If $V$ and $V^{\prime}$ are polygons of this kind we distinguish between the two using their labels $V$ and $V^{\prime}$. For the particular tuple $(\{1,2\},\{V=\{1,2\}\}, \mu, \mathfrak{o})$ where $\mu(1)=\mu(2)=1$ we see that does not satisfy the condition C3 of Definition 1.3.4, and hence is not a Brauer configuration. However, by convention the induced algebra of this tuple is nevertheless considered to be a Brauer graph (see Definition 1.3.17) algebra which is isomorphic to the truncated polynomial algebra $K[x] /\left(x^{2}\right)$.

Definition 1.3.5. Let $\Gamma=\left(\Gamma_{0}, \Gamma_{1}, \mu, \mathfrak{o}\right)$ be a Brauer configuration. We say that $\Gamma$ is disconnected if there are two Brauer configurations $\Gamma^{\prime}=\left(\Gamma_{0}^{\prime}, \Gamma_{1}^{\prime}, \mu^{\prime}, \mathfrak{o}^{\prime}\right)$ and $\Gamma^{\prime \prime}=\left(\Gamma_{0}^{\prime \prime}, \Gamma_{1}^{\prime \prime}, \mu^{\prime \prime}, \mathfrak{o}^{\prime \prime}\right)$ such that

(1) $\left\{\Gamma_{0}^{\prime}, \Gamma_{0}^{\prime \prime}\right\}$ is a partition of $\Gamma_{0}$.

(2) $\left\{\Gamma_{1}^{\prime}, \Gamma_{1}^{\prime \prime}\right\}$ is a partition of $\Gamma_{1}$.

(3) For every polygon $V \in \Gamma_{1}$ the vertices of $V$ are either all in $\Gamma_{0}^{\prime}$ or are all in $\Gamma_{0}^{\prime \prime}$.

(4) $\mu^{\prime}$ (resp. $\left.\mu^{\prime \prime}\right)$ is a restriction of $\mu$ to $\Gamma_{0}^{\prime}$ (resp. $\left.\Gamma_{0}^{\prime \prime}\right)$.

(5) The orientations $\mathfrak{o}^{\prime}$ and $\mathfrak{o}^{\prime \prime}$ are induced by $\mathfrak{o}$. 
In this case, we write $\Gamma=\Gamma^{\prime} \cup \Gamma^{\prime \prime}$. When $\Gamma$ is not disconnected we say that is connected.

Because of the finiteness of the sets $\Gamma_{0}$ and $\Gamma_{1}$ it is clear that $\Gamma$ can be uniquely written as a union of a finite collection of connected Brauer configurations. We call these connected Brauer configurations the connected components of $\Gamma$.

By the definition of a Brauer configuration we know that a polygon $V \in \Gamma_{1}$ is a multiset. Then is possible that one vertex in $V$ can appear more than once. In this case we say that the polygon $V$ is self-folded. If the number of vertices appearing in $V$ is $d$ we say that $V$ is a d-gon.

Definition 1.3.6. Let $\Gamma=\left(\Gamma_{0}, \Gamma_{1}, \mu, \mathfrak{o}\right)$ be a Brauer configuration. We say that a vertex $\alpha \in \Gamma_{0}$ is truncated if $\operatorname{val}(\alpha)=\mu(\alpha)=1$. A vertex of the configuration that is not truncated is called a nontruncated vertex.

One remark which we make is that truncated vertices, when they exist, can be "omitted" in almost all the cases where they appear. There is a reduction procedure that eliminates a truncated vertex producing a new Brauer configuration that will not affect the associated Brauer configuration algebra defined in Subsection 1.3.2, see Proposition 1.3.16.

The reduction procedure for removing a truncated vertex is defined as follows. Let $\Gamma=$ $\left(\Gamma_{0}, \Gamma_{1}, \mu, \mathfrak{o}\right)$ be a Brauer configuration and $\alpha \in \Gamma_{0}$ such that $\operatorname{val}(\alpha)=\mu(\alpha)=1$. Let $V$ be the unique polygon in $\Gamma_{1}$ such that $\alpha \in V$ and suppose that $V$ is a $d$-gon. If $d=2$ then we do nothing. If $d \geq 3$ then we define the Brauer configuration $\Gamma^{\prime}=\left(\Gamma_{0}^{\prime}, \Gamma_{1}^{\prime}, \mu^{\prime}, \mathfrak{o}^{\prime}\right)$ where

(1) $\Gamma_{0}^{\prime}=\Gamma_{0} \backslash\{\alpha\}$

(2) $V^{\prime}=V \backslash\{\alpha\}$,

(3) $\Gamma_{1}^{\prime}=\left(\Gamma_{1} \backslash\{V\}\right) \cup\left\{V^{\prime}\right\}$,

(4) $\mu^{\prime}=\left.\mu\right|_{\Gamma_{0}^{\prime}}$,

(5) $\mathfrak{o}^{\prime}$ is the orientation induced from the orientation $\mathfrak{o}$.

As we can see if $d>1$, this new Brauer configuration $\Gamma^{\prime}$ is simply the configuration $\Gamma$ without the truncated vertex $\alpha$ in the polygon $V . \Gamma$ and $\Gamma^{\prime}$ both have the same number of polygons, and only one polygon in $\Gamma^{\prime}$ has one less vertex.

If $\Gamma^{\prime}$ also has a truncated vertex in a $d^{\prime}$-gon, with $d^{\prime} \geq 3$, applying the same reduction procedure we can remove it and obtain a Brauer configuration $\Gamma^{\prime \prime}$. This $\Gamma^{\prime \prime}$ has two less vertices than $\Gamma$. Continuing in this fashion we arrive to a Brauer configuration $\Gamma^{*}$ having the property that any of its truncated vertex occurs in a 2-gon and hence no more reductions can be permormed in $\Gamma^{*}$. Let $\mathscr{T}_{\Gamma}$ be the subset of $\Gamma_{0}$ defined by

$$
\mathscr{T}_{\Gamma}:=\left\{\alpha \in \Gamma_{0} \mid \operatorname{val}(\alpha)=\mu(\alpha)=1\right\} .
$$

The set of vertices $\mathscr{T}_{\Gamma}$ is just the collection of trucated vertices of $\Gamma$.

Definition 1.3.7. Let $\Gamma=\left(\Gamma_{0}, \Gamma_{1}, \mu, \mathfrak{o}\right)$ be a Brauer configuration. We say that $\Gamma$ is a reduced Brauer configuration, or simply reduced, if satisfies the following property

$$
\forall \alpha \in \mathscr{T}_{\Gamma}, \exists ! V \in \Gamma_{1} ; \alpha \in V \text { and } V \text { is a 2-gon. }
$$


From Definition 1.3.7 we see that the configuration $\Gamma^{*}$ obtained in the reduction process is a reduced Brauer configuration. We call $\Gamma^{*}$ a reduced Brauer configuration associated to $\Gamma$. If $\Gamma$ is a reduced Brauer configuration then is clear that $\Gamma^{*}=\Gamma$. We have the following lemma.

Lemma 1.3.8. [GSa, Lemma 1.9] Let $\Gamma$ be a Brauer configuration and suppose that $\Gamma^{*}$ and $\Gamma^{* *}$ are two reduced configurations associated to $\Gamma$. Then we may choose a relabeling of the vertices of $\Gamma^{* *}$ so that $\Gamma^{*}=\Gamma^{* *}$.

Lemma 1.3.8 allows us to talk about "the" reduced configuration associated to a Brauer configuration. Let $\Gamma^{*}$ be the reduced Brauer Brauer configuration associated to $\Gamma$. After starting a reduction process on the Brauer configuration $\Gamma$, the number of times that we repeat the process until we obtain the reduced Brauer configuration is equal to

$$
\sum_{V \in \Gamma_{1}}\left|V \cap \mathscr{T}_{\Gamma}\right|-\left|\mathscr{T}_{\Gamma^{*}}\right|
$$

So, the reduded Brauer configuration associated to $\Gamma$ has exactly $\left|\Gamma_{0}\right|-\sum_{V \in \Gamma_{1}}\left|V \cap \mathscr{T}_{\Gamma}\right|+\left|\mathscr{T}_{\Gamma^{*}}\right|$ vertices and $\left|\Gamma_{1}\right|$ polygons.

\subsubsection{Brauer configuration algebras}

Let $\Gamma=\left(\Gamma_{0}, \Gamma_{1}, \mu, \mathfrak{o}\right)$ be a Brauer configuration. For each $\alpha \in \Gamma_{0} \backslash \mathscr{T}_{\Gamma}$ consider the set

$$
\mathscr{V}_{(\alpha)}:=\left\{V \in \Gamma_{1} \mid \alpha \in V\right\}
$$

Now consider the list of polygons that belong to $\mathscr{V}_{(\alpha)}$ with $V \operatorname{occurring} \operatorname{occ}(\alpha, V)$ times in this list for each $V \in \mathscr{V}_{(\alpha)}$. As we mentioned in Subsection 1.3.1 the orientation o provides a cyclic ordering of this list. We call such a cyclically ordered list the successor sequence at $\alpha$. We consider the following subsets of $\Gamma_{0} \backslash \mathscr{T}_{\Gamma}$.

$$
\begin{aligned}
& \mathscr{C}_{\Gamma}:=\left\{\alpha \in \Gamma_{0} \mid \operatorname{val}(\alpha)=1 \text { and } \mu(\alpha)>1\right\}, \\
& \mathscr{D}_{\Gamma}:=\left\{\alpha \in \Gamma_{0} \mid \operatorname{val}(\alpha)>1\right\} .
\end{aligned}
$$

We can see that $\Gamma_{0} \backslash \mathscr{T}_{\Gamma}=\mathscr{C}_{\Gamma} \cup \mathscr{D}_{\Gamma}$. Now, for $\alpha \in \mathscr{D}_{\Gamma}$ and $\operatorname{val}(\alpha)=t$ we denote by $\alpha$ : $V_{1}<\cdots<V_{t}$ the successor sequence at $\alpha$. We say that $V_{i+1}$ is the successor of $V_{i}$ at $\alpha$, for $1 \leq i \leq t$, where $V_{t+1}=V_{1}$. Note that for the case $\alpha \in \mathscr{C}_{\Gamma}$ there exists a unique polygon $V$ such that $\alpha \in V$, then the successor sequence at $\alpha$ is just $V$, or for short $\alpha: V$, and by adding $V<V$ we see that $V$ is itself its own successor at $\alpha$. Observe that we also have that $\mathscr{V}_{(\alpha)}=\{V\}$.

Example 1.3.9. As examples of successor sequences we refer to those given in Example 1.3.3. For instance, in $\Gamma$ the successor sequence of vertex 1 is $1: V_{1}<V_{4}<V_{2}$. For the other example, that one given by $\Gamma^{\prime}$, the successor sequence for the vertex $\alpha$ is $\alpha: V<V<V<W$ (or $\alpha: W<V<V<V$ ).

Given a Brauer configuration $\Gamma=\left(\Gamma_{0}, \Gamma_{1}, \mu, \mathfrak{o}\right)$ we define the quiver $\mathcal{Q}_{\Gamma}$ as follows. The vertex set $\left\{v_{1}, \ldots, v_{m}\right\}$ of $\mathcal{Q}_{\Gamma}$ is in bijection with the set of polygons $\left\{V_{1}, \ldots, V_{m}\right\}$ in $\Gamma_{1}$, noting that there is exactly one vertex in $\mathcal{Q}_{\Gamma}$ for every polygon in $\Gamma_{1}$. We call $v_{i}$ (resp. $V_{i}$ ) the vertex (resp. polygon) associated to $V_{i}$ (resp. $v_{i}$ ). In order to define the arrows in $\mathcal{Q}_{\Gamma}$, we use the successor sequences. For each $\alpha \in \Gamma_{0} \backslash \mathscr{T}_{\Gamma}$, and each successor $V^{\prime}$ of $V$ at $\alpha$, there is an arrow from $v$ to $v^{\prime}$ in $\mathcal{Q}_{\Gamma}$, where $v$ and $v^{\prime}$ are the vertices in $\mathcal{Q}_{\Gamma}$ associated to the polygons 
$V$ and $V^{\prime}$ in $\Gamma_{1}$, respectively.

We should note that $V^{\prime}$ can be successor of $V$ more than once at a given vertex of $\Gamma_{0}$, and also that $V^{\prime}$ can be successor of $V$ at more than one vertex of $\Gamma_{0}$. For each such occurrence there is an arrow from $v$ to $v^{\prime}$. In particular, $\mathcal{Q}_{\Gamma}$ may have multiple arrows from $v$ to $v^{\prime}$.

Example 1.3.10. Let's consider the Brauer configurations obtained initially from the tuples in Example 1.3.1 and the multiplicity and order defined in Examples 1.3.2 and 1.3.3, respectively, and which we denoted by $\Gamma=\left(\Gamma_{0}, \Gamma_{1}, \mu, \mathfrak{o}(\Gamma)\right)$ and $\Gamma^{\prime}=\left(\Gamma_{0}^{\prime}, \Gamma_{1}^{\prime}, \mu^{\prime}, \mathfrak{o}\left(\Gamma^{\prime}\right)\right)$. According to the order defined on the list of each nontruncated vertex from $\Gamma$, recall that we took $V_{1}<V_{4}<V_{2}$ as the ordered list of polygons at vertex 1 . Then its successor sequence yields the arrows $a_{1}^{(1)}, a_{2}^{(1)}$ and $a_{3}^{(1)}$ in the quiver (1.7). For example, $V_{4}$ is the successor of $V_{1}$ at vertex 1 yielding the arrow $a_{1}^{(1)}$. The successor sequence $2: V_{1}<V_{2}<V_{3}$ yields the arrows $a_{1}^{(2)}, a_{2}^{(2)}$ and $a_{3}^{(2)}$, and the successor sequence $3: V_{1}<V_{3}<V_{4}$ the arrows $a_{1}^{(3)}, a_{2}^{(3)}, a_{3}^{(3)}$. The quiver associated to $\left(\Gamma_{0}, \Gamma_{1}, \mu, \mathfrak{o}(\Gamma)\right)$ is

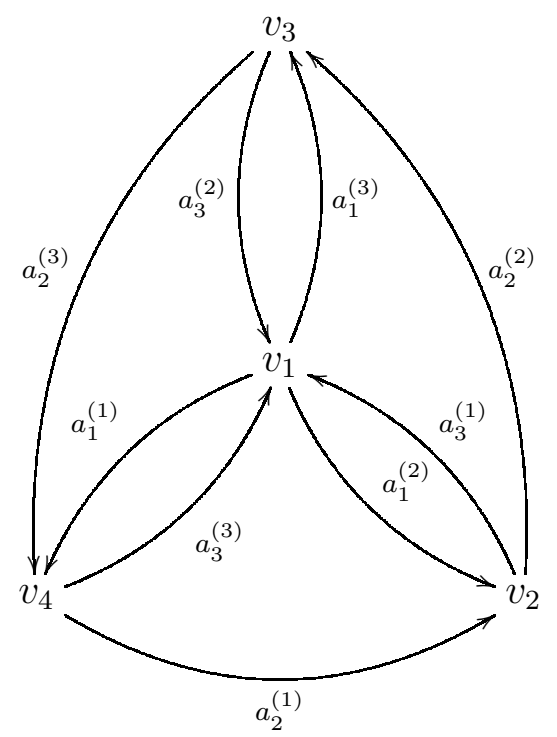

For the orientation $\mathfrak{o}\left(\Gamma^{\prime}\right)$ we have that $\alpha: V<V<V<W$ is the successor sequence at vertex $\alpha$ and yields the arrows $a_{1}^{(\alpha)}, a_{2}^{(\alpha)}, a_{3}^{(\alpha)}$ and $a_{4}^{(\alpha)}$ (observe that $V$ is its own successor at $\alpha$, more than once). The successor sequence at vertex $\beta$ yields the arrows $a_{1}^{(\beta)}, a_{2}^{(\beta)}, a_{3}^{(\beta)}, a_{4}^{(\beta)}$. The quiver associated to $\left(\Gamma_{0}^{\prime}, \Gamma_{1}^{\prime}, \mu^{\prime}, \mathfrak{o}\left(\Gamma^{\prime}\right)\right)$ is

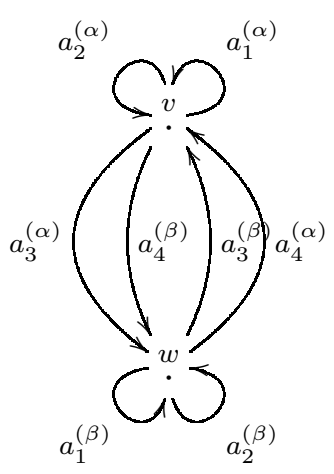

Now we are going to set the relations in the path algebra $K \mathcal{Q}_{\Gamma}$ which will generate the ideal 
used to define the Brauer configuration algebra associated to the Brauer configuration $\Gamma$. These relations are of three types. First we need some extra definitions.

For each $\alpha \in \mathscr{D}_{\Gamma}$ with successor sequence $\alpha: V_{i_{1}}<\cdots<V_{i_{\mathrm{val}(\alpha)}}$ we have the collection of arrows in $\mathcal{Q}_{\Gamma}$

$$
v_{i_{1}} \stackrel{a_{j_{1}}^{(\alpha)}}{\longrightarrow} v_{i_{2}} \stackrel{a_{j_{2}}^{(\alpha)}}{\longrightarrow} \cdots \stackrel{a_{j_{\mathrm{val}(\alpha)}(\alpha)}^{\longrightarrow}}{\longrightarrow} v_{i_{\mathrm{val}(\alpha)}} \stackrel{a_{j_{\mathrm{val}}(\alpha)}^{(\alpha)}}{\longrightarrow} v_{i_{1}}
$$

Let $C_{l}=a_{j_{l}}^{(\alpha)} a_{j_{l+1}}^{(\alpha)} \cdots a_{j_{\mathrm{val}(\alpha)}^{(\alpha)}}^{(\alpha)} \cdots a_{j_{l-1}}^{(\alpha)}$ be the oriented cycle in $\mathcal{Q}_{\Gamma}$, for every $1 \leq l \leq \operatorname{val}(\alpha)$. We call any of these cycles a special $\alpha$-cycle. Now fix a polygon $V \in \mathscr{V}_{(\alpha)}$ and suppose that $\operatorname{occ}(\alpha, V)=t \geq 1$. Then there are $t$ indices $l_{1}, \ldots, l_{t}$ such that $V=V_{i_{l}}$, for every $1 \leq r \leq t$. We call any of the cycles $C_{l_{1}}, \ldots, C_{l_{t}}$ a special $\alpha$-cycle at $v$, and we denote the collection of these cycles in $\mathcal{Q}_{\Gamma}$ by $\mathscr{C}_{(\alpha)}^{v}$. Note that

- $\left|\mathscr{C}_{(\alpha)}^{v}\right|=\operatorname{occ}(\alpha, V)$;

- any element of $\mathscr{C}_{(\alpha)}^{v}$ is a cycle in $\mathcal{Q}_{\Gamma}$ beginning and ending at the vertex $v$;

If $\mathscr{C} \mathscr{C}_{(\alpha)}$ denote the collection of all special $\alpha$-cycles then we can see that

$$
\mathscr{C} \mathscr{C}_{(\alpha)}=\bigcup_{V \in \mathscr{V}_{(\alpha)}} \mathscr{C}_{(\alpha)}^{v}
$$

For the particular case $\alpha \in \mathscr{C}_{\Gamma}$ we define $\mathscr{C} \mathscr{C}_{(\alpha)}$ as the set formed by the unique loop associated to the vertex $\alpha$. In general we have that any two elements in $\mathscr{C} \mathscr{C}_{(\alpha)}$ are cyclic permutations of each other for every $\alpha \in \Gamma_{0} \backslash \mathscr{T}_{\Gamma}$.

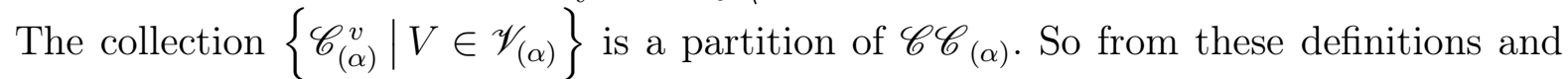
observations we have that for any $\alpha \in \Gamma_{0} \backslash \mathscr{T}_{\Gamma}$

$$
\begin{aligned}
\mid \mathscr{C}_{\mathscr{C}_{(\alpha)} \mid} & =\sum_{V \in \mathscr{V}_{\alpha}}\left|\mathscr{C}_{(\alpha)}^{v}\right| \\
& =\sum_{V \in \mathscr{V}_{(\alpha)}} \operatorname{occ}(\alpha, V) \\
& =\sum_{V \in \Gamma_{1}} \operatorname{occ}(\alpha, V) \\
& =\operatorname{val}(\alpha) .
\end{aligned}
$$

Example 1.3.11. From the quiver in (1.7) induced by the Brauer configuration $\Gamma$ we can see that for every $\alpha \in \Gamma_{0}$ there is only one $\alpha$-cycle at $v$ for each $V \in \mathscr{V}_{(\alpha)}$. So, for example, for the vertex 2 in $\Gamma_{0}$ we have $\mathscr{V}_{(2)}=\left\{V_{1}, V_{2}, V_{3}\right\}$ then

$$
\begin{aligned}
& \mathscr{C}_{(2)}^{v_{1}}=\left\{a_{1}^{(2)} a_{2}^{(2)} a_{3}^{(2)}\right\} \\
& \mathscr{C}_{(2)}^{v_{2}}=\left\{a_{2}^{(2)} a_{3}^{(2)} a_{1}^{(2)}\right\} \\
& \mathscr{C}_{(2)}^{v_{3}}=\left\{a_{3}^{(2)} a_{1}^{(2)} a_{2}^{(2)}\right\}
\end{aligned}
$$

hence the collection of all the 2-cycles is

$$
\begin{aligned}
\mathscr{C}_{\mathscr{C}_{(2)}} & =\mathscr{C}_{(2)}^{v_{1}} \cup \mathscr{C}_{(2)}^{v_{2}} \cup \mathscr{C}_{(2)}^{v_{3}} \\
& =\left\{a_{1}^{(2)} a_{2}^{(2)} a_{3}^{(2)}, a_{2}^{(2)} a_{3}^{(2)} a_{1}^{(2)}, a_{3}^{(2)} a_{1}^{(2)} a_{2}^{(2)}\right\}
\end{aligned}
$$


Example 1.3.12. For the quiver in (1.8) induced by the Brauer configuration $\Gamma^{\prime}$ we obtain a different situation. At this case the vertices are repeating more than once in some polygon of the configuration $\Gamma^{\prime}$, i.e, we have $\operatorname{occ}(\alpha, V)=\operatorname{occ}(\beta, W)=3$. For example, for the vertex $\beta \in \Gamma_{0}^{\prime}$ we have $\mathscr{V}_{(\beta)}=\{V, W\}$ and

$$
\begin{aligned}
\mathscr{C}_{(\beta)}^{w} & =\left\{a_{1}^{(\beta)} a_{2}^{(\beta)} a_{3}^{(\beta)} a_{4}^{(\beta)}, a_{2}^{(\beta)} a_{3}^{(\beta)} a_{4}^{(\beta)} a_{1}^{(\beta)}, a_{3}^{(\beta)} a_{4}^{(\beta)} a_{1}^{(\beta)} a_{2}^{(\beta)}\right\} \\
\mathscr{C}_{(\beta)}^{v} & =\left\{a_{4}^{(\beta)} a_{1}^{(\beta)} a_{2}^{(\beta)} a_{3}^{(\beta)}\right\}
\end{aligned}
$$

which implies

$$
\begin{aligned}
\mathscr{C}_{(\beta)} & =\mathscr{C}_{(\beta)}^{w} \cup \mathscr{C}_{(\beta)}^{v} \\
& =\left\{a_{1}^{(\beta)} a_{2}^{(\beta)} a_{3}^{(\beta)} a_{4}^{(\beta)}, a_{2}^{(\beta)} a_{3}^{(\beta)} a_{4}^{(\beta)} a_{1}^{(\beta)}, a_{3}^{(\beta)} a_{4}^{(\beta)} a_{1}^{(\beta)} a_{2}^{(\beta)}, a_{4}^{(\beta)} a_{1}^{(\beta)} a_{2}^{(\beta)} a_{3}^{(\beta)}\right\}
\end{aligned}
$$

Before giving the definition of a Brauer configuration algebra we need one last thing. Let $\mathscr{C} \mathscr{C}$ be the set of special cycles in $\mathcal{Q}_{\Gamma}$ defined by

$$
\mathscr{C} \mathscr{C}=\bigcup_{\alpha \in \Gamma_{0} \backslash \mathscr{T}_{\Gamma}} \mathscr{C} \mathscr{C}_{(\alpha)}
$$

and let $f: \mathscr{C} \mathscr{C} \rightarrow\left(\mathcal{Q}_{\Gamma}\right)_{1}$ be the map which sends a special cycle to its first arrow.

We now define a set of elements $\rho_{\Gamma}$ in $K \mathcal{Q}_{\Gamma}$ which will generate the ideal of relations $I_{\Gamma}$ of the Brauer configuration algebra associated to the Brauer configuration $\Gamma$. Define three types of relations in $K \mathcal{Q}_{\Gamma}$ by setting:

Relations of type one. It is the subset of $K \mathcal{Q}_{\Gamma}$

$$
\bigcup_{V \in \Gamma_{1}}\left(\bigcup_{\alpha, \beta \in V \backslash \mathscr{T}_{\Gamma}}\left\{C^{\mu(\alpha)}-D^{\mu(\beta)} \mid C \in \mathscr{C}_{(\alpha)}^{v}, D \in \mathscr{C}_{(\beta)}^{v}\right\}\right)
$$

Relations of type two. It is the subset of $K \mathcal{Q}_{\Gamma}$

$$
\bigcup_{\alpha \in \Gamma_{0} \backslash \mathscr{T}_{\Gamma}}\left\{C^{\mu(\alpha)} f(C) \mid C \in \mathscr{C} \mathscr{C}_{(\alpha)}\right\}
$$

Relations of type three. It is the set of all quadratic monomial relations of the form $a b$ in $K \mathcal{Q}_{\Gamma}$ where $a b$ is not a subpath of any special cycle.

Definition 1.3.13. Let $K$ be a field and $\Gamma$ a Brauer configuration. The Brauer configuration algebra $\Lambda_{\Gamma}$ associated to $\Gamma$ is defined to be $K \mathcal{Q}_{\Gamma} / I_{\Gamma}$, where $\mathcal{Q}_{\Gamma}$ is the quiver associated to $\Gamma$ and $I_{\Gamma}$ is the ideal in $K \mathcal{Q}_{\Gamma}$ generated by the set of relations $\rho_{\Gamma}$ of type one, two and three.

We should mention that the collection of relations $\rho_{\Gamma}$ generating the ideal $I_{\Gamma}$ is not necessarily minimal and usually contains redundant relations.

Example 1.3.14. Let $\Gamma^{\prime \prime \prime}=\left(\Gamma_{0}^{\prime \prime \prime}, \Gamma_{1}^{\prime \prime \prime}, \mu^{\prime \prime \prime}, \mathfrak{o}^{\prime \prime \prime}\right)$ be the Brauer configuration given by $\Gamma_{0}^{\prime \prime \prime}=$ $\{1\}, \Gamma_{1}^{\prime \prime \prime}=\{V\}$ with $V=\{1,1\}, \mu^{\prime \prime \prime} \equiv 1$ and order $\mathfrak{o}^{\prime \prime \prime}$ defined by the successor sequence $1: V<V$. Let $\mathcal{Q}^{\prime \prime \prime}$ be the induced quiver by $\Gamma^{\prime \prime \prime}$ with arrows $a_{1}^{(1)}$ and $a_{2}^{(1)}$. Then $\mathcal{Q}^{\prime \prime \prime}$ is

$$
a_{2}^{(1)} \bigcup^{\supset} a_{1}^{(1)}
$$


We observe that there are only two special cycles: $a_{1}^{(1)} a_{2}^{(1)}$ and $a_{2}^{(1)} a_{1}^{(1)}$. The defining relations for the Brauer configuration algebra induced by $\Gamma^{\prime \prime \prime}$ are

- Relations of type one:

$$
a_{1}^{(1)} a_{2}^{(1)}-a_{2}^{(1)} a_{1}^{(1)}
$$

- Relations of type two:

$$
a_{1}^{(1)} a_{2}^{(1)} a_{1}^{(1)} \text { and } a_{2}^{(1)} a_{1}^{(1)} a_{2}^{(1)}
$$

- Relations of type three:

$$
\left(a_{1}^{(1)}\right)^{2} \text { and }\left(a_{2}^{(1)}\right)^{2} \text {. }
$$

If $\Lambda_{\Gamma^{\prime \prime \prime}}$ is the induced Brauer configuration algebra, then by the relation of type one we can see that this algebra is commutative. It is not difficult to prove that the Brauer configuration algebra of this example is isomorphic to the algbera $K\left[x_{1}, x_{2}\right] /\left\langle x_{1}^{2}, x_{2}^{2}\right\rangle$.

Example 1.3.15. Consider the quiver in (1.7) induced by the Brauer configuration $\Gamma$ in Example 1.3.10. We can compute a minimal set of relations for the ideal $I_{\Gamma}$ of the Brauer configuration algebra induced by $\Gamma$. This set is given by the following relations

- Relations of type one:

$$
\begin{aligned}
& \left(a_{2}^{(2)} a_{3}^{(2)} a_{1}^{(2)}\right)^{N}-\left(a_{3}^{(1)} a_{1}^{(1)} a_{2}^{(1)}\right)^{N} \\
& \left(a_{2}^{(3)} a_{3}^{(3)} a_{1}^{(3)}\right)^{N}-\left(a_{3}^{(2)} a_{1}^{(2)} a_{2}^{(2)}\right)^{N} \\
& \left(a_{2}^{(1)} a_{3}^{(1)} a_{1}^{(1)}\right)^{N}-\left(a_{3}^{(3)} a_{1}^{(3)} a_{2}^{(3)}\right)^{N}, \\
& \left(a_{1}^{(3)} a_{2}^{(3)} a_{3}^{(3)}\right)^{N}-\left(a_{1}^{(1)} a_{2}^{(1)} a_{3}^{(1)}\right)^{N} \\
& \left(a_{1}^{(2)} a_{2}^{(2)} a_{3}^{(2)}\right)^{N}-\left(a_{1}^{(3)} a_{2}^{(3)} a_{3}^{(3)}\right)^{N} .
\end{aligned}
$$

- Relations of type two:

$$
\begin{aligned}
& \left(a_{1}^{(1)} a_{2}^{(1)} a_{3}^{(1)}\right)^{N} a_{1}^{(1)},\left(a_{2}^{(1)} a_{3}^{(1)} a_{1}^{(1)}\right)^{N} a_{2}^{(1)},\left(a_{3}^{(1)} a_{1}^{(1)} a_{2}^{(1)}\right)^{N} a_{3}^{(1)}, \\
& \left(a_{1}^{(2)} a_{2}^{(2)} a_{3}^{(2)}\right)^{N} a_{1}^{(2)},\left(a_{2}^{(2)} a_{3}^{(2)} a_{1}^{(2)}\right)^{N} a_{2}^{(2)},\left(a_{3}^{(2)} a_{1}^{(2)} a_{2}^{(2)}\right)^{N} a_{3}^{(2)}, \\
& \left(a_{1}^{(3)} a_{2}^{(3)} a_{3}^{(3)}\right)^{N} a_{1}^{(3)},\left(a_{2}^{(3)} a_{3}^{(3)} a_{1}^{(3)}\right)^{N} a_{2}^{(3)},\left(a_{3}^{(3)} a_{1}^{(3)} a_{2}^{(3)}\right)^{N} a_{3}^{(3)},
\end{aligned}
$$

- Relations of type three:

$$
\begin{aligned}
& a_{2}^{(1)} a_{2}^{(2)}, a_{1}^{(2)} a_{3}^{(1)}, a_{2}^{(2)} a_{2}^{(3)}, a_{1}^{(3)} a_{3}^{(2)}, a_{1}^{(3)} a_{2}^{(1)}, a_{1}^{(1)} a_{3}^{(3)}, \\
& a_{3}^{(2)} a_{1}^{(1)}, a_{3}^{(2)} a_{1}^{(3)}, a_{3}^{(3)} a_{1}^{(1)}, a_{3}^{(3)} a_{1}^{(2)}, a_{3}^{(1)} a_{1}^{(3)}, a_{3}^{(1)} a_{1}^{(2)} .
\end{aligned}
$$

It is easy to check that the set of relations of type one can generate any other relation of type one in the induced path algbera $K \mathcal{Q}_{\Gamma}$.

The following proposition shows that the reduction procedure for removing truncated vertices from a $d$-gon, with $d>2$, does not change the Brauer configuration algebra.

Proposition 1.3.16. [GSa, Proposition 2.7] Let $\Gamma$ be a Brauer configuration with associated Brauer configuration algebra $\Lambda_{\Gamma}$. Suppose $\alpha \in \Gamma_{0}$ is a truncated vertex in a polygon $V \in \Gamma_{1}$ and $V$ is a $d$-gon, with $d \geq 3$. Let $\Gamma^{\prime}$ the Brauer configuration obtained by applying the reduction procedure of Subsection 1.3.1 for removing the vertex $\alpha$. If $\Lambda_{\Gamma^{\prime}}$ is the induced Brauer configuration algebra of $\Gamma^{\prime}$, then the algebras $\Lambda_{\Gamma}$ and $\Lambda_{\Gamma^{\prime}}$ are isomorphic. 
Proof. Since $\alpha$ is truncated, there is a unique polygon $V \in \Gamma_{1}$ such that $\alpha \in V$. Because $\alpha$ is truncated there are no special cycles associated to $\alpha$. So in the quiver of $\Lambda_{\Gamma}$ there are no arrows created by $\alpha$. In fact, the quivers of $\Lambda_{\Gamma}$ and $\Lambda_{\Gamma^{\prime}}$ are exactly the same and the ideals of relations are the same in the respective path algebras, so the associated algebras also are the same.

Because of Proposition 1.3.16 we may assume from now on that all the Brauer configurations are reduced. For $\Gamma$ a reduced Brauer configuration, let $2-\mathscr{P}_{\Gamma}$ be the subset of $\Gamma_{1}$ given by

$$
2-\mathscr{P}_{\Gamma}:=\left\{V \in \Gamma_{1}|| V \mid=2 \text { and } V \cap \mathscr{T}_{\Gamma} \neq \emptyset\right\} .
$$

The set $2-\mathscr{P}_{\Gamma}$ is simply the collection of all 2 -gons of $\Gamma_{1}$ that have one truncated vertex.

Definition 1.3.17. Let $\Gamma=\left(\Gamma_{0}, \Gamma_{1}, \mu, \mathfrak{o}\right)$ be a Brauer configuration such that each polygon in $\Gamma_{1}$ is a 2-gon. Then we say that $\Gamma$ is a Brauer graph. The induced algebra induced by the configuration $\Gamma$ is called a Brauer graph algebra.

Every Brauer graph has a particular graphical way to represent almost all its combinatorial data. For an arbitrary Brauer configuration we may also represent its combinatorial data in a graphical way (see [GSa, Section 1.2]). Let's see the following examples.

Example 1.3.18. Consider the unoriented graph

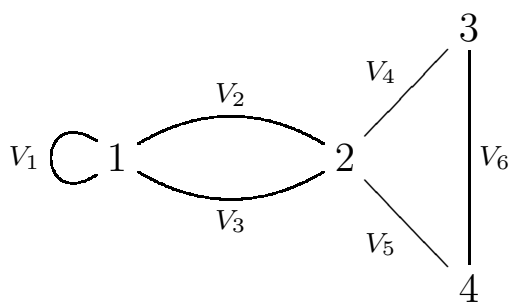

Let $\Gamma$ be the Brauer configuration defined by

- $\Gamma_{0}=\{1,2,3,4\}$

- $\Gamma_{1}=\left\{V_{1}, V_{2}, V_{3}, V_{4}, V_{5}, V_{6}\right\}$ where

$$
\begin{aligned}
& V_{1}=\{1,1\}, \quad V_{4}=\{2,3\}, \\
& V_{2}=\{1,2\}, \quad V_{5}=\{2,4\}, \\
& V_{3}=\{1,2\}, \quad V_{6}=\{3,4\} .
\end{aligned}
$$

Observe that in the way that we are defining the polygons of $\Gamma$, we are simply representing each polygon by an edge of the unoriented graph in (1.14). Also observe that the loop in (1.14) is represented by a 2-gon with the vertex 1 repeated twice, and parallel edges are just 2-gons with the same set of vertices.

- With this representation polygon-edge, orientation $\mathfrak{o}$ over the list of polygons of a vertex can be defined as a cyclic ordering of the edges incident at the vertex. So, for the vertex 2 this orientation gives the successor sequence at 2

$$
2: V_{4}<V_{5}<V_{3}<V_{2}
$$

And for vertices 3 and 4

$$
3: V_{6}<V_{4}
$$




$$
4: V_{6}<V_{5} \text {. }
$$

In vertex 1 , where we have a loop, we may choose the successor sequence

$$
1: V_{1}<V_{1}<V_{2}<V_{3} \text {. }
$$

We could also choose a different successor sequence at vertex 1 , for example $1: V_{1}<$ $V_{2}<V_{1}<V_{3}$. The 2-gon $V_{1}$ must appear twice in any successor sequence at 1 . But for this example we will remain with the successor sequence in (1.15).

- Let $\mu: \Gamma_{0} \rightarrow \mathbb{Z}^{>0}$ be any map.

With these data we have the Brauer configuration $\Gamma=\left(\Gamma_{0}, \Gamma_{1}, \mu, \mathfrak{o}\right)$ which is by definition a Brauer graph. We observe that $\Gamma$ has no truncated vertex.

Example 1.3.19. Let $\Gamma^{\prime \prime}=\left(\Gamma_{0}^{\prime \prime}, \Gamma_{1}^{\prime \prime}, \mu^{\prime \prime}, \mathfrak{o}^{\prime \prime}\right)$ be the Brauer graph given by

- $\Gamma_{0}^{\prime \prime}=\{1,2,3,4\}$.

- $\Gamma_{1}^{\prime \prime}=\left\{V_{1}, V_{2}, V_{3}, V_{4}\right\}$ with

$$
\begin{aligned}
& V_{1}=\{1,3\}, \quad V_{3}=\{1,2\}, \\
& V_{2}=\{2,3\}, \quad V_{4}=\{1,4\} .
\end{aligned}
$$

- $\mu^{\prime \prime} \equiv 1$.

- The orientation $\mathfrak{o}^{\prime \prime}$ gives the following successor sequence at the nontruncated vertices

$$
\begin{array}{ll}
1: & V_{1}<V_{3}<V_{4}, \\
2: & V_{3}<V_{2}, \\
3: & V_{1}<V_{2} .
\end{array}
$$

Observe that vertex 4 is a truncated vertex. All these data can be represented by the following unoriented graph

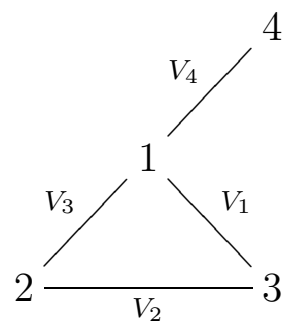

where the cyclic ordering of the edges incident in a vertex is taken counterclockwise. Then the induced quiver is

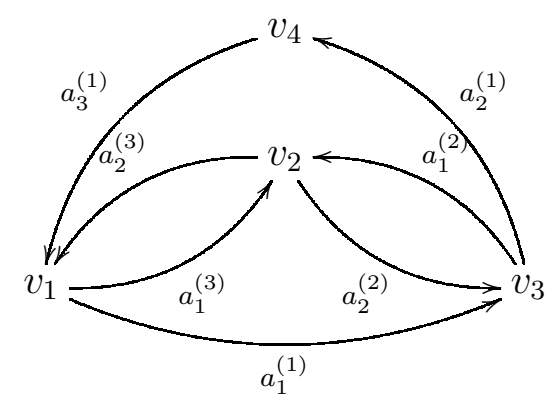


Proposition 1.3.20. Let $\Gamma=\left(\Gamma_{0}, \Gamma_{1}, \mu, \mathfrak{o}\right)$ be a reduced Brauer configuration and let $\Lambda_{\Gamma}$ be the associated Brauer configuration algebra. If $\mathscr{T}_{\Gamma}=\emptyset$ then, the ideal $I_{\Gamma}$ can be generated by relations of type one and type three only.

Proof. If $\mathscr{T}_{\Gamma}=\emptyset$ then the Brauer configuration $\Gamma$ doesn't have any truncated vertex. Because relations of type three are quadratic monomial necessarily they must be contained in any minimal generating set for $I_{\Gamma}$. Now, for $\alpha$ any vertex of $\Gamma$ consider the set $\mathscr{V}_{(\alpha)}$. If $V \in \mathscr{V}_{(\alpha)}$ we have that $|V| \geq 2$ and no vertex in $V$ is truncated. For $\beta \in V$ ( $\beta$ may be equal to $\alpha$ in case that $\operatorname{occ}(\alpha, V)>1)$ and $v$ the vertex in $\mathcal{Q}_{\Gamma}$ associated to $V$, let $C \in \mathscr{C}_{(\alpha)}^{v}$ and $D \in \mathscr{C}_{(\beta)}^{v}$ be different special cycles. So, we have that $C^{\mu(\alpha)}-D^{\mu(\beta)}$ is a relation in $\rho_{\Gamma}$, hence $C^{\mu(\alpha)} f(C)-D^{\mu(\beta)} f(C)=\left(C^{\mu(\alpha)}-D^{\mu(\beta)}\right) f(C) \in I_{\Gamma}$ but the monomial $D^{\mu(\beta)} f(C)$ contains a relation of type three as a subpath, which implies that $C^{\mu(\alpha)} f(C) \in I_{\Gamma}$, so we see that any relation of type two can be generated using only relations of type one and relations of type three and hence any minimal generating set for $I_{\Gamma}$ must contain these type of relations.

The converse of Proposition 1.3.20 it is false.

Example 1.3.21. Let $\Gamma^{\prime \prime}$ be the Brauer graph of Example 1.3.19. We obseved there that this Brauer graph has the vertex 4 as a truncated vertex. From the quiver in (1.17) we see that we have only three relations of type one

$$
\begin{gathered}
a_{1}^{(1)} a_{2}^{(1)} a_{3}^{(1)}-a_{1}^{(3)} a_{2}^{(3)}, \\
a_{2}^{(1)} a_{3}^{(1)} a_{1}^{(1)}-a_{1}^{(2)} a_{2}^{(2)}, \\
a_{2}^{(3)} a_{1}^{(3)}-a_{2}^{(2)} a_{1}^{(2)},
\end{gathered}
$$

and six relations of type three

$$
\begin{aligned}
& a_{3}^{(1)} a_{1}^{(3)}, a_{2}^{(3)} a_{1}^{(1)}, a_{1}^{(1)} a_{1}^{(2)}, \\
& a_{2}^{(2)} a_{2}^{(1)}, a_{1}^{(2)} a_{2}^{(3)}, a_{1}^{(3)} a_{2}^{(2)} .
\end{aligned}
$$

There are 7 relations of type two ${ }^{1}$. We can calculate all the relations of type two using only the relations above, i.e, using relations of type one and relations of type three only. Indeed

$$
\begin{aligned}
a_{1}^{(1)} a_{2}^{(1)} a_{3}^{(1)} a_{1}^{(1)} & \left.={ }_{1}^{(1)} a_{2}^{(1)} a_{3}^{(1)}-a_{1}^{(3)} a_{2}^{(3)}\right) a_{1}^{(1)}+a_{1}^{(3)} a_{2}^{(3)} a_{1}^{(1)}, \\
a_{2}^{(1)} a_{3}^{(1)} a_{1}^{(1)} a_{2}^{(1)} & =\left(a_{2}^{(1)} a_{3}^{(1)} a_{1}^{(1)}-a_{1}^{(2)} a_{2}^{(2)}\right) a_{2}^{(1)}+a_{1}^{(2)} a_{2}^{(2)} a_{2}^{(1)}, \\
a_{3}^{(1)} a_{1}^{(1)} a_{2}^{(1)} a_{3}^{(1)} & =a_{3}^{(1)}\left(a_{1}^{(1)} a_{2}^{(1)} a_{3}^{(1)}-a_{1}^{(3)} a_{2}^{(3)}\right)+a_{3}^{(1)} a_{1}^{(3)} a_{2}^{(3)}, \\
a_{1}^{(3)} a_{2}^{(3)} a_{1}^{(3)} & =-\left(a_{1}^{(1)} a_{2}^{(1)} a_{3}^{(1)}-a_{1}^{(3)} a_{2}^{(3)}\right) a_{1}^{(3)}+a_{1}^{(1)} a_{2}^{(1)} a_{3}^{(1)} a_{1}^{(3)}, \\
a_{1}^{(2)} a_{2}^{(2)} a_{1}^{(2)} & =-\left(a_{2}^{(1)} a_{3}^{(1)} a_{1}^{(1)}-a_{1}^{(2)} a_{2}^{(2)}\right) a_{1}^{(2)}+a_{2}^{(1)} a_{3}^{(1)} a_{1}^{(1)} a_{1}^{(2)}, \\
a_{2}^{(3)} a_{1}^{(3)} a_{2}^{(3)} & =\left(a_{2}^{(3)} a_{1}^{(3)}-a_{2}^{(2)} a_{1}^{(2)}\right) a_{2}^{(3)}+a_{2}^{(2)} a_{1}^{(2)} a_{2}^{(3)}, \\
a_{2}^{(2)} a_{1}^{(2)} a_{2}^{(2)} & =-\left(a_{2}^{(3)} a_{1}^{(3)}-a_{2}^{(2)} a_{1}^{(2)}\right) a_{2}^{(2)}+a_{2}^{(3)} a_{1}^{(3)} a_{2}^{(2)} .
\end{aligned}
$$

Then we have that the ideal $I_{\Gamma^{\prime \prime}}$ can be generated using relations of type one and relations of type three only but $\mathscr{T}_{\Gamma^{\prime \prime}} \neq \emptyset$.

The following proposition will tell us when any minimal generating set of the ideal $I_{\Gamma}$ of a Brauer configuration $\Gamma$ must contain a relation of type two.

\footnotetext{
${ }^{1}$ The total number of relations of type two for a Brauer configuration algebra always coincides with the number of arrows in the induced quiver.
} 
Proposition 1.3.22. Let $\Gamma=\left(\Gamma_{0}, \Gamma_{1}, \mu, \mathfrak{o}\right)$ be a reduced Brauer configuration and let $\Lambda_{\Gamma}$ be the induced Brauer configuration algebra. For $\alpha \in \Gamma_{0} \backslash \mathscr{T}_{\Gamma}$ let $V \in \mathscr{V}_{(\alpha)}$ such that $V \in 2-\mathscr{P}$ and let $W$ be the successor of $V$ at $\alpha$, and let $\rho$ be a minimal generating set for the ideal $I_{\Gamma}$. If $C \in \mathscr{C}_{(\alpha)}^{v}$ then $C^{\mu(\alpha)} f(C) \in \rho$ if and only if $W \in 2-\mathscr{P}$.

Proof. See [GSST, Lemma 2.1]. Apply the same reasoning.

\subsubsection{Special cycles}

In this subsection we are going to consider special cycles of Brauer configuration algebras and some multiplicative properties that depend on these special cycles. If no confusion arises we will denote by $\Lambda=K \mathcal{Q} / I$ the associated Brauer configuration algebra of the reduced Brauer configuration $\Gamma$.

As we said in Section 1.2 we denote by $\bar{x}$ the class of $x$ in $\Lambda=K \mathcal{Q} / I$. If $\pi: K \mathcal{Q} \rightarrow \Lambda$ is the canonical surjection then we have that $\pi(x)=\bar{x}$, for any $x \in K \mathcal{Q}$.

Recall that for $\alpha \in \Gamma_{0} \backslash \mathscr{T}_{\Gamma}$ and $V \in \mathscr{V}_{(\alpha)}$ we denote the set of all special $\alpha$-cycles at $v$ by $\mathscr{C}_{(\alpha)}^{v}$, where $v$ is the vertex in $\mathcal{Q}$ associated to $V$. Recalling expression (1.10) we have that all the special cycles associated to the vertex $\alpha$ are given by the union

$$
\mathscr{C} \mathscr{C}_{(\alpha)}=\bigcup_{V \in \mathscr{V}_{(\alpha)}} \mathscr{C}_{(\alpha)}^{v}
$$

the collection $\left\{\mathscr{C}_{(\alpha)}^{v} \mid V \in \mathscr{V}_{(\alpha)}\right\}$ is a partition of $\mathscr{C} \mathscr{C}_{(\alpha)}$, and the total set of special cycles in $\mathcal{Q}$ can be partitionated by the collection $\left\{\mathscr{C}_{\mathscr{C}}(\alpha) \mid \alpha \in \Gamma_{0} \backslash \mathscr{T}_{\Gamma}\right\}$. For a $\alpha \in \Gamma_{0} \backslash \mathscr{T}_{\Gamma}$ and $V \in \mathscr{V}_{(\alpha)}$, the number of elements in $\mathscr{C}_{(\alpha)}^{v}$ denotes the number of times that $\alpha$ occurs in $V$, i.e, $\left|\mathscr{C}_{(\alpha)}^{v}\right|=\operatorname{occ}(\alpha, V)$ then for the expression in (1.11) we have that

$$
\left|\mathscr{C} \mathscr{C}_{(\alpha)}\right|=\operatorname{val}(\alpha)
$$

which is the exactly number of arrows of any special $\alpha$-cycle in $\mathcal{Q}$. Now using the partition of the set of special cycles is not difficult to prove that the map $f: \mathscr{C} \mathscr{C} \rightarrow \mathcal{Q}_{1}$ defined in Subsection 1.3.1 is a bijection, from this it follows that

$$
\begin{aligned}
\left|\mathcal{Q}_{1}\right| & =\sum_{\alpha \in \Gamma_{0} \backslash \mathscr{T}_{\Gamma}}\left|\mathscr{C} \mathscr{C}_{(\alpha)}\right| \\
& =\sum_{\alpha \in \Gamma_{0} \backslash \mathscr{T}_{\Gamma}} \operatorname{val}(\alpha) .
\end{aligned}
$$

We have the following proposition.

Proposition 1.3.23. Let $\Gamma$ be a reduced Brauer configuration and let $\mathcal{Q}$ be its induced quiver.

(1) For $a \in \mathcal{Q}_{1}$ there exists a unique $\alpha \in \Gamma_{0} \backslash \mathscr{T}_{\Gamma}$ such that $a$ appears exactly once in each special cycle in $\mathscr{C} \mathscr{C}_{(\alpha)}$.

$$
\left|\mathcal{Q}_{1}\right|=\sum_{\alpha \in \Gamma_{0} \backslash \mathscr{T}_{\Gamma}} \operatorname{val}(\alpha) .
$$


Given $\alpha \in \mathscr{D}_{\Gamma}$ there is a graphical and simple way to represent all the elements in the set $\mathscr{C} \mathscr{C}_{(\alpha)}$. If $\alpha: V_{i_{1}}<\cdots<V_{i_{\mathrm{val}(\alpha)}}$ is the successor sequence at $\alpha$ then in the induced quiver $\mathcal{Q}$ we have a sequence of arrows

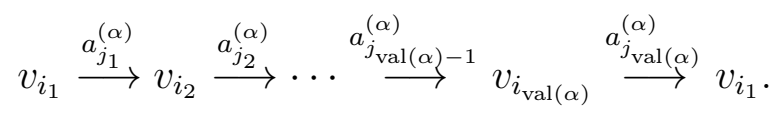

where $v_{i_{l}}$ is the vertex in $\mathcal{Q}$ associated to $V_{i_{l}}$ for every $1 \leq l \leq \operatorname{val}(\alpha)$. Let's put the arrows and vertices in a cyclic draw like this.

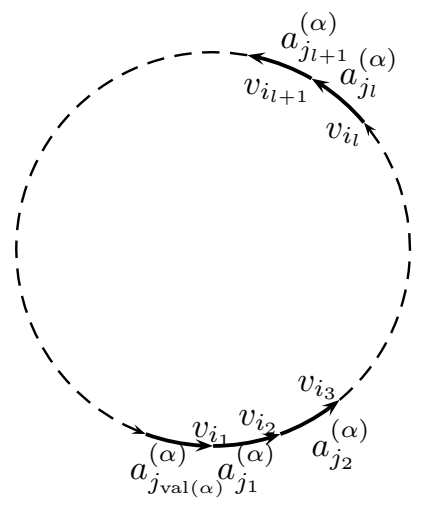

Figure 1.1

At this point it is not yet important to consider if any of the polygons in the successor sequence of $\alpha$ appears more than once. Setting $a_{0}^{(\alpha)}=a_{\mathrm{val}(\alpha)}^{(\alpha)}$, for $1 \leq l \leq \operatorname{val}(\alpha)$ we denote by $C_{l}$ the composition of the arrows from $a_{j_{l}}^{(\alpha)}$ until $a_{j_{l-1}}^{(\alpha)}$, i.e,

$$
C_{l}=a_{j_{l}}^{(\alpha)} a_{j_{l+1}}^{(\alpha)} \cdots a_{j_{l-1}}^{(\alpha)}
$$

Now, in Figure 1.1 replace the vertex $v_{i_{l}}$ by the special $\alpha$-cycle $C_{l}$, for each $1 \leq l \leq \operatorname{val}(\alpha)$. We obtain the following figure.

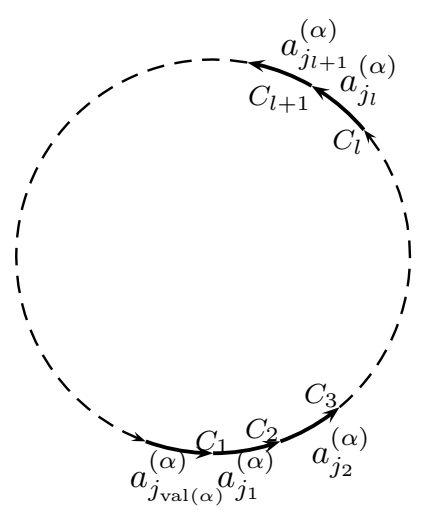

Figure 1.2

The special cycle $C_{l}$ in Figure 1.2 represents a complete round in the circle, starting and ending at the vertex $v_{i_{l}}$, for each $1 \leq l \leq \operatorname{val}(\alpha)$. So we can represent all the elements of $\mathscr{C} \mathscr{C}_{(\alpha)}$ by looking at the Figure 1.2. 
From the definition of the type one relations we see that for a $V \in \Gamma_{1}$ and any $\alpha, \beta \in V \backslash \mathscr{T}_{\Gamma}$ we have

$$
\bar{C}^{\mu(\alpha)}=\bar{D}^{\mu(\beta)}, \forall C \in \mathscr{C}_{(\alpha)}^{v}, \forall D \in \mathscr{C}_{(\beta)}^{v} .
$$

So, let $\alpha$ be any nontruncated vertex in $V$, and let $C \in \mathscr{C}_{(\alpha)}^{v}$. We denote by $C^{(V)}$ any representative of the equivalence class

$$
\left\{C^{\mu(\alpha)}+x \mid x \in I_{\Gamma}\right\}
$$

Then, if $\beta \in V$ is another nontruncated vertex we have

$$
C^{(V)}=\bar{D}^{\mu(\beta)}, \forall D \in \mathscr{C}_{(\beta)}^{v} .
$$

One of the particular features of the multiplicative structure of a Brauer configuration algebra results from the relations of type three. The following proposition is a consequence of that.

Proposition 1.3.24. Let $\Gamma=\left(\Gamma_{0}, \Gamma_{1}, \mu, \mathfrak{o}\right)$ be a reduced Brauer configuration and $\Lambda=$ $K \mathcal{Q} / I$ its associated Brauer configuration algebra. If $\alpha, \beta \in \Gamma_{0} \backslash \mathscr{T}_{\Gamma}$ is such that $\alpha \neq \beta$ then

(1) $\bar{C} \bar{D}=\bar{D} \bar{C}=0$ in $\Lambda, \forall C \in \mathscr{C}_{(\alpha)}, \forall D \in \mathscr{C} \mathscr{C}_{(\beta)}$.

(2) Let $1 \leq s \leq \operatorname{val}(\alpha)$ and $1 \leq t \leq \operatorname{val}(\beta)$. If $C \in \mathscr{C} \mathscr{C}_{(\alpha)}, D \in \mathscr{C} \mathscr{C}_{(\beta)}$, and $p_{1}$ and $p_{2}$ are strictly subpaths of $C^{s}$ and $D^{t}$ respectively, then

$$
\overline{p_{1}} \overline{p_{2}}=\overline{p_{2}} \overline{p_{1}}=0
$$

in $\Lambda$.

The next proposition, and its corollary, also shows the very tight multiplicative structure inside $\Lambda$.

Proposition 1.3.25. Let $\Gamma=\left(\Gamma_{0}, \Gamma_{1}, \mu, \mathfrak{o}\right)$ be a reduced Brauer configuration and $\Lambda=$ $K \mathcal{Q} / I$ its associated Brauer configuration algebra and let $\alpha \in \mathscr{D}_{\Gamma}$, and $C, D \in \mathscr{C}_{(}(\alpha)$ two different special cycles. Using the graphical representation given in Figure 1.2 suppose that $C=C_{k}$ and $D=C_{l}$ with $1 \leq k<l \leq \operatorname{val}(\alpha)$. Let $p$ the path in $\mathcal{Q}$ obtained by compossing all the arrows between $C$ and $D$. Now, for $0 \leq s<\operatorname{val}(\alpha)$ set $x=C^{s} p$ and $y=p D^{s}$. Then

(1) $x=y$ in $\mathcal{Q}$.

(2) $\bar{x} \neq 0$ and $\bar{y} \neq 0$.

(3) If $a$ is an arrow in $\mathcal{Q}$, then

- $\bar{x} \bar{a} \neq 0$ if and only if $a$ is the first arrow of $D$.

- $\bar{a} \bar{y} \neq 0$ if and only if $a$ is the last arrow of $C$.

Proof. Part (1) follows from the graphical representation in Figure 1.2. Since $x$ or $y$ have no subpaths that are type two relations or type three relations we see that (2) holds. Statement (3) follows from the equality $x=y$ in $\mathcal{Q}$ and by Proposition 1.3.23(1) and Proposition 1.3.24. 


\subsubsection{Some properties of Brauer configuration algebras}

In this subsection we are going to prove some basic properties of Brauer configuration algebras. From now on we assume that $\Lambda=K \mathcal{Q} / I$ where $\Lambda$ is a Brauer configuration algebra associated to a Brauer configuration and $\mathcal{Q}$ is the quiver of $\Lambda$. We also assume that all the Brauer configurations are reduced. First we show two basic properties about $\Lambda$.

- $I$ is an admissible ideal.

- $\Lambda$ is a symmetric algebra.

Then we show that a Brauer configuration algebra is indecomposable if and only if its Brauer configuration is connected. All these and more can be found in [GSa].

For any path $p$ in the quiver $\mathcal{Q}$, we let $l(p)$ denote the length of $p$. When we say that a path $p$ in $\mathcal{Q}$ is a prefix of other path $q$ in $\mathcal{Q}$, we mean that the first arrow of $p$ coincides with the first arrow in $q$ and $p$ is a subpath of $q$. In other words, $q=p t$ for some path $t$ of $\mathcal{Q}$. We have the following lemma.

Lemma 1.3.26. Let $\Lambda=K \mathcal{Q} / I$ be the Brauer configuration algebra associated to a Brauer configuration $\Gamma=\left(\Gamma_{0}, \Gamma_{1}, \mu, \mathfrak{o}\right)$. For $\alpha \in \Gamma_{0} \backslash \mathscr{T}_{\Gamma}$ let $C$ be an element from $\mathscr{C} \mathscr{C}_{(\alpha)}$ and let $p$ be a path with $l(p) \geq 1$ in $\mathcal{Q}$ and such that first arrow of $p$ coincides with the first arrow in $C$. Then $\bar{p} \neq 0$ if and only if $p$ is a prefix of $C^{\mu(\alpha)}$.

Proof. Let $p$ be a path in $\mathcal{Q}$ such that the first arrow of $p$ coincides with the first arrow of $C$. If $p$ is just one arrow we have nothing to prove. We suposse that $p=a_{1} \cdots a_{r}$ with $r>1$. There are two possible cases:

- There exists a positive integer $s$ such that $p$ is a prefix of $C^{s}(s$ could be greater than $\mu(\alpha))$.

- There is $1 \leq i<r$ such that $a_{i} a_{i+1}$ is not a subpath of $C$.

If there is $1 \leq i<r$ such that $a_{i} a_{i+1}$ is not a subpath of $C$, then $a_{i} a_{i+1}$ cannot be a subpath of any special cycle by Proposition 1.3.25, then necessarily $a_{i} a_{i+1}$ is a type three relation and $\bar{p}=0$.

If there exists a positive integer $s$ such that $p$ is a prefix of $C^{\mu(\alpha)}$ we have again two possible cases:

$$
\begin{aligned}
& \text { - } l(p) \leq l\left(C^{\mu(\alpha)}\right) ; \\
& \text { - } l(p)>l\left(C^{\mu(\alpha)}\right) .
\end{aligned}
$$

If we have that $l(p)>l\left(C^{\mu(\alpha)}\right)$ then necessarily $p$ contains $C^{\mu(\alpha)} a_{1}$ which is a type two relation, and hence $\bar{p}=0$.

Now suppose that $l(p) \leq l\left(C^{\mu(\alpha)}\right)$, then we have that $p$ contains no relations of type two or three. By length assumption, type one relations do not affect $o$ and hence $\bar{p} \neq 0$.

As usual, we let $J$ denote the two sided ideal in $K \mathcal{Q}$ generated by all the arrows in $\mathcal{Q}$. Recall we say that an ideal of $I$ in $K \mathcal{Q}$ is admissible if there exists an integer $N \geq 2$ such that $J^{N} \subset I \subset J^{2}$. We know that if $I$ is an admissible ideal then $\Lambda$ must be finite dimensional. 
Proposition 1.3.27. Let $\Lambda=K \mathcal{Q} / I$ be the Brauer configuration algebra associated to a Brauer configuration $\Gamma=\left(\Gamma_{0}, \Gamma_{1}, \mu, \mathfrak{o}\right)$. Then the ideal $I$ is admissible and $\Lambda$ is a symmetric algebra.

Proof. From the definition of the relations of the generating set for $I$ we see that $I \subset J^{2}$. Let $\mathscr{S}$ the subset of $K \mathcal{Q}$ defined by

$$
\mathscr{S}=\bigcup_{\alpha \in \Gamma_{0} \backslash \mathscr{T}_{\Gamma}}\left\{C^{\mu(\alpha)} \mid C \in \mathscr{C} \mathscr{C}_{(\alpha)}\right\}
$$

Let $N=\max \{l(D) \mid D \in \mathscr{S}\}+1$. By the bijection of the function $f$ defined in page 19 we see that every arrow in $\mathcal{Q}$ is the prefix of some cycle in $\mathscr{C} \mathscr{C}$ and hence in $\mathscr{S}$. Now, if $p$ is a cycle of length $N$ then $p$ cannot be a prefix of any element in $\mathscr{S}$. Using these observations and Lemma 1.3.26 we can say that $\bar{p}=0$, that is, $p \in I$. So we have $J^{N} \subset I$ and it follows that $I$ is admissible.

Using Lema 1.3.26 can be checked that the two sided socle of $\Lambda$ is generated by the elements of $\mathscr{S}$. In fact, we can say that the subset of $\Lambda$ given by $\left\{C^{(V)} \mid V \in \Gamma_{1}\right\}$, and where $C^{(V)}$ was defined in (1.19), forms a $K$-basis of the two sided socle of $\Lambda$.

Now let $\phi: \Lambda \rightarrow K$ be the $K$-linear form defined as follows: let $p$ be a path in $\mathcal{Q}$, then

$$
\phi(\bar{p})= \begin{cases}1, & p \in \mathscr{S} \\ 0, & p \notin \mathscr{S}\end{cases}
$$

Again by Lemma 1.3.26 we can affirm that each element of the $K$-basis of $\Lambda$ is a prefix of some element in $\mathscr{S}$, then is easy to show that the $K$-linear form $\phi$ satisfies $\phi(a b)=\phi(b a)$. Let $\mathfrak{I}$ be a nonzero left ideal contained in $\operatorname{ker} \phi$ and let $z$ be a path in $\mathcal{Q}$ such that $\bar{z}$ is a member of the $K$-basis of $\mathfrak{I}$. So we have that $z$ must be a prefix of some element in $\mathscr{S}$, then there exist a path $y$ in $\mathcal{Q}$ and a polygon $V$ in $\Gamma_{1}$ such that $\bar{y} \bar{z}=C^{(V)} \in \mathfrak{I}$, i.e, $\phi(\bar{y} \bar{z})=\phi\left(C^{(\alpha)}\right)=1$ but this is not possible because $\mathfrak{I} \subset \operatorname{ker} \phi$. In analogous way we can also prove that $\operatorname{ker} \phi$ contains no nonzero right ideals. It follows that $\Lambda$ is a symmetric algebra.

For $\alpha \in \Gamma_{0} \backslash \mathscr{T}_{\Gamma}$ and $C \in \mathscr{C}_{(\alpha)}$ let $\mathscr{P}_{r e}(C)$ denote the collection of all paths in $\mathcal{Q}$ that are prefix of $C$. It is clear that $|\mathscr{P} r e(C)|=\operatorname{val}(\alpha)$. The next result provides a useful $K$-basis of $\Lambda$.

Proposition 1.3.28. Let $\Lambda$ be the Brauer configuration algebra associated to the Brauer configuration $\Gamma=\left(\Gamma_{0}, \Gamma_{1}, \mu, \mathfrak{o}\right)$. Then the collection given by

$$
\left(\bigcup_{\alpha \in \Gamma_{0} \backslash \mathscr{T}_{\Gamma}}\left\{\bar{C}^{j} \bar{q} \mid C \in \mathscr{C}_{\mathscr{C}}(\alpha), q \in \mathscr{P} r e(C), 0 \leq j<\mu(\alpha)\right\}\right) \cup\left\{C^{(V)} \mid V \in \Gamma_{1}\right\}
$$

is a $K$-basis of $\Lambda$.

Proof. We mentioned in the proof of Proposition 1.3.27 that $\left\{C^{(V)} \mid V \in \Gamma_{1}\right\}$ is a $K$-basis of the socle of $\Lambda$. We know that every arrow is the start of a special cycle, so using Lemma 1.3.26 and that the only relations affecting proper subpaths of special cycles are both type one and type three relations, the result follows. 


\subsubsection{Decomposable and indecomposable Brauer configuration al- gebras}

In this subsection we are going to give sufficient and necessary conditions about the decomposability of a Brauer configuration algebra through its Brauer configuration. We start by observing disconnectedness of Brauer configurations.

Proposition 1.3.29. Let $\Gamma$ be a Brauer configuration and suppose that decomposes into Brauer configurations $\Gamma^{\prime} \cup \Gamma^{\prime \prime}$. Then the associated Brauer configuration algebra $\Lambda_{\Gamma}$ is isomorphic to the product $\Lambda_{\Gamma^{\prime}} \times \Lambda_{\Gamma^{\prime \prime}}$.

Proof. By the very same Definition 1.3.5 about disconnected Brauer configurations given in Subsection 1.3.1, we can say that there can be no arrows between vertices in the quivers $\mathcal{Q}_{\Gamma^{\prime}}$ and $\mathcal{Q}_{\Gamma^{\prime \prime}}$. So the result follows.

The next result is the converse of the previuos proposition.

Proposition 1.3.30. If the Brauer configuration $\Gamma$ is connected then the Brauer configuration algebra associated to $\Gamma$ is indecomposable as an algebra.

Proof. We show that if the Brauer configuration algebra $\Lambda$ is decomposable then its Brauer configuration $\Gamma$ is disconnected. To do this we first show that in this case $\Gamma_{0}$ and $\Gamma_{1}$ each admits a partition. Assume that $\Lambda$ is decomposable and that $\Lambda \cong \Lambda^{\prime} \times \Lambda^{\prime \prime}$. Let $\mathcal{Q}, \mathcal{Q}^{\prime}$ and $\mathcal{Q}^{\prime \prime}$ be the quivers of $\Lambda, \Lambda^{\prime}$ and $\Lambda^{\prime \prime}$ respectively. So we have that $\mathcal{Q}$ is the disjoint union of the quivers $\mathcal{Q}^{\prime}$ and $\mathcal{Q}^{\prime \prime}$. Let $\mathfrak{A}$ and $\mathfrak{B}$ be the collections defined by

$$
\begin{aligned}
\mathfrak{A} & =\left\{V \in \Gamma_{1} \mid \text { the vertex in } \mathcal{Q} \text { associated to } V \text { is in } \mathcal{Q}^{\prime}\right\} \\
\mathfrak{B} & =\left\{V \in \Gamma_{1} \mid \text { the vertex in } \mathcal{Q} \text { associated to } V \text { is in } \mathcal{Q}^{\prime \prime}\right\}
\end{aligned}
$$

Because $\mathcal{Q}$ is the disjoint union of $\mathcal{Q}^{\prime}$ and $\mathcal{Q}^{\prime \prime}$ is clear that $\mathfrak{A} \cup \mathfrak{B}=\Gamma_{1}$ and $\mathfrak{A} \cap \mathfrak{B}=\emptyset$.

Let $\mathscr{A}$ be the set of vertices of the polygons in $\mathfrak{A}$ and $\mathscr{B}$ be the set of vertices of the polygons in $\mathfrak{B}$. If $\mathscr{A} \cap \mathscr{B} \neq \emptyset$ then there is a vertex $\alpha \in \Gamma_{0}$ such that $\alpha \in V$ and $\alpha \in V^{\prime}$ for polygons $V \in \mathfrak{A}$ and $V^{\prime} \in \mathfrak{B}$. Then both $V$ and $V^{\prime}$ occur in the successor sequence at $\alpha$. So if $C$ is a special $\alpha$-cycle, both $v$ and $v^{\prime}$ occur as vertices in $C$. But this contradicts the fact that there are no paths from $v$ to $v^{\prime}$ in the quiver of $\Lambda$ because $v$ is a vertex in $\mathcal{Q}^{\prime}$ and $v^{\prime}$ is a vertex in $\mathcal{Q}^{\prime \prime}$. Then we have $\mathscr{A} \cap \mathscr{B}=\emptyset$. Because the condition $\mathrm{C} 1$ of $\Gamma$ it follows that $\Gamma_{0}=\mathscr{A} \cup \mathscr{B}$ is a partition.

So, until now we have that $\Lambda$ decomposable implies that $\Gamma_{0}$ and $\Gamma_{1}$ can be both partitioned. We suppose that $\Gamma$ is connected. From Definition 1.3 .5 we see that necessarily $\Gamma$ does not satisfy the condition (3), then there is a polygon $V$ and vertices $\beta \in \mathscr{A}$ and $\alpha \in$ mathscr $B$ such that $\alpha$ and $\beta$ are vertices of $V$. Then any $\alpha$-cycle has its vertices in $\mathcal{Q}^{\prime \prime}$ and any $\beta$-cycle has its vertices in $\mathcal{Q}^{\prime}$, but $\alpha$ and $\beta$ are vertices of $V$ which is no possible because $\mathfrak{A} \cap \mathfrak{B}=\emptyset$.

\subsection{Induced boundary maps on the Hochschild complex}

Let $\Lambda$ be a finite dimensional algebra (not necessarily a Brauer configuration algebra), and let $\Lambda^{e}=\Lambda^{\mathrm{op}} \otimes_{K} \Lambda$ be the enveloping algebra of $\Lambda$. All the tensor products will be 
on the field $K$. The computation of the Hochschild cohomology begins with constructing a $\Lambda^{e}$-projective resolution of $\Lambda$

$$
P: \cdots P_{n+1} \stackrel{d_{n+1}}{\longrightarrow} P_{n} \stackrel{d_{n}}{\longrightarrow} \cdots \stackrel{d_{2}}{\longrightarrow} P_{1} \stackrel{d_{1}}{\longrightarrow} P_{0} \stackrel{\pi}{\longrightarrow} \Lambda \longrightarrow 0
$$

Here each $P_{i}$ is a right $\Lambda^{e}$-module and $\operatorname{Im} d_{i+1}=\operatorname{ker} d_{i}$ for all $i \geq 0$. Applying the contravariant functor $\operatorname{Hom}_{\Lambda^{e}}(-, \Lambda)$ to $P$ in $(1.20)$ we obtain the following complex

$$
0 \longrightarrow \operatorname{Hom}_{\Lambda^{e}}\left(P_{0}, \Lambda\right) \stackrel{\widehat{d}_{1}}{\longrightarrow} \operatorname{Hom}_{\Lambda^{e}}\left(P_{1}, \Lambda\right) \stackrel{\widehat{d}_{2}}{\longrightarrow} \cdots \stackrel{\widehat{d}_{n}}{\longrightarrow} \operatorname{Hom}_{\Lambda^{e}}\left(P_{n}, \Lambda\right) \stackrel{\widehat{d}_{n+1}}{\longrightarrow} \cdots
$$

where each $\widehat{d}_{i}$ is the boundary map induced by $d_{i}$ for all $i \geq 1$. That is, if $f \in \operatorname{Hom}_{\Lambda^{e}}\left(P_{i-1}, \Lambda\right)$ then $\widehat{d}_{i}(f)=f d_{i}$. From this complex the $i$-th Hochschild cohomology group of $\Lambda$ is defined by $\operatorname{HH}^{i}(\Lambda)=\operatorname{ker} \widehat{d}_{i+1} / \operatorname{im} \widehat{d}_{i}$. From this definition we see that does not depend on the $\Lambda^{e}$ projective resolution given in (1.20). We also see that $\operatorname{HH}^{i}(\Lambda)=\operatorname{Ext}_{\Lambda^{e}}^{i}(\Lambda, \Lambda)$.

We are not interested, at the moment, in a complete $\Lambda^{e}$-projective resolution of $\Lambda$. In fact, we are interested in just a small segment of a particular minimal $\Lambda^{e}$-projective resolution of $\Lambda$, where $\Lambda=K \mathcal{Q} / I$ with $\mathcal{Q}$ a finite quiver and $I$ an admissible ideal of $K \mathcal{Q}$. For this we use one of the constructions in [GS04].

Remember that if $\pi: K \mathcal{Q} \rightarrow \Lambda$ is the canonical surjection we denote by $\bar{x}$ the image of an element $x \in K \mathcal{Q}$ under the morphism $\pi$.

Let's consider the following segment of the minimal $\Lambda^{e}$-projective resolution of $\Lambda=k \mathcal{Q} / I$

$$
P_{1} \stackrel{d_{1}}{\longrightarrow} P_{0} \stackrel{g}{\longrightarrow} \Lambda \longrightarrow 0
$$

where the $\Lambda$-bimodules $P_{0}, P_{1}$ are given by

$$
\begin{aligned}
& P_{0}=\coprod_{v \in \mathcal{Q}_{0}} \Lambda v \otimes v \Lambda, \\
& P_{1}=\coprod_{a \in \mathcal{Q}_{1}} \Lambda s(a) \otimes t(a) \Lambda,
\end{aligned}
$$

and the $\Lambda$-bimodule homomorphisms $g$ and $d_{1}$ are defined by

$$
\begin{gathered}
P_{0} \quad \stackrel{g}{\longrightarrow} \Lambda \\
v \otimes_{v} v \stackrel{\longmapsto}{\longmapsto}, \\
P_{1} \stackrel{d_{1}}{\longrightarrow} s(a) \otimes_{s(a)} \bar{a}-\bar{a} \otimes_{t(a)} t(a) \\
s(a) \otimes_{a} t(a) \stackrel{P_{0}}{\longmapsto} s(.)
\end{gathered}
$$

Here $v \otimes_{v} v$ means the element in $P_{0}$ that has all its entries equal to zero except the $v$-entry which is equal to $v \otimes v$. Means the same for $P_{1}$. That is, $s(a) \otimes_{a} t(a)$ is the element in $P_{1}$ that has all its entries equal to zero except the $a$-entry which is equal to $s(a) \otimes t(a)$. Applying the contravariant functor $\operatorname{Hom}_{\Lambda^{e}}(-, \Lambda)$ to the complex in $(1.21)$ we obtain the complex

$$
0 \longrightarrow \operatorname{Hom}_{\Lambda^{e}}\left(P_{0}, \Lambda\right) \stackrel{\widehat{d}_{1}}{\longrightarrow} \operatorname{Hom}_{\Lambda^{e}}\left(P_{1}, \Lambda\right) \text {. }
$$

It is well known that $\operatorname{HH}^{0}(\Lambda)=\operatorname{ker} \widehat{d}_{1}=Z(\Lambda)$, and where $Z(\Lambda)$ denotes the center of $\Lambda$. We are not going to make the computation of ker $\widehat{d_{1}}$ directly from the homomorphism $\widehat{d_{1}}$. We use the ideas exposed in [Bar01] and [BM98]. So we need first to make some definitions: for 
$v \in \mathcal{Q}_{0}$ and $\lambda \in v \Lambda v$ we define the $\Lambda$-bimodule homomorphism $m_{\lambda}: \Lambda v \otimes v \Lambda \rightarrow \Lambda$ given by

$$
\begin{array}{ccc}
\Lambda v \otimes v \Lambda & \stackrel{m_{\lambda}}{\longrightarrow} \quad \Lambda \\
\alpha \otimes \beta & \longmapsto & \alpha \lambda \beta
\end{array} .
$$

And for $w \in \mathcal{Q}_{0}$ we will denote by $\pi_{w}: \coprod_{v \in \mathcal{Q}_{0}} \Lambda v \otimes v \Lambda \rightarrow \Lambda w \otimes w \Lambda$ the natural projection onto the component of $w$. By Lemma 1.1.6 there exist natural isomorphisms of vector spaces

$$
\begin{gathered}
\operatorname{Hom}_{\Lambda^{e}}\left(P_{0}, \Lambda\right) \cong \coprod_{v \in \mathcal{Q}_{0}} v \Lambda v, \\
\operatorname{Hom}_{\Lambda^{e}}\left(P_{1}, \Lambda\right) \cong \coprod_{a \in \mathcal{Q}_{1}} s(a) \Lambda t(a) .
\end{gathered}
$$

If we take $\lambda \in v \Lambda v$ and denote by $\tilde{\lambda}$ the element in $\coprod_{v \in \mathcal{Q}_{0}} v \Lambda v$ such that all its entries are equal to zero except the entry at $v$ which is equal to $\lambda$, then we can define a $\Lambda$-bimodule homomorphism $d_{1}^{*}: \coprod_{v \in \mathcal{Q}_{0}} v \Lambda v \rightarrow \coprod_{a \in \mathcal{Q}_{1}} s(a) \Lambda t(a)$ given by

$$
\begin{aligned}
& \coprod_{v \in \mathcal{Q}_{0}} v \Lambda v \longrightarrow d_{1}^{*} \longrightarrow \coprod_{a \in \mathcal{Q}_{1}} s(a) \Lambda t(a) \\
& \quad \tilde{\lambda} \longmapsto\left(m_{\lambda}\left(\pi_{v}\left(d_{1}\left(s(a) \otimes_{a} t(a)\right)\right)\right)\right)_{a \in \mathcal{Q}_{1}}
\end{aligned}
$$

Because the way that the $\Lambda$-bimodule homomorphism $d_{1}^{*}$ is defined we can prove that the following diagram

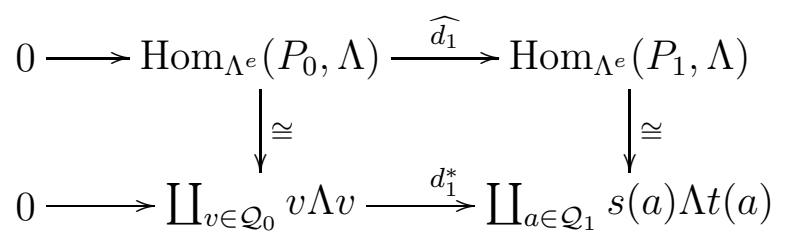

is commutative, where the vertical homomorphisms are the natural isomorphisms mentioned earlier. So by the commutativity of (1.24) we see that we can work with the $\Lambda$-bimodule homomorphism $d_{1}^{*}$ instead of $\widehat{d}_{1}$ to compute $Z(\Lambda)$.

We can give a better expression for the image under $d_{1}^{*}$ of an arbitrary element in the domain. Let $v$ be a vertex in the quiver $\mathcal{Q}, R_{0}=\coprod_{v \in \mathcal{Q}_{0}} v \Lambda v$ and $\pi_{v}^{\prime}: R_{0} \rightarrow v \Lambda v$ be the respective natural projection onto the component $v$. For $\chi \in R_{0}$ we denote by $\chi_{v}$ the element defined by the expression

$$
\chi_{v}:=\pi_{v}^{\prime}(\chi),
$$

and by $\widetilde{\chi_{v}}$ the element in $R_{0}$ such that has all its entries equal to zero except the $v$-entry which is equal to $\chi_{v}$. So from this notation we have that

$$
\chi=\sum_{v \in \mathcal{Q}_{0}} \widetilde{\chi_{v}} .
$$


Using (1.23) to calculate $d_{1}^{*}(\chi)$ we obtain

$$
\begin{aligned}
d_{1}^{*}(\chi) & =\sum_{v \in \mathcal{Q}_{0}} d_{1}^{*}\left(\widetilde{\chi_{v}}\right), \\
& =\sum_{v \in \mathcal{Q}_{0}}\left(m_{\chi_{v}}\left(\pi_{v}\left(s(a) \otimes_{s(a)} \bar{a}\right)\right)\right)_{a \in \mathcal{Q}_{0}}-\sum_{v \in \mathcal{Q}_{0}}\left(m_{\chi_{v}}\left(\pi_{v}\left(\bar{a} \otimes_{t(a)} t(a)\right)\right)\right)_{a \in \mathcal{Q}_{0}}, \\
& =\left(\chi_{s(a)} \bar{a}-\bar{a} \chi_{t(a)}\right)_{a \in \mathcal{Q}_{1}} .
\end{aligned}
$$

That is, for any $\chi \in R_{0}$

$$
d_{1}^{*}(\chi)=\left(\chi_{s(a)} \bar{a}-\bar{a} \chi_{t(a)}\right)_{a \in \mathcal{Q}_{1}} .
$$




\section{Chapter 2}

\section{Center and Dimension}

\section{$2.1 \quad$ Basis of $v \Lambda v$}

Let $\Gamma$ be a Brauer configuration, $\Lambda$ the induced Brauer configuration algebra and let $\mathcal{Q}$ be the induced quiver by $\Gamma$. Using the induced boundary maps to compute the center of $\Lambda$, we need first to calculate a $K$-basis of the space $v \Lambda v$, for every vertex $v$ in $\mathcal{Q}$. To obtain this $K$-basis we need to consider other type of characteristic elements of the associated Brauer configuration algebra different than the already known special cycles. As a starting point we consider the following example.

Example 2.1.1. Let $\Gamma=\left(\Gamma_{0}, \Gamma_{1}, \mu, \mathfrak{o}\right)$ be a Brauer configuration and let $\mathcal{Q}$ be its induced quiver. Suppose that in the configuration $\Gamma$ we have a non-trucated vertex $\alpha$ which has a successor sequence given by

$$
\alpha: V<V<V<V<V<W<W<W<W<W .
$$

We see in this case that $\operatorname{occ}(\alpha, V)=\operatorname{occ}(\alpha, W)=5$. So having in mind this successor sequence we can say that we have the subquiver of $\mathcal{Q}$.

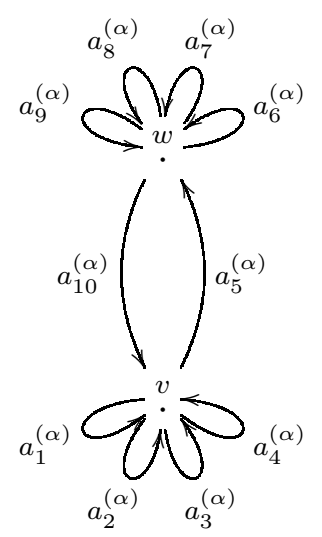

In (2.1) is easy to see the special $\alpha$-cycles at $v$ and $w$. But there are many more elements that are in the space $v \Lambda v$ and they are not special cycles. This is not easily seen from the previous graphical expression. If we use the graphical representation that was introduced in Subsection 1.3.3, in Figure 1.1, to represent (2.1) we obtain the following figure 


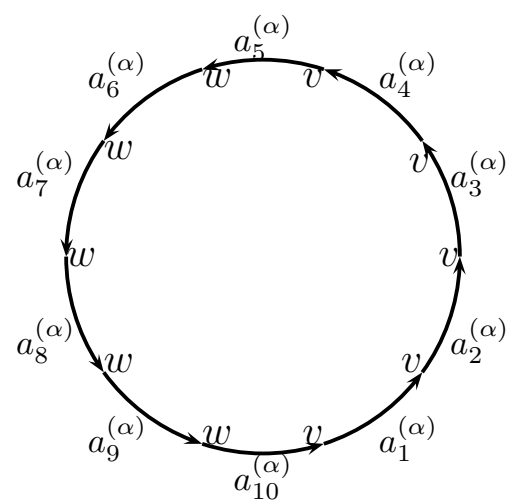

Figure 2.1

If we are only interested to look for oriented cycles associated to $\alpha$ at the vertex $v$ of the quiver, we simply erase the $w$ 's and watch the $v$ 's.

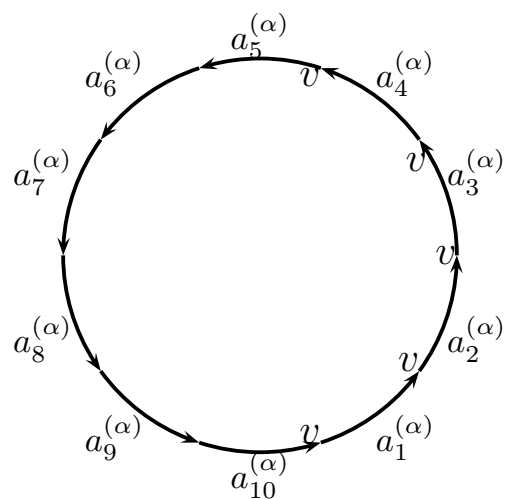

Figure 2.2

Now consider the following elements

$$
\begin{gathered}
q_{1}^{(\alpha, v)}=a_{1}^{(\alpha)}, q_{2}^{(\alpha, v)}=a_{2}^{(\alpha)}, q_{3}^{(\alpha, v)}=a_{3}^{(\alpha)}, \\
q_{4}^{(\alpha, v)}=a_{4}^{(\alpha)}, q_{5}^{(\alpha, v)}=a_{5}^{(\alpha)} a_{6}^{(\alpha)} \cdots a_{10}^{(\alpha)}
\end{gathered}
$$

Each of this elements is an oriented cycle at $v$. By defining $q_{6}^{(\alpha, v)}=q_{1}^{(\alpha, v)}$ and reducing subindices modulo occ $(\alpha, V)=5$, we can see that all elements in the set

$$
\bigcup_{r=1}^{4}\left\{q_{l}^{(\alpha, v)} \cdots q_{l+r-1}^{(\alpha, v)} \mid 1 \leq l \leq 5\right\}
$$

are oriented cycles at $v$ associated to $\alpha$. As this example shows there are many oriented cycles at the vertex $v$ which are not special cycles.

\subsubsection{Non-special cycles}

To apply the idea expressed in Example 2.1.1 we need to improve our notation of the special cycles in the quiver. For $\alpha \in \mathscr{D}_{\Gamma}$ fixed let $\alpha: V_{i_{1}}<\cdots<V_{i_{\mathrm{val}(\alpha)}}$ be the associated 
successor sequence. Then in the quiver $\mathcal{Q}$ we have a sequence of arrows

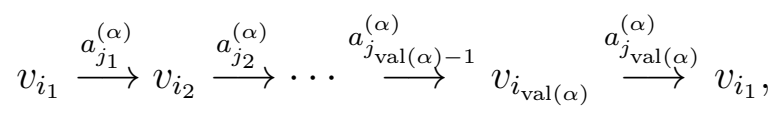

where $v_{i_{k}}$ is the vertex in $\mathcal{Q}$ associated to the polygon $V_{i_{k}}$, for every $1 \leq k \leq \operatorname{val}(\alpha)$. Using Figure 1.1 to represent the sequence of arrows in (2.2) we obtain the following figure.

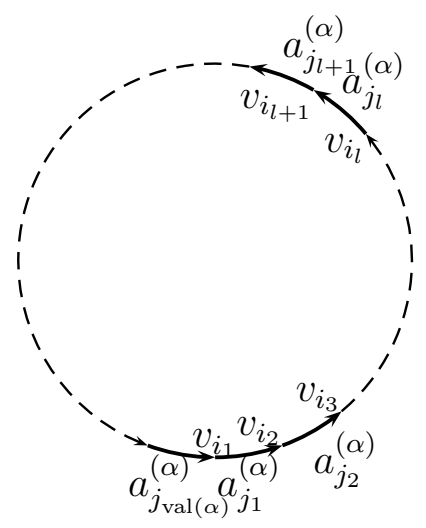

Figure 2.3

Now for a polygon $V \in \Gamma_{1}$ we suppose that $\operatorname{occ}(\alpha, V)>1$ then there are indices $l_{1}<\cdots<$ $l_{\text {occ }(\alpha, V)}$ such that $v=v_{i_{l_{t}}}$, for each $1 \leq t \leq \operatorname{occ}(\alpha, V)$, and where $v$ is the vertex in $\mathcal{Q}$ associated to $V$. From Figure 2.3 we are going to derive another one. So, in this figure we first delete all the vertices that are not equal to $v$. Then we obtain the following.

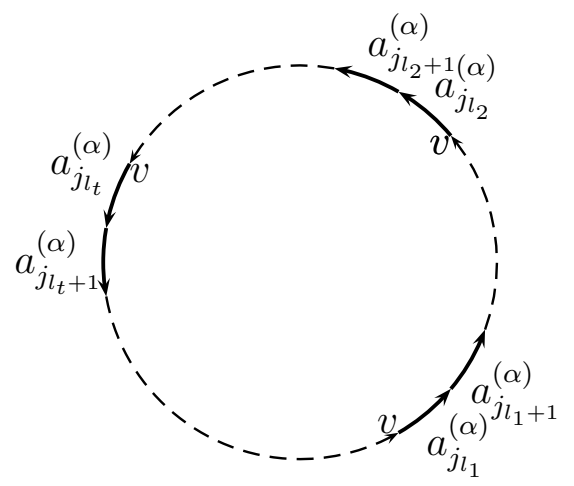

Figure 2.4

Now in Figure 2.4 fix one of the vertices $v$, any vertex you want, and label it as $1 s t v$; in counter clockwise consider the next one $v$ and label it as $2 n d v$, and then the next one and label it as $3 r d v$, and so on. At each occurrence of these labeled $v$ 's we define a new type of cycles in $\mathcal{Q}$.

- At the $1 s t v$ we denote by $C_{1}^{(\alpha, v)}$ the special $\alpha$-cycle at $v$ corresponding to the $1 s t v$, and define $q_{1}^{(\alpha, v)}$ as the oriented cycle obtained by composing all the arrows between the 1 st $v$ and the $2 n d v$. 
- At the 2nd $v$ we denote by $C_{2}^{(\alpha, v)}$ the special $\alpha$-cycle at $v$ corresponding to the $2 n d v$, and define $q_{2}^{(\alpha, v)}$ as the oriented cycle obtained by composing all the arrows between the $2 n d v$ and the $3 r d v$.

- At the $3 r d v$ we denote by $C_{3}^{(\alpha, v)}$ the special $\alpha$-cycle at $v$ corresponding to the $3 r d v$, and define $q_{3}^{(\alpha, v)}$ as the oriented cycle obtained by composing all the arrows between the $3 r d v$ and the 4 th $v$.

- And so on ,...

After repeating this procedure a finite number of times we obtain the following figure.

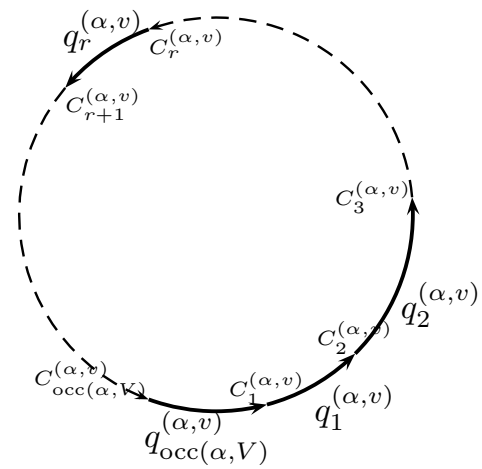

Figure 2.5

We define the non-special $\alpha$-cycles at $v$ to be the collection of oriented cycles $q_{1}^{(\alpha, v)}, \ldots, q_{\mathrm{occ}(\alpha, V)}^{(\alpha, v)}$ in $\mathcal{Q}$. We denote this collection by $\neg \mathscr{C}_{(\alpha)}^{v}$. From the same construction we see that $\left|\mathscr{C}_{(\alpha)}^{v}\right|=$ $\left|\neg \mathscr{C}_{(\alpha)}^{v}\right|=\operatorname{occ}(\alpha, V)$. By defining $q_{\mathrm{occ}(\alpha, V)+1}^{(\alpha, v)}=q_{1}^{(\alpha, v)}$ and reducing subindices modulo occ $(\alpha, V)$, we see that all compositions of the form $q_{l}^{(\alpha, v)} \cdots q_{l+k-1}^{(\alpha, v)}$ are oriented cycles in $\mathcal{Q}$ with initial and final vertex $v$, for every $1 \leq l \leq \operatorname{occ}(\alpha, V)$ and every $1 \leq k \leq \operatorname{occ}(\alpha, V)-1$. When $\operatorname{occ}(\alpha, V)=1$ we set $\neg \mathscr{C}_{(\alpha)}^{v}=\emptyset$. We have the following properties.

Proposition 2.1.2. Let $\Gamma$ be a Brauer configuration with induced quiver $\mathcal{Q}$ and $\alpha \in \mathscr{D}_{\Gamma}$. Let $V \in \mathscr{V}_{(\alpha)}$ such that $\operatorname{occ}(\alpha, V)>1$, and $v$ be the vertex in $\mathcal{Q}$ associated to the polygon $V$. Then reducing all subindices modulo occ $(\alpha, V)$ we have

1. $C_{j}^{(\alpha, v)}=q_{j}^{(\alpha, v)} \cdots q_{j+\operatorname{occ}(\alpha, V)-1}^{(\alpha, v)}, \forall 1 \leq j \leq \operatorname{occ}(\alpha, V)$.

2. $\left(C_{j}^{(\alpha, v)}\right)^{l} q_{j}^{(\alpha, v)}=q_{j}^{(\alpha, v)}\left(C_{j+1}^{(\alpha, v)}\right)^{l}, \forall l>0 ; \forall 1 \leq j \leq \operatorname{occ}(\alpha, V)$.

3. $\bigcup_{k=1}^{\text {occ }(\alpha, V)-1}\left\{q_{l}^{(\alpha, v)} \cdots q_{l+k-1}^{(\alpha, v)} \mid 1 \leq l \leq \operatorname{occ}(\alpha, V)\right\} \subset v \mathcal{Q} v$, where $v \mathcal{Q} v$ is the collection of all the oriented cycles in $\mathcal{Q}$ with initial and final vertex $v$.

Proof. It follows using Figure 2.5.

The collection $\mathscr{D}_{\Gamma}$ defined in (1.6) admits a natural partition that depends entirely on the multiplicity function of the configuration. So we define the following subsets of vertices of the configuration given by

$$
\begin{aligned}
& \mathscr{A}_{\Gamma}=\left\{\alpha \in \mathscr{D}_{\Gamma} \mid \mu(\alpha)>1\right\} \\
& \mathscr{B}_{\Gamma}=\left\{\beta \in \mathscr{D}_{\Gamma} \mid \mu(\beta)=1\right\} .
\end{aligned}
$$


Is clear that $\mathscr{D}_{\Gamma}=\mathscr{A}_{\Gamma} \cup \mathscr{B}_{\Gamma}$ and $\Gamma_{0} \backslash \mathscr{T}_{\Gamma}=\mathscr{A}_{\Gamma} \dot{\cup} \mathscr{B}_{\Gamma} \dot{\cup} \mathscr{C}_{\Gamma}$, where $\mathscr{C}_{\Gamma}$ is defined in (1.5).

Proposition 2.1.3. Let $\Lambda$ be the Brauer configuration algebra induced by $\Gamma$. Then for any $V \in \Gamma_{1}$

$$
\operatorname{dim}_{K} v \Lambda v=2+\sum_{\alpha \in V \cap \Gamma_{0}} \operatorname{occ}(\alpha, V)(\operatorname{occ}(\alpha, V) \mu(\alpha)-1)
$$

where $v$ is the vertex in the induced quiver $\mathcal{Q}$ associated to $V$.

Proof. First we define a collection of sets associated to each polygon of the configuration. For the polygon $V \in \Gamma_{1}$ define the following sets of vertices

$$
\begin{aligned}
\hat{V} & =\left(V \cap \mathscr{A}_{\Gamma}\right) \cup\left(V \cap \mathscr{C}_{\Gamma}\right), \\
\mathscr{A}_{\Gamma}^{V>1} & =\left\{\alpha \in V \cap \mathscr{A}_{\Gamma} \mid \operatorname{occ}(\alpha, V)>1\right\}, \\
\mathscr{B}_{\Gamma}^{V>1} & =\left\{\beta \in V \cap \mathscr{B}_{\Gamma} \mid \operatorname{occ}(\beta, V)>1\right\} .
\end{aligned}
$$

Now in the induced Brauer configuration algebra $\Lambda$ define the following subsets of the $K$ space $v \Lambda v$

$$
\begin{aligned}
& \mathscr{L}_{1}=\bigcup_{\alpha \in \hat{V}}\left\{\bar{C}^{j} \mid C \in \mathscr{C}_{(\alpha)}^{v}, 1 \leq j \leq \mu(\alpha)-1\right\}, \\
& \left.\mathscr{L}_{2}=\bigcup_{\alpha \in \mathscr{A}_{\Gamma}^{V>1}}\left(\bigcup_{k=1}^{\operatorname{occ}(\alpha, V)-1}\left\{\overline{\left(C_{l}^{(\alpha, v)}\right.}\right)^{j} \overline{q_{l}^{(\alpha, v)}} \cdots \overline{q_{l+k-1}^{(\alpha, v)}} \mid 0 \leq j \leq \mu(\alpha)-1,1 \leq l \leq \operatorname{occ}(\alpha, V)\right\}\right), \\
& \mathscr{L}_{3}=\bigcup_{\text {beta } \in \mathscr{B}_{\Gamma}^{V>1}}\left\{\overline{q_{l}^{(\beta, v)}} \cdots \overline{q_{l+k-1}^{(\beta, v)}} \mid 1 \leq l \leq \operatorname{occ}(\beta, V), 1 \leq k \leq \operatorname{occ}(\beta, V)-1\right\} .
\end{aligned}
$$

By Lemma 1.3.26, Proposition 1.3.28,Figure 2.5 and the definition of the non-special cycles, the set $\mathscr{L}_{1} \cup \mathscr{L}_{2} \cup \mathscr{L}_{3} \cup\left\{\bar{v}, C^{(V)}\right\}$ is a $K$-basis of $v \Lambda v$. From the definition of the $\mathscr{L}_{i}$ 's, we see that

$$
\begin{aligned}
\left|\mathscr{L}_{1}\right| & =\sum_{\alpha \in V \cap \mathscr{A}_{\Gamma}} \operatorname{occ}(\alpha, V)(\mu(\alpha)-1)+\sum_{\gamma \in V \cap \mathscr{C}_{\Gamma}}(\mu(\gamma)-1), \\
\left|\mathscr{L}_{2}\right| & =\sum_{\alpha \in \mathscr{A}_{\Gamma}^{V>1}} \operatorname{occ}(\alpha, V)(\operatorname{occ}(\alpha, V)-1) \mu(\alpha), \\
\left|\mathscr{L}_{3}\right| & =\sum_{\beta \in \mathscr{B}_{\Gamma}^{V>1}} \operatorname{occ}(\beta, V)(\operatorname{occ}(\beta, V)-1)
\end{aligned}
$$

but the expressions in (2.9) and (2.10) are respectively equal to

$$
\begin{gathered}
\sum_{\alpha \in V \cap \mathscr{A}_{\Gamma}} \operatorname{occ}(\alpha, V)(\operatorname{occ}(\alpha, V)-1) \mu(\alpha), \\
\sum_{\beta \in V \cap \mathscr{B}_{\Gamma}} \operatorname{occ}(\beta, V)(\operatorname{occ}(\beta, V)-1)
\end{gathered}
$$

Finally using expressions (2.8), (2.11) and (2.12) we obtain

$$
\begin{aligned}
& \operatorname{dim}_{K} v \Lambda v=2+\sum_{\alpha \in V \cap \mathscr{A}_{\Gamma}} \operatorname{occ}(\alpha, V)(\mu(\alpha)-1)+\sum_{\gamma \in V \cap \mathscr{C}_{\Gamma}}(\mu(\gamma)-1)+\sum_{\alpha \in V \cap \mathscr{A}_{\Gamma}} \operatorname{occ}(\alpha, V)(\operatorname{occ}(\alpha, V)-1) \mu(\alpha)+\sum_{\beta \in V \cap \mathscr{B}_{\Gamma}} \operatorname{occ}(\beta, V)(\operatorname{occ}(\beta, V)-1) \\
&=2+\sum_{\alpha \in V \cap \mathscr{A}_{\Gamma}} \operatorname{occ}(\alpha, V)(\operatorname{occ}(\alpha, V) \mu(\alpha)-1)+\sum_{\beta \in V \cap \mathscr{B}_{\Gamma}} \operatorname{occ}(\beta, V)(\operatorname{occ}(\beta, V)-1)+\sum_{\gamma \in V \cap \mathscr{C}_{\Gamma}}(\mu(\gamma)-1)
\end{aligned}
$$




$$
\begin{aligned}
& =2+\sum_{\alpha \in V \cap\left(\mathscr{A}_{\Gamma} \cup \mathscr{B}_{\Gamma} \cup \mathscr{C}_{\Gamma}\right)} \operatorname{occ}(\alpha, V)(\operatorname{occ}(\alpha, V) \mu(\alpha)-1) \\
& =2+\sum_{\alpha \in V \cap\left(\Gamma_{0} \backslash \mathscr{T}_{\Gamma}\right)} \operatorname{occ}(\alpha, V)(\operatorname{occ}(\alpha, V) \mu(\alpha)-1)
\end{aligned}
$$

Now if $\alpha \in V$ is such that $\alpha \in \mathscr{T}_{\Gamma}$ then $\operatorname{val}(\alpha)=\operatorname{occ}(\alpha, V)=1 \operatorname{so} \operatorname{occ}(\alpha, V) \mu(\alpha)=1$, and the expression in (2.13) is equal to

$$
2+\sum_{\alpha \in V \cap \Gamma_{0}} \operatorname{occ}(\alpha, V)(\operatorname{occ}(\alpha, V) \mu(\alpha)-1) .
$$

\subsubsection{Some characteristic elements}

In this subsection we consider some characteristics elements in the Brauer configuration algebra induced by a Brauer configuration.

Let $\Gamma=\left(\Gamma_{0}, \Gamma_{1}, \mu, \mathfrak{o}\right)$ be a Brauer configuration. For $\alpha \in \mathscr{A}_{\Gamma} \cup \mathscr{C}_{\Gamma}$ we denote by $C(\alpha)$ the element in $\Lambda$ defined by

$$
C(\alpha):=\sum_{C \in \mathscr{C} \mathscr{C}_{(\alpha)}} \bar{C}
$$

We refer the reader to the expression in (1.19) to recall the definition of $C^{(V)}$.

Proposition 2.1.4. Let $\Lambda$ be the algebra induced by the Brauer configuration $\Gamma$. Then

1. $\left\{C^{(V)} \mid V \in \Gamma_{1}\right\} \subset \operatorname{soc}_{\Lambda^{e}} \Lambda$.

2. For $\alpha \in \mathscr{A}_{\Gamma} \cup \mathscr{B}_{\Gamma}$ let $C, D \in \mathscr{C} \mathscr{C}_{(\alpha)}$ be special cycles.

(a) If $C \neq D$ then $\bar{C} \bar{D}=\bar{D} \bar{C}=0$.

(b) If $C=D$ and $\alpha \in \mathscr{B}_{\Gamma}$ then $\bar{C}^{2}=0$.

3. For $\alpha \in \mathscr{A}_{\Gamma} \cup \mathscr{C}_{\Gamma}$

$$
C(\alpha)^{\mu(\alpha)}=\sum_{V \in \mathscr{V}_{(\alpha)}} \operatorname{occ}(\alpha, V) C^{(V)} .
$$

4. For any $\alpha \in \Gamma_{0} \backslash \mathscr{T}_{\Gamma}$

$$
\bar{C}^{\mu(\alpha)+1}=0, \forall C \in \mathscr{C}_{(\alpha)} .
$$

Proof. 1. In general we know that if $\mathcal{Q}$ is the quiver of the induced algebra $\Lambda$ then $\operatorname{soc}_{\Lambda^{e}} \Lambda=$ $\left\{x \in \Lambda \mid \bar{a} x=x \bar{a}=0, \forall a \in \mathcal{Q}_{1}\right\}$. So for any $a \in \mathcal{Q}_{1}$ we see that $C^{(V)} \bar{a}$ contains either a relation of type two or a relation or type three, then $C^{(V)} \bar{a}=0$. It happens the same with $\bar{a} C^{(V)}$. We conclude that $C^{(V)} \in \operatorname{soc}_{\Lambda^{e}} \Lambda$, for any $V \in \Gamma_{1}$.

2. If a pair of two distinct elements from $\mathscr{C} \mathscr{C}_{(\alpha)}$ are cycles starting at different vertices, then their respective product in $\Lambda$ must be zero. Now if $C, D \in \mathscr{C}_{(\alpha)}^{v}$ for some $V \in \mathscr{V}_{(\alpha)}$, and $C \neq D$, their product $C D$ must contain a relation of type three then $\bar{C} \bar{D}=0$ in $\Lambda$. We have the same for the product $D C$. If $\alpha \in \mathscr{B}_{\Gamma}$ and $C$ is any cycle in $\mathscr{C} \mathscr{C}_{(\alpha)}$ we have that $C^{2}$ contais a relation of type two, i.e, $\bar{C}^{2}=0$ in $\Lambda$. 
3. Let $\alpha \in \mathscr{A}_{\Gamma} \cup \mathscr{C}_{\Gamma}$. If $\alpha \in \mathscr{C}_{\Gamma}$ then the affirmation holds. If $\alpha \in \mathscr{A}_{\Gamma}$ then we have that for each $V \in \mathscr{V}_{(\alpha)}$

$$
C^{(V)}=\bar{C}^{\mu(\alpha)}, \forall C \in \mathscr{C}_{(\alpha)}^{v} .
$$

So using what we proved in $(2 \mathrm{a})$ and $(2.15)$ it follows that

$$
\begin{aligned}
C(\alpha)^{\mu(\alpha)} & =\sum_{C \in \mathscr{C}_{(\alpha)}} \bar{C}^{\mu(\alpha)} \\
& =\sum_{V \in \mathscr{V}_{(\alpha)}}\left(\sum_{C \in \mathscr{C}_{(\alpha)}^{v}} \bar{C}^{\mu(\alpha)}\right) \\
& =\sum_{V \in \mathscr{V}_{(\alpha)}}\left(\sum_{C \in \mathscr{C}_{(\alpha)}^{v}} C^{(V)}\right) \\
& \left.=\sum_{V \in \mathscr{V}_{(\alpha)}}\left(\sum_{C \in \mathscr{C}_{(\alpha)}^{v}} 1_{K}\right) C^{(V)}\right) \\
& =\sum_{V \in \mathscr{V}_{(\alpha)}} \operatorname{occ}(\alpha, V) C^{(V)} .
\end{aligned}
$$

4. If $\alpha \in \Gamma_{0} \backslash \mathscr{T}_{\Gamma}$ and $C \in \mathscr{C} \mathscr{C}_{(\alpha)}$ the cycle $C^{\mu(\alpha)+1}$ contains a relation of type two then $\bar{C}^{\mu(\alpha)+1}=0$.

Observation 2.1.5. By using Proposition 2.1.4(2) we can show that in general for each $\alpha \in \mathscr{A}_{\Gamma} \cup \mathscr{C}_{\Gamma}$ and each positive integer $j$

$$
\begin{aligned}
C(\alpha)^{j} & =\left(\sum_{C \in \mathscr{C} \mathscr{C}_{(\alpha)}} \bar{C}\right)^{j} \\
& =\sum_{C \in \mathscr{C} \mathscr{C}_{(\alpha)}} \bar{C}^{j}
\end{aligned}
$$

We know that for each $\alpha \in \Gamma_{0} \backslash \mathscr{T}_{\Gamma}$ there is a unique collection of arrows associated to $\alpha$, which is the collection of arrows contained in a special $\alpha$-cycle. Sometimes in this collection appear some loops. These loops induce elements in the center of the induced Brauer configuration algebra.

For $\alpha \in \Gamma_{0} \backslash \mathscr{T}_{\Gamma}$ let $\mathscr{A} \mathscr{A}_{(\alpha)}$ denote the subset of $\mathcal{Q}_{1}$ defined by

$$
\mathscr{A}_{\mathscr{A}}(\alpha):=\left\{a \in \mathcal{Q}_{1} \mid a \text { is contained in a special } \alpha \text {-cycle }\right\} \text {. }
$$

If $\alpha \in \mathscr{C}_{\Gamma}$ we know that $\mathscr{A} \mathscr{A}_{(\alpha)}$ contains only one arrow, which we denote by $a^{(\alpha)}$. Then $\mathscr{A} \mathscr{A}_{(\alpha)}=\left\{a^{(\alpha)}\right\}$. Now, if $\alpha \in \mathscr{D}_{\Gamma}$ then using the notation for arrows introduced in (1.9) we set

$$
\mathscr{A} \mathscr{A}_{(\alpha)}=\left\{a_{1}^{(\alpha)}, \ldots, a_{\mathrm{val}(\alpha)}^{(\alpha)}\right\}
$$

such that $t\left(a_{i}^{(\alpha)}\right)=s\left(a_{i+1}^{(\alpha)}\right)$ for each $1 \leq i \leq \operatorname{val}(\alpha)$, and where $a_{\mathrm{val}(\alpha)+1}^{(\alpha)}=a_{1}^{(\alpha)}$. We call the 
collection $\mathscr{A}_{\mathscr{A}}(\alpha)$ the arrows associated to $\alpha$. Let $h: \mathscr{D}_{\Gamma} \rightarrow\{0,1\}$ be the map defined by

$$
h(\alpha)= \begin{cases}0, & \mathscr{A}_{(\alpha)} \text { contains no loops } \\ 1, & \mathscr{A}_{(\alpha)} \text { contains loops. }\end{cases}
$$

It is easy to check that

$$
\alpha \in h^{-1}(1) \Longleftrightarrow \quad \begin{aligned}
& \exists V \in \mathscr{V}_{(\alpha)} ; \operatorname{occ}(\alpha, V)>1 \text { and } \\
& \exists 1 \leq s \leq \operatorname{occ}(\alpha, V) ; q_{s}^{(\alpha, v)} \in \mathcal{Q}_{1}
\end{aligned}
$$

For $\alpha \in h^{-1}(1)$ let $\mathscr{M}^{(\alpha)}$ be the set of ordered pairs defined by

$$
\mathscr{M}^{(\alpha)}=\{(V, s) \mid V \text { and } s \text { satisfies }(2.19)\} \text {. }
$$

Each pair $(V, s) \in \mathscr{M}^{(\alpha)}$ is associated to a unique loop in $\mathcal{Q}$, the non-special $\alpha$-cycle $q_{s}^{(\alpha, v)}$ in (2.19). For $(V, s) \in \mathscr{M}^{(\alpha)}$ let $D_{V, s}^{(\alpha)}$ be the cycle in $\mathcal{Q}$ defined by

$$
D_{V, s}^{(\alpha)}=\left\{\begin{array}{cc}
\left(C_{s+1}^{(\alpha, v)}\right)^{\mu(\alpha)-1} q_{s+1}^{(\alpha, v)} \cdots q_{s-1}^{(\alpha, v)}, & \alpha \in \mathscr{A}_{\Gamma} ; \\
q_{s+1}^{(\alpha, v)} \cdots q_{s-1}^{(\alpha, v)}, & \alpha \in \mathscr{B}_{\Gamma} .
\end{array}\right.
$$

In (2.21) the composition of cycles $q_{s+1}^{(\alpha, v)} \cdots q_{s-1}^{(\alpha, v)}$ means

$$
q_{s+1}^{(\alpha, v)} \cdots q_{\mathrm{occ}(\alpha, V)}^{(\alpha, v)} q_{1}^{(\alpha, v)} \cdots q_{s-1}^{(\alpha, v)} .
$$

We call $D_{V, s}^{(\alpha)}$ a central mixed $\alpha$-cycle at the vertex $v$ in $\mathcal{Q}$. We denote the collection of these cycles by $\Psi_{(\alpha)}^{v}$, i.e,

$$
\Psi_{(\alpha)}^{v}=\left\{D_{V, s}^{(\alpha)} \mid(V, s) \in \mathscr{M}^{(\alpha)}\right\} .
$$

We should recall that a path $p$ is a prefix of the path $q$ in the quiver $\mathcal{Q}$ if the first arrow of $p$ coincides with the first arrow of $q$ and $p$ is a subpath of $q$. The following lemma is very useful.

Lemma 2.1.6. Let $\Lambda$ be a Brauer configuration algebra associated to the Brauer configuration $\Gamma$. Let $x$ be an element in $\Lambda$ that satisfies the following property

$$
\begin{aligned}
\forall \alpha \in \Gamma_{0} \backslash \mathscr{T}_{\Gamma}, \forall C \in \mathscr{C}^{\mathscr{C}}(\alpha) & \\
\qquad & \text { is a prefix of } C^{\mu(\alpha)} \Longrightarrow \bar{p} x=x \bar{p} .
\end{aligned}
$$

Then $x \in Z(\Lambda)$.

Proof. This follows from Proposition 1.3.28.

The following proposition gives us a couple of collection of elements in the center of a Brauer configuration algebra.

Proposition 2.1.7. Let $\Gamma=\left(\Gamma_{0}, \Gamma_{1}, \mu, \mathfrak{o}\right)$ be a reduced Brauer configuration with associated Brauer configuration algebra $\Lambda$. Then

1.

$$
\bigcup_{\alpha \in \mathscr{C}_{\Gamma}}\left\{\bar{a} \mid a \in \mathscr{A}_{\mathscr{A}_{(\alpha)}}\right\} \subset Z(\Lambda)
$$


2 .

$$
\bigcup_{\alpha \in h^{-1}(1)}\left\{\overline{D_{V, s}^{(\alpha)}} \mid(V, s) \in \mathscr{M}^{(\alpha)}\right\} \subset Z(\Lambda)
$$

Proof. 1. Let $\mathcal{Q}$ be the induced quiver of $\Gamma$. For $\alpha \in \mathscr{C}_{\Gamma}$ let $\beta$ be a nontruncated vertex such that $\beta \neq \alpha$ and let $p$ be a prefix of $C^{\mu(\beta)}$, for some $C \in \mathscr{C} \mathscr{C}_{(\alpha)}$. If $p a^{(\alpha)} \neq 0$ and $a^{(\alpha)} p=0$ in $K \mathcal{Q}$ then $p a^{(\alpha)}$ contains a relation of type three and hence $\overline{p a^{(\alpha)}}=\overline{a^{(\alpha)} p}=0$. In the same way, if $a^{(\alpha)} p \neq 0$ but $p a^{(\alpha)}=0$ then $a^{(\alpha)} p$ also contains a relation of type three and hence $\overline{a^{(\alpha)} p}=\overline{p a^{(\alpha)}}=0$. Now, if $a^{(\alpha)} p \neq 0$ and $p a^{(\alpha)} \neq 0$ then $p$ must be an oriented cycle and hence both $a^{(\alpha)} p$ and $p a^{(\alpha)}$ contain a relation of type three, and also in this case $\overline{a^{(\alpha)} p}=\overline{p a^{(\alpha)}}=0$. Now, if $\beta=\alpha$ then $p$ must be a prefix of $\left(a^{(\alpha)}\right)^{\mu(\alpha)}$ and obviously $p$ commutes with $a^{(\alpha)}$. By Lemma 2.1.6 we can affirm that $a^{(\alpha)} \in Z(\Lambda)$.

2. Let $\alpha \in h^{-1}(1)$ and $(V, s) \in \mathscr{M}^{(\alpha)}$ be an ordered pair that satisfies (2.19). So, we have that the non-special cycle $q_{s}^{(\alpha, v)}$ is a loop and the elements $C_{s}^{(\alpha, v)}, C_{s+1}^{(\alpha, v)}$ and $q_{s}^{(\alpha, v)}$ can be graphically represented as

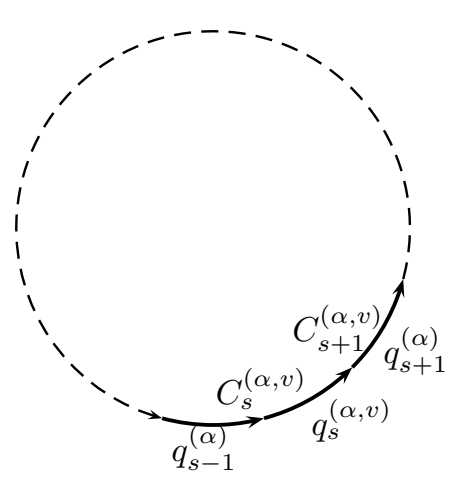

Figure 2.6

Let $D$ be the element in $\Psi_{(\alpha)}^{v}$ associated to the pair $(V, s)$. We will show that $\bar{D}$ is in the center of $\Lambda$. Let $\beta \in \Gamma_{0} \backslash \mathscr{T}_{\Gamma}$ and $p$ be a prefix of $C^{\mu(\beta)}$ for some $C \in \mathscr{C}_{\mathscr{C}}(\beta)$. Suppose that $\alpha \neq \beta$. If $D p \neq 0$ and $p D=0$ in $K \mathcal{Q}$ then the path $D p$ contains a relation of type three, and hence $\overline{D p}=\overline{D p}=0$ in $\Lambda$. In similar way, if $D p=0$ and $p D \neq 0$ in $K \mathcal{Q}$, we also have that $p D$ contains a relation of type three and hence $\overline{p D}=\overline{D p}=0$ in $\Lambda$. If $p D \neq 0$ and $D p \neq 0$ then $p$ must be a cycle, but also in this case both $p D$ and $D p$ contain a relation of type thre, and hence $\overline{p D}=\overline{D p}=0$. Suppose now that $\alpha=\beta$, and without loss of generality assume that $\alpha \in \mathscr{A}_{\Gamma}$ (in the case $\alpha \in \mathscr{B}_{\Gamma}$ the reasonig is analogous). It is sufficient to consider only the case when $p$ is a prefix of $\left(C_{s}^{(\alpha, v)}\right)^{\mu(\alpha)}$. If $\ell(p)>1$ then is easy to see that $D p$ contains a relation of type two, and if $p D \neq 0$ in $K \mathcal{Q}$ then also contains a relation of type two. Hence 
$\overline{D p}=\overline{p D}=0$. If $\ell(p)=1$ then necessarily $p=q_{s}^{(\alpha, v)}$, and by Proposition 2.1.2 we obtain

$$
\begin{aligned}
\bar{D} \bar{p} & =\left(\overline{C_{s+1}^{(\alpha, v)}}\right)^{\mu(\alpha)-1} q_{s+1}^{\overline{(\alpha, v)}} \cdots q_{s-1}^{\overline{(\alpha, v)}} q_{s}^{\overline{(\alpha, v)}} \\
& =\left(\overline{C_{s+1}^{(\alpha, v)}}\right)^{\mu(\alpha)} \\
\bar{p} \bar{D} & =q_{s}^{\overline{(\alpha, v)}}\left(\overline{C_{s+1}^{(\alpha, v)}}\right)^{\mu(\alpha)-1} \overline{q_{s+1}^{(\alpha, v)}} \cdots q_{s-1}^{\overline{(\alpha, v)}} \\
& =\left(\overline{C_{s}^{(\alpha, v)}}\right)^{\mu(\alpha)} .
\end{aligned}
$$

Note that $\left(C_{s}^{(\alpha, v)}\right)^{\mu(\alpha)}-\left(C_{s+1}^{(\alpha, v)}\right)^{\mu(\alpha)}$ is a relation of type three, then (2.23) and (2.24) are equal. By Lemma 2.1.6 we can conclude that $\bar{D}$ is in $Z(\Lambda)$.

Let $\Gamma=\left(\Gamma_{0}, \Gamma_{1}, \mu, \mathfrak{o}\right)$ be a reduced Brauer configuration. From the function $h$ defined in (2.18) let $H$ be the subset of $h^{-1}(1)$ defined as

$$
H=\left\{\alpha \in h^{-1}(1) \mid \operatorname{val}(\alpha)=2 \text { and } \mu(\alpha)=1\right\} .
$$

If $\alpha \in H$ it is easy to see that the collection $\mathscr{A}_{(\alpha)}$ of arrows associated to $\alpha$ only contains loops. If $\mathcal{Q}$ is the induced quiver by $\Gamma$ and $v$ is the vertex in $\mathcal{Q}$ associated to the unique polygon $V$ in $\mathscr{V}_{(\alpha)}$, we have that the quiver

$$
a_{2}^{(\alpha)} v \supset a_{1}^{(\alpha)}
$$

is a subquiver of $\mathcal{Q}$ (see Example 1.3.14). Setting $q_{1}^{(\alpha, v)}=a_{1}^{(\alpha)}$ and $q_{2}^{(\alpha, v)}=a_{2}^{(\alpha)}$ we see that $\mathscr{M}^{(\alpha)}=\{(V, 1),(V, 2)\}$ and

$$
\begin{aligned}
& D_{V, 1}^{(\alpha)}=q_{2}^{(\alpha, v)}=a_{2}^{(\alpha)}, \\
& D_{V, 2}^{(\alpha)}=q_{1}^{(\alpha, v)}=a_{1}^{(\alpha)} .
\end{aligned}
$$

We have the following proposition.

Proposition 2.1.8. Let $\Gamma=\left(\Gamma_{0}, \Gamma_{1}, \mu, \mathfrak{o}\right)$ be a reduced Brauer configuration and let $\Lambda$ be its induced Brauer configuration algebra. If $\alpha \in H$ then

$$
\begin{aligned}
\overline{D_{V, 1}^{(\alpha)}} \overline{D_{V, 2}^{(\alpha)}} & =C^{(V)} \\
\overline{D_{V, i}^{(\alpha)}} & =0, i=1,2 ;
\end{aligned}
$$

with $V$ the only polygon in $\mathscr{V}_{(\alpha)}$.

Proof. If $\alpha \in H$ then we have in particular that $\mu(\alpha)=1$, then using the subquiver in (2.26) the equalities are easily checked.

Example 2.1.9. Let $\Gamma^{(4)}=\left(\Gamma_{0}^{(4)}, \Gamma_{1}^{(4)}, \mu^{(4)}, \mathfrak{o}^{(4)}\right)$ be the Brauer configuration given by

- $\Gamma_{0}^{(4)}=\{1, \alpha, \beta, \gamma\}$

- $\Gamma_{1}^{(4)}=\left\{W_{1}, W_{2}, W_{3}\right\}$ where

$$
\begin{aligned}
& W_{1}=\{1, \alpha, \alpha\}, \\
& W_{2}=\{1, \beta, \beta\}, \\
& W_{3}=\{1, \gamma, \gamma\}
\end{aligned}
$$


- $\mu^{(4)} \equiv 1$

- The orientation $\mathfrak{o}^{(4)}$ induces the following successor sequences

$$
\begin{aligned}
& 1: W_{1}<W_{2}<W_{3} \text {, } \\
& \alpha: W_{1}<W_{1} \\
& \beta: W_{2}<W_{2} \text {, } \\
& \gamma: W_{3}<W_{3} \text {. }
\end{aligned}
$$

If $\mathcal{Q}^{(4)}$ is the induced quiver by $\Gamma^{(4)}$, then this quiver is

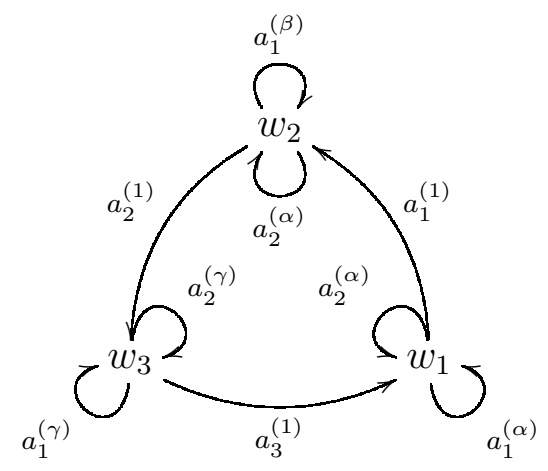

As we can see from this quiver the collection $H$ defined in (2.25) is equal to

$$
H=\{\alpha, \beta, \gamma\}=h^{-1}(1)
$$

\subsection{Dimension of the center of a Brauer configuration algebra}

In the previous section we determined some elements of the center of a Brauer configuration algebra by showing directly that the product of these elements commute with any other element of the algebra. In this section we want to determine all the elements of the center. To do this we use the bimodule homomorphism $d_{1}^{*}$ defined in Section 1.4 (see (1.23)). As we showed there, for an algebra $\Lambda=K \mathcal{Q} / I$ with $\mathcal{Q}$ a finite quiver and $I$ an admissible ideal of $K \mathcal{Q}$, the kernel of the bimodule homomorphism $d_{1}^{*}$ coincides with $Z(\Lambda)$. So our work here will be to compute a $K$-basis of $\operatorname{ker} d_{1}^{*}$ when $\Lambda$ is a Brauer configuration algebra. We start with a preliminary result.

Proposition 2.2.1. Let $\Lambda$ be the Brauer configuration algebra associated to the Brauer configuration $\Gamma$, which is reduced and connected. Assume that $\operatorname{rad}^{2}(\Lambda) \neq 0$. Then for each $a \in \mathcal{Q}_{1}$ there exists a unique $\alpha \in \Gamma_{0} \backslash \mathscr{T}_{\Gamma}$ such that

1. there exist a unique pair $\left(C, C^{\prime}\right)$ of elements in $\mathscr{C} \mathscr{C}_{(\alpha)}$ such that

(a) $C^{l} a=a C^{\prime l}$, for any positive integer $l$.

(b) If $\alpha \notin \mathscr{B}_{\Gamma}$ then $\bar{C} \bar{a} \neq 0$ and $\bar{a} \overline{C^{\prime}} \neq 0$.

2. If $\alpha \in \mathscr{B}_{\Gamma}$ then $\bar{D} \bar{a}=\bar{a} \bar{E}=0$, for any $D, E \in \mathscr{C} \mathscr{C}$.

Proof. 1. Let $a$ be an arrow of the quiver $\mathcal{Q}$. By Proposition 1.3.23(1) there exists a unique $\alpha \in \Gamma_{0} \backslash \mathscr{T}_{\Gamma}$ such that the arrow $a$ appears exactly once in each cycle in $\mathscr{C}_{\mathscr{C}_{(\alpha)}}$. If $\alpha \in \mathscr{C}_{\Gamma}$ then 
$a=a^{(\alpha)}$ is a loop, hence $a$ itself is the unique element in $\mathscr{C} \mathscr{C}(\alpha)$ and $\bar{a}^{2} \neq 0$ in $\Lambda$, and trivially we have the properties hold. If $\alpha \in \mathscr{D}_{\Gamma}$ suppose that $a=a_{i}^{(\alpha)}$, for some $1 \leq i \leq \operatorname{val}(\alpha)$. Then the special $\alpha$-cycles are given respectively by

$$
\begin{aligned}
C & =a_{i}^{(\alpha)} \cdots a_{i-1}^{(\alpha)}, \\
C^{\prime} & =a_{i+1}^{(\alpha)} \cdots a_{i}^{(\alpha)} .
\end{aligned}
$$

Then is not difficult to see that $C^{l} a=a C^{\prime l}$ for any positive integer $l$. The paths $C a$ and $a C^{\prime}$ are prefix of $C^{\mu(\alpha)}$ and $C^{\prime \mu(\alpha)}$, respectively. If $\alpha \notin \mathscr{B}_{\Gamma}$ then by Lemma 1.3.26 we have that $\bar{C} \bar{a} \neq 0$ and $\bar{a} \overline{C^{\prime}} \neq 0$.

2. For the arrow $a$ suppose that $\alpha$, its associated vertex, is in $\mathscr{B}_{\Gamma}$. Let $D, E$ be special cycles such that $t(D)=s(a)$ and $t(a)=s(E)$. The paths $D a$ and $a E$ contain either a relation of type three or, because of $\alpha \in \mathscr{B}_{\Gamma}$, a relation of type two, but any of this situations implies that $\overline{D a}=\overline{a E}=0$ in $\Lambda$.

Let $\Lambda$ be the Brauer configuration algebra associated to the Brauer configuration $\Gamma$ which is reduced and connected, and assume that $\operatorname{rad}^{2}(\Lambda) \neq 0$. Let $R_{0}=\coprod_{v \in \mathcal{Q}_{0}} v \Lambda v$ and $\pi_{v}^{\prime}: R_{0} \rightarrow v \Lambda v$ be the natural projection onto $v \Lambda v$. Let $\chi$ be an arbitrary element of the $K$-space $R_{0}$. We can express $\chi$ as

$$
\chi=\sum_{v \in \mathcal{Q}_{0}} \widetilde{\chi_{v}},
$$

where $\widetilde{\chi_{v}}$ is the element in $R_{0}$ such that has all its entries equal to zero except the $v$-entry, that is, $\chi_{v}=\pi_{v}^{\prime}(\chi)$. By Proposition 2.1.3 and its proof we have that the element $\chi_{v}$ of $v \Lambda v$ can be expressed by

$$
\begin{aligned}
\chi_{v}=x^{(v)} \bar{v}+ & \sum_{\alpha \in \widehat{V}}\left(\sum_{j=1}^{\mu(\alpha)-1}\left(\sum_{C \in \mathscr{C}_{(\alpha)}^{v}} y_{j, f(C)}^{(\alpha)} \bar{C}^{j}\right)\right)+z^{(v)} C^{(V)} \\
+ & \sum_{\alpha \in \mathscr{A}_{\Gamma}^{V>1}}\left(\sum_{k=1}^{\operatorname{occ}(\alpha, V)-1}\left(\sum_{l=1}^{\mathrm{occ}(\alpha, V)}\left(\sum_{j=0}^{\mu(\alpha)-1} y_{j, f\left(C_{l}^{(\alpha)}\right), k}^{(\alpha)}\left(\overline{C_{l}^{(\alpha, v)}}\right)^{j} \overline{q_{l}^{(\alpha, v)}} \cdots \overline{q_{l+k-1}^{(\alpha, v)}}\right)\right)\right) \\
& +\sum_{\beta \in \mathscr{B}_{\Gamma}^{V}>1}\left(\sum_{k=1}^{\mathrm{occ}(\beta, V)-1}\left(\sum_{l=1}^{\mathrm{occ}(\beta, V)} y_{g\left(q_{l}^{(\beta, v)}\right), k}^{(\beta)} \overline{q_{l}^{(\beta, v)}} \cdots \overline{q_{l+k-1}^{(\beta, v)}}\right)\right)
\end{aligned}
$$

where $x^{(v)}, z^{(v)}, y_{j, f(C)}^{(\alpha)}, y_{j, f\left(C_{l}^{(\alpha)}\right), k}^{(\alpha)}, y_{g\left(q_{l}^{(\beta, v)}\right), k}^{(\beta)}$ are scalars from the field $K$. Let $f: \mathscr{C} \mathscr{C} \rightarrow \mathcal{Q}_{1}$ be the map that sends a special cycle to its first arrow, and $g: \neg \mathscr{C} \mathscr{C} \rightarrow \mathcal{Q}_{1}$ be the map that sends a non-special cycle to its first arrow. Furthermore, set

$$
\neg \mathscr{C} \mathscr{C}_{(\alpha)}=\bigcup_{V \in \mathscr{V}(\alpha)} \neg \mathscr{C}_{(\alpha)}^{v}
$$

for $\alpha \in \mathscr{D}_{\Gamma}$ and set

$$
\neg \mathscr{C} \mathscr{C}=\bigcup_{\alpha \in \mathscr{D}_{\Gamma}} \neg \mathscr{C} \mathscr{C}_{(\alpha)}
$$

The sets $\hat{V}, \mathscr{A}_{\Gamma}^{V>1}$ and $\mathscr{B}_{\Gamma}^{V>1}$ are defined in (2.5), (2.6) and (2.7) respectively. If $R_{1}=$ $\coprod_{a \in \mathcal{Q}_{1}} s(a) \Lambda t(a)$, let $d_{1}^{*}: R_{0} \rightarrow R_{1}$ be the $\Lambda$-bimodule morphism defined in Section 1.4. By 
expression (1.26) we know that the image of $\chi$ under $d_{1}^{*}$ can be computed as

$$
d_{1}^{*}(\chi)=\left(\chi_{s(a)} \bar{a}-\bar{a} \chi_{t(a)}\right)_{a \in \mathcal{Q}_{1}}
$$

Let $a \in \mathcal{Q}_{1}$ be an arrow of the quiver. By Proposition 2.2.1 there exists a unique $\alpha \in \Gamma_{0} \backslash \mathscr{T}_{\Gamma}$ associated to the arrow $a$ satisfying the properties of the proposition. For the vertex $\alpha$ we have the following possible cases.
(a) $\alpha \in \mathscr{A}_{\Gamma}$;
(b) $\alpha \in \mathscr{B}_{\Gamma}$;
(c) $\alpha \in \mathscr{C}_{\Gamma}$.

For the arrow $a \in \mathcal{Q}_{1}$ let $V, W$ be polygons of the configuration associated to the vertices $v, w$ of the quiver respectively, and suppose that $s(a)=v$ and $t(a)=w$. If $\alpha \in \mathscr{D}_{\Gamma}$ we can set $a=a_{i}^{(\alpha)}$, for some $1 \leq i \leq \operatorname{val}(\alpha)$, and the unique cycles $C, C^{\prime}$ of Proposition 2.2.1 are given respectively by

$$
\begin{aligned}
C_{s}^{(\alpha, v)} & =a_{i}^{(\alpha)} \cdots a_{i-1}^{(\alpha)}, \\
C_{r}^{(\alpha, w)} & =a_{i+1}^{(\alpha)} \cdots a_{i}^{(\alpha)},
\end{aligned}
$$

where $1 \leq s \leq \operatorname{occ}(\alpha, V)$ and $1 \leq r \leq \operatorname{occ}(\alpha, W)$. Using the graphical representation in Figure 2.5 we can represent the special $\alpha$-cycles $C_{s}^{(\alpha, v)}$ and $C_{r}^{(\alpha, w)}$ by

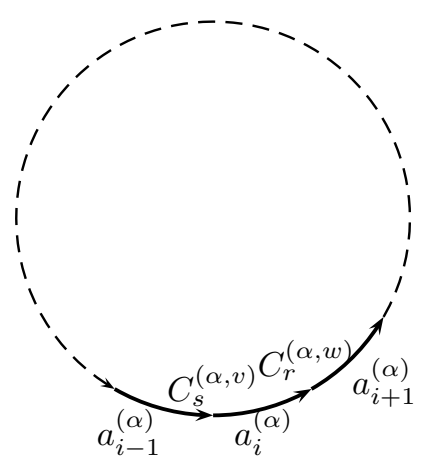

Figure 2.7

We start to examine the possible cases (a), (b) and (c) above.

- Case $\alpha \in \mathscr{A}_{\Gamma}$ : Using the generic expression in (2.29) for $\chi_{v}$ and $\chi_{w}$, and by Proposition 2.2.1 we have that the $a$-entry of (2.30) is given by

$$
\begin{aligned}
\left(x^{(v)}-x^{(w)}\right) \bar{a}+ & \sum_{j=1}^{\mu(\alpha)-1} y_{j, a_{i}^{(\alpha)}}^{(\alpha)}\left(\overline{C_{s}^{(\alpha, v)}}\right)^{j} \bar{a} \\
& +\sum_{l=1}^{\operatorname{occ}(\alpha, V)-1}\left(\sum_{j=0}^{\mu(\alpha)-1} y_{j, f\left(C_{s+l}^{(\alpha, v)}\right), \operatorname{occ}(\alpha, V)-l}^{(\alpha)}\left(\overline{C_{s+l}^{(\alpha, v)}}\right)^{j} q_{s+l}^{\overline{(\alpha, v)}} \cdots q_{s-1}^{(\alpha, v)} \bar{a}\right) \\
& -\left(\sum_{k=1}^{\operatorname{occ}(\alpha, W)-1}\left(\sum_{j=0}^{\mu(\alpha)-1} y_{j, a_{i+1}^{(\alpha)}, k}^{(\alpha)} \bar{a}\left(\overline{C_{r}^{(\alpha, w)}}\right)^{j} q_{r}^{\overline{(\alpha, w)}} \cdots q_{r+k-1}^{\overline{(\alpha, w)}}\right)+\sum_{j=1}^{\mu(\alpha)-1} y_{j, a_{i+1}^{(\alpha)}}^{(\alpha)} \bar{a}\left(\overline{C_{r}^{(\alpha, w)}}\right)^{j}\right)
\end{aligned}
$$


By Proposition 2.1.2(2) and Proposition 2.2.1(1a) we have

$$
\begin{aligned}
\left(C_{s+l}^{(\alpha, v)}\right)^{j} q_{s+l}^{(\alpha, v)} \cdots q_{s-1}^{(\alpha, v)} a & =q_{s+l}^{(\alpha, v)} \cdots q_{s-1}^{(\alpha, v)}\left(C_{s}^{(\alpha, v)}\right)^{j} a \\
a\left(C_{r}^{(\alpha, w)}\right)^{j} & =\left(C_{s}^{(\alpha, v)}\right)^{j} a
\end{aligned}
$$

for all $0 \leq j \leq \mu(\alpha)-1$, and all $1 \leq l \leq \operatorname{occ}(\alpha, V)-1$. So applying the relations in (2.31) and collecting similar terms we obtain

$$
\begin{aligned}
\left(x^{(v)}-x^{(w)}\right) \bar{a}+ & \sum_{l=1}^{\mathrm{occ}(\alpha, V)-1}\left(\sum_{j=0}^{\mu(\alpha)-1} y_{j, f\left(C_{s+l}^{(\alpha, v)}\right), \operatorname{occ}(\alpha, V)-l}^{(\alpha)} q_{s+l}^{\overline{(\alpha, v)}} \cdots q_{s-1}^{\overline{(\alpha, v)}}\left(\overline{C_{s}^{(\alpha, v)}}\right)^{j} \bar{a}\right) \\
& +\sum_{j=1}^{\mu(\alpha)-1}\left(y_{j, a_{i}^{(\alpha)}}^{(\alpha)}-y_{j, a_{i+1}^{(\alpha)}}^{(\alpha)}\right)\left(\overline{C_{s}^{(\alpha, v)}}\right)^{j} \bar{a}-\sum_{k=1}^{\operatorname{occ}(\alpha, W)-1}\left(\sum_{j=0}^{\mu(\alpha)-1} y_{j, a_{i+1}^{(\alpha)}, k}^{(\alpha)}\left(\overline{C_{s}^{(\alpha, v)}}\right)^{j} \bar{a} q_{r}^{\overline{(\alpha, w)}} \cdots q_{r+k-1}^{\overline{(\alpha, w)}}\right)
\end{aligned}
$$

Now, if $a=a_{i}^{(\alpha)}$ is a loop then we obtain that $q_{s}^{(\alpha, v)}=a, v=w, r=s+1$, and by Figure 2.7, $f\left(C_{s+1}^{(\alpha, v)}\right)=a_{i+1}^{(\alpha)}$. For the particular values $l=1$ and $k=\operatorname{occ}(\alpha, V)-1$ we can regroup terms in (2.32) which have same scalars. Then in (2.32) appears the expression

$$
\begin{aligned}
& \sum_{j=0}^{\mu(\alpha)-1} y_{j, a_{i+1}^{(\alpha)}, \mathrm{occ}(\alpha, V)-1}^{(\alpha)}\left(\left(\overline{C_{s+1}^{(\alpha, v)}}\right)^{j} q_{s+1}^{\overline{(\alpha, v)}} \cdots \overline{q_{s-1}^{(\alpha, v)}} \bar{a}-\left(\overline{C_{s}^{(\alpha, v)}}\right)^{j} \bar{a} q_{s+1}^{(\alpha, v)} \cdots q_{s-1}^{\overline{(\alpha, v)}}\right) \\
&=\sum_{j=0}^{\mu(\alpha)-2} y_{j, a_{i+1}^{(\alpha)}, \text { occ }(\alpha, V)-1}^{(\alpha)}\left(\left(\overline{C_{s+1}^{(\alpha, v)}}\right)^{j+1}-\left(\overline{C_{s}^{(\alpha, v)}}\right)^{j+1}\right)
\end{aligned}
$$

The right hand side of the equality in (2.33) holds because

- $\overline{C_{s+1}^{(\alpha, v)}}=q_{s+1}^{\overline{(\alpha, v)}} \cdots q_{s-1}^{\overline{(\alpha, v)}} \bar{a}$ and $\overline{C_{s}^{(\alpha, v)}}=\bar{a} q_{s+1}^{\overline{(\alpha, v)}} \cdots q_{s-1}^{\overline{(\alpha, v)}}$ by Proposition 2.1.2(1);

- $\left(\overline{C_{s+1}^{(\alpha, v)}}\right)^{\mu(\alpha)}=\left(\overline{C_{s}^{(\alpha, v)}}\right)^{\mu(\alpha)}$ by type one relations.

In conclusion, when $a=a_{i}^{(\alpha)}$ is a loop then (2.32) is equal to

$$
\begin{aligned}
& \sum_{j=1}^{\mu(\alpha)-1}\left(y_{j, a_{i}^{(\alpha)}}^{(\alpha)}-y_{j, a_{i+1}^{(\alpha)}}^{(\alpha)}\right)\left(\overline{C_{s}^{(\alpha, v)}}\right)^{j} \bar{a} \\
& +\sum_{\substack{1 \leq l \leq \text { occ }(\alpha, V)-1 \\
0 \leq j \leq \mu(\alpha)-1 \\
l \neq 1}} y_{j, f\left(C_{s+l}^{(\alpha)}\right), \operatorname{occ}(\alpha, V)-l} q_{s+l}^{\overline{(\alpha, v)}} \cdots \overline{q_{s-1}^{(\alpha, v)}}\left(\overline{C_{s}^{(\alpha, v)}}\right)^{j} \bar{a} \\
& -\sum_{\substack{1 \leq k \leq \text { occ }(\alpha, V)-1 \\
0 \leq j \leq \mu)-1 \\
k \neq 0,-1 \\
k \neq 0 \mathrm{occ}(\alpha, V)-1}} y_{j, a_{i+1}^{(\alpha)}, k}^{(\alpha)}\left(\overline{C_{s}^{(\alpha, v)}}\right)^{j} \bar{a} q_{s+1}^{\overline{(\alpha, v)}} \cdots \overline{q_{s+k}^{(\alpha, v)}} \\
& +\sum_{j=0}^{\mu(\alpha)-2} y_{j, a_{i+1}^{(\alpha)}, \operatorname{occ}(\alpha, V)-1}^{(\alpha)}\left(\left(\overline{C_{s+1}^{(\alpha, v)}}\right)^{j+1}-\left(\overline{C_{s}^{(\alpha, v)}}\right)^{j+1}\right)
\end{aligned}
$$

Note that expression obtained in (2.34) was to be expected. One of the missing scalars in (2.34) is exactly the same associated to the class of the element defined in (2.21) of Subsection 2.1.2, which is associated to the loop $q_{s}^{(\alpha, v)}=a_{i}^{(\alpha)}$ (the element associated to the scalar $\left.y_{\mu(\alpha)-1, a_{i+1}^{(\alpha)}, \operatorname{occ}(\alpha, V)-1}^{(\alpha)}\right)$. We showed in Proposition 2.1.7(2) that this type of elements are in the center of the algebra. 
- Case $\alpha \in \mathscr{B}_{\Gamma}$ : Using again the generic expression in (2.29) to compute $\chi_{v}$ and $\chi_{w}$, and Proposition 2.2.1, we have that the $a$-entry in this case is equal to

$$
\begin{aligned}
\left(x^{(v)}-x^{(w)}\right) \bar{a}+\sum_{l=1}^{\mathrm{occ}(\alpha, V)-1} y_{g\left(q_{s+l}^{(\alpha, v)}\right), \mathrm{occ}(\alpha, V)-l}^{(\alpha)} q_{s+l}^{\overline{(\alpha, v)}} \cdots q_{s-1}^{\overline{(\alpha, v)}} \bar{a} & \\
- & -\sum_{k=1}^{\mathrm{occ}(\alpha, W)-1} y_{a_{i+1}^{(\alpha)}, k}^{(\alpha)} \overline{\bar{a}} q_{r}^{\overline{(\alpha, w)}} \cdots \overline{q_{r+k-1}^{(\alpha, w)}}
\end{aligned}
$$

Also in this case, if $a=a_{i}^{(\alpha)}$ is a loop then $q_{s}^{(\alpha, v)}=a, v=w, r=s+1$ and $g\left(q_{s+1}^{(\alpha, v)}\right)=a_{i+1}^{(\alpha)}$. For the particular values $l=1$ and $k=\operatorname{occ}(\alpha, V)-1$ we have that in (2.35) appears the expression

$$
\begin{aligned}
& y_{a_{i+1}^{(\alpha), o c c}(\alpha, V)-1}^{(\alpha)} q_{s+1}^{\overline{(\alpha, v)}} \cdots q_{s-1}^{\overline{(\alpha, v)}} \bar{a}-y_{a_{i+1}^{(\alpha)}, \mathrm{occ}(\alpha, V)-1}^{(\alpha)} \bar{a} q_{s+1}^{\overline{(\alpha, v)}} \cdots q_{s-1}^{\overline{(\alpha, v)}} \\
& =y_{a_{i+1}^{(\alpha), o c c}(\alpha, V)-1}^{(\alpha)} q_{s+1}^{(\alpha, v)} \cdots q_{s}^{\overline{(\alpha, v)}}-y_{a_{i+1}^{(\alpha), o c c(\alpha, V)-1}}^{(\alpha)} q_{s}^{\overline{(\alpha, v)}} \cdots q_{s-1}^{\overline{(\alpha, v)}} \\
& =y_{a_{i+1}^{(\alpha)}, \operatorname{occ}(\alpha, V)-1}^{(\alpha)} \overline{C_{s+1}^{(\alpha, v)}}-y_{a_{i+1}^{(\alpha)}, \operatorname{occ}(\alpha, V)-1}^{(\alpha)} \overline{C_{s}^{(\alpha, v)}}=0 .
\end{aligned}
$$

This is due the fact that $\overline{C_{s+1}^{(\alpha, v)}}=\overline{C_{s}^{(\alpha, v)}}$ and Proposition 2.1.2(1). So, when $a=a_{i}^{(\alpha)}$ is a loop then (2.35) is equal to

$$
\sum_{l=2}^{\operatorname{occ}(\alpha, V)-1} y_{g\left(q_{s+l}^{(\alpha), ~}\right), \mathrm{occ}(\alpha, V)-l}^{(\alpha)} q_{s+l}^{\overline{(\alpha, v)}} \cdots q_{s}^{\overline{(\alpha, v)}} \quad-\quad \sum_{k=1}^{\operatorname{occ}(\alpha, V)-2} y_{a_{i+1}^{(\alpha)}, k}^{(\alpha)} q_{s}^{\overline{(\alpha, v)}} \cdots q_{s+k}^{\overline{(\alpha, v)}}
$$

Also in this case the expression obtained in (2.37) was to be expected. The missing scalar, $y_{a_{i+1}^{(\alpha)}(\alpha), o c c(\alpha, V)-1}^{(\alpha)}$, is associated to the class of the element defined in $(2.21)$, and this belongs to the center of the algebra.

- Case $\alpha \in \mathscr{C}_{\Gamma}$ : In this case the arrow $a$ is a loop, and by Proposition 2.1.7(1) the element $\bar{a}$ is in the center of the algebra. Then we have that the $a$-entry in (2.30) is equal to zero.

Observation 2.2.2. We give some remarks about the appearing terms in expressions (2.32), (2.34), (2.35) and (2.37). When the arrow $a$ is not a loop, the collection

$$
\begin{aligned}
\left\{\left(\overline{C_{s}^{(\alpha, v)}}\right)^{j} \bar{a}, q_{s+l}^{\overline{(\alpha, v)}} \cdots q_{s+\operatorname{occ}(\alpha, V)-1}^{\overline{(\alpha, v)}}\left(\overline{C_{s}^{(\alpha, v)}}\right)^{j} \bar{a},\left(\overline{C_{s}^{(\alpha, v)}}\right)^{j} \bar{a} q_{r}^{\overline{(\alpha, w)}} \cdots q_{r+k-1}^{\overline{(\alpha, w)}} \mid\right. \\
0 \leq j \leq \mu(\alpha)-1,1 \leq l \leq \operatorname{occ}(\alpha, V)-1,1 \leq k \leq \operatorname{occ}(\alpha, W)-1\}
\end{aligned}
$$

is a linearly independent subset of the $K$-space $\Lambda$. This is due the fact that the collection

$$
\left\{q_{s+l}^{\overline{(\alpha, v)}} \cdots q_{s-1}^{\overline{(\alpha, v)}}\left(\overline{C_{s}^{(\alpha, v)}}\right)^{j} \bar{a} \mid 0 \leq j \leq \mu(\alpha)-1,1 \leq l \leq \operatorname{occ}(\alpha, V)-1\right\}
$$

when it is not empty, is formed by paths strictly contained in $\left(C_{s}^{(\alpha, v)}\right)^{\mu(\alpha)} a$ which don't start at $a$ but they do finish at $a$; whereas the collection

$$
\left\{\left(\overline{C_{s}^{(\alpha, v)}}\right)^{j} \bar{a} q_{r}^{\overline{(\alpha, w)}} \cdots q_{r+k-1}^{\overline{(\alpha, w)}} \mid 0 \leq j \leq \mu(\alpha)-1,1 \leq k \leq \operatorname{occ}(\alpha, W)-1\right\}
$$

when it is not empty, is formed by prefixes of $\left(C_{s}^{(\alpha, v)}\right)^{\mu(\alpha)} a$ which start at a but they don't finish at $a$. Particularly, the intersection of (2.39) and (2.40) is empty. Elements of these sets 
correspond to the appearing terms in (2.32) and (2.35). When the arrow $a$ is a loop and $\alpha \in \mathscr{A}_{\Gamma}$, then the union of the sets

$$
\begin{gathered}
\left\{\left(\overline{C_{s}^{(\alpha, v)}}\right)^{j} \bar{a} \mid 1 \leq j \leq \mu(\alpha)-1\right\}, \\
\left\{q_{s+l}^{\overline{(\alpha, v)}} \cdots q_{s-1}^{\overline{(\alpha, v)}}\left(\overline{C^{(\alpha, v)}}\right)^{j} \bar{a} \mid 1<l \leq \operatorname{occ}(\alpha, V)-1,0 \leq j \leq \mu(\alpha)-1\right\}, \\
\left\{\left(\overline{C^{(\alpha, v)}} s\right)^{j} \bar{a} q_{s+1}^{(\alpha, v)} \cdots q_{s+k}^{(\alpha, v)} \mid 1 \leq k<\operatorname{occ}(\alpha, V)-1,0 \leq j \leq \mu(\alpha)-1\right\}, \\
\left\{\left(\overline{C_{s+1}^{(\alpha, v)}}\right)^{j+1}-\left(\overline{C_{s}^{(\alpha, v)}}\right)^{j+1} \mid 0 \leq j \leq \mu(\alpha)-2\right\},
\end{gathered}
$$

is a linearly independent subset of the $K$-space $\Lambda$. Elements of this set correspond to the appearing terms in (2.34). And when $\alpha \in \mathscr{B}_{\Gamma}$ also the set

$$
\left\{q_{s+l}^{\overline{(\alpha, v)}} \cdots q_{s}^{\overline{(\alpha, v)}}, q_{s}^{\overline{(\alpha, v)}} \cdots q_{s+k}^{\overline{(\alpha, v)}} \mid 1<l \leq \operatorname{occ}(\alpha, V)-1,1 \leq k<\operatorname{occ}(\alpha, V)-1\right\}
$$

is a linearly independent subset of the $K$-space $\Lambda$, and the elements in this set correspond to the appearing terms in (2.37).

Now we suppose that $\chi=\sum_{v \in \mathcal{Q}_{0}} \widetilde{\chi_{v}}$ is in $\operatorname{ker} d_{1}^{*}$. Then we have

$$
\chi_{s(a)} \bar{a}-\bar{a} \chi_{t(a)}=0 \text {, for all } a \in \mathcal{Q}_{1} .
$$

From expressions (2.32) and (2.35) and Observation 2.2.2 we see that we need that the scalar multiplying $\bar{a}$ is equal to

$$
x^{(s(a))}-x^{(t(a))}=0, \text { for all } a \in \mathcal{Q}_{1},
$$

for $\chi$ to be in $\operatorname{ker} d_{1}^{*}$. We are assuming that the configuration $\Gamma$ is connected then the induced quiver $\mathcal{Q}$ is connected, and since $\mathcal{Q}$ is formed by special cycles it follows that

$$
x^{(v)}=x^{(w)} \text {, for any } v, w \in \mathcal{Q}_{0} .
$$

But by (2.29) we see that this is equivalent to say that $\sum_{v \in \mathcal{Q}_{0}} \bar{v}=1_{\Lambda}$ is always in the center of $\Lambda$.

Now, let $\alpha \in \mathscr{A}_{\Gamma}$ and $\mathscr{A}_{(\alpha)}=\left\{a_{1}^{(\alpha)}, \ldots, a_{\mathrm{val}(\alpha)}^{(\alpha)}\right\}$ be the collection of arrows associated to $\alpha$, such that $t\left(a_{i}^{(\alpha)}\right)=s\left(a_{i+1}^{(\alpha)}\right)$ for each $1 \leq i \leq \operatorname{val}(\alpha)$, and where $a_{\mathrm{val}(\alpha)+1}^{(\alpha)}=a_{1}^{(\alpha)}$. For an arbitrary arrow $a_{i}^{(\alpha)}$ in $\mathscr{A}_{\mathscr{A}}(\alpha)$, by looking at (2.32) and (2.34) we see that if $a_{i}^{(\alpha)}$ is either a loop or not a loop we obtain the system of linear equations

$$
y_{j, a_{i}^{(\alpha)}}^{(\alpha)}-y_{j, a_{i+1}^{(\alpha)}}^{(\alpha)}=0, \text { for all } 1 \leq j \leq \mu(\alpha)-1
$$

That is, by considering each arrow in $\mathscr{A}_{(\alpha)}$ what we obtain is the following system of linear equations

$$
\begin{array}{cccccc}
y_{1, a_{1}^{(\alpha)}}^{(\alpha)}-y_{1, a_{2}^{(\alpha)}}^{(\alpha)} & = & 0, & \cdots, & y_{1, a_{\text {val }(\alpha)}^{(\alpha)}-y_{1, a_{1}^{(\alpha)}}^{(\alpha)}}^{(\alpha)} & = \\
y_{2, a_{1}^{(\alpha)}-y_{2, a_{2}^{(\alpha)}}^{(\alpha)}}^{(\alpha)} & = & 0, & \cdots, & y_{2, a_{\text {val }(\alpha)}^{(\alpha)}-y_{2, a_{1}^{(\alpha)}}^{(\alpha)}}^{(\alpha)} & = \\
\vdots & & & \vdots & \vdots \\
y_{\mu(\alpha)-1, a_{1}^{(\alpha)}}^{(\alpha)}-y_{\mu(\alpha)-1, a_{2}^{(\alpha)}}^{(\alpha)} & = & 0, & \cdots, & y_{\mu(\alpha)-1, a_{\text {val }(\alpha)}^{(\alpha)}-y_{\mu(\alpha)-1, a_{1}^{(\alpha)}}^{(\alpha)}}^{(\alpha)} & 0
\end{array}
$$


Then we have that this linear system gives us

$$
y_{j}^{(\alpha)}=y_{j, a_{i}^{(\alpha)}}^{(\alpha)}=y_{j, a_{i+1}^{(\alpha)}}^{(\alpha)}, \quad \forall 1 \leq i \leq \operatorname{val}(\alpha) .
$$

So, by returning to the expression (2.29) we see that

$$
\sum_{j=1}^{\mu(\alpha)-1}\left(\sum_{C \in \mathscr{C}_{\mathscr{C}}(\alpha)} y_{j}^{(\alpha)} \bar{C}^{j}\right)=\sum_{j=1}^{\mu(\alpha)-1} y_{j}^{(\alpha)}\left(\sum_{C \in \mathscr{C} \mathscr{C}_{(\alpha)}} \bar{C}^{j}\right)
$$

is an element of the center of the algebra, and by Observation 2.1.5 this element is equal to

$$
\sum_{j=1}^{\mu(\alpha)-1} y_{j}^{(\alpha)} C(\alpha)^{j}
$$

That is, for each $\alpha \in \mathscr{A}_{\Gamma}$ we have that the element in (2.52) is in the center of the algebra.

Observation 2.2.3. If $\alpha \in \mathscr{C}_{\Gamma}$ then there exists a unique polygon $V$ such that $\mathscr{V}_{(\alpha)}=\{V\}$ and $\mathscr{C} \mathscr{C}_{(\alpha)}=\left\{a^{(\alpha)}\right\}$. We showed in Section 2.1.2 that $\overline{a^{(\alpha)}} \in Z(\Lambda)$, then $\left(\overline{a^{(\alpha)}}\right)^{j} \in Z(\Lambda)$, for every $1 \leq j \leq \mu(\alpha)-1$. So, by defining $C(\alpha)=\overline{a^{(\alpha)}}$, for each $\alpha \in \mathscr{C}_{\Gamma}$, we have that

$$
\bigcup_{\alpha \in \mathscr{A}_{\Gamma} \cup \mathscr{C}_{\Gamma}}\left\{C(\alpha)^{j} \mid 1 \leq j \leq \mu(\alpha)-1\right\}
$$

is contained in $Z(\Lambda)$.

Recall that we are assuming that $\chi$ is in $\operatorname{ker} d_{1}^{*}$. Consider the case $\alpha \in \mathscr{A}_{\Gamma}$ with $\mathscr{A}_{(\alpha)}=$ $\left\{a_{1}^{(\alpha)}, \ldots, a_{\mathrm{val}(\alpha)}^{(\alpha)}\right\}$ the collection of arrows associated to $\alpha$. By Observation 2.2.2 and expressions (2.32) and (2.34) we have that for each $1 \leq i \leq \operatorname{val}(\alpha)$

- if $a_{i}^{(\alpha)}$ is not a loop then

$$
y_{j, a_{i+1}^{(\alpha)}, k}^{(\alpha)}=0, \quad \begin{aligned}
& \forall 0 \leq j \leq \mu(\alpha)-1 \\
& \forall 1 \leq k \leq \operatorname{occ}(\alpha, W)-1
\end{aligned}
$$

- if $a_{i}^{(\alpha)}$ is a loop then

$$
y_{j, a_{i+1}^{(\alpha)}, k}^{(\alpha)}=0, \quad \begin{aligned}
& \forall 0 \leq j \leq \mu(\alpha)-1 \\
& \forall 1 \leq k<\operatorname{occ}(\alpha, W)-1
\end{aligned}
$$

and

$$
y_{j, a_{i+1}^{(\alpha)}, \operatorname{occ}(\alpha, V)-1}^{(\alpha)}=0, \forall 0 \leq j \leq \mu(\alpha)-2 .
$$

From this we see that the only scalars that are not zero are those of the form

$$
y_{\mu(\alpha)-1, a_{i+1}^{(\alpha)}, \mathrm{occ}(\alpha, V)-1}^{(\alpha)}
$$

when $a_{i}^{(\alpha)}$ is a loop, which are associated to the cycles defined in (2.21). Now, if $\alpha \in \mathscr{B}_{\Gamma}$ then by Observation 2.2.2 and expressions (2.35) and (2.37) we have that for each $1 \leq i \leq \operatorname{val}(\alpha)$ 
- if $a_{i}^{(\alpha)}$ is not a loop

$$
y_{a_{i+1}^{(\alpha)}, k}^{(\alpha)}=0, \forall 1 \leq k \leq \operatorname{occ}(\alpha, W)-1
$$

- if $a_{i}^{(\alpha)}$ is a loop then

$$
y_{a_{i+1}^{(\alpha)}, k}^{(\alpha)}=0, \forall 1 \leq k<\operatorname{occ}(\alpha, V)-1
$$

Also at this case we see that the only scalars that are not zero are those of the form

$$
y_{a_{i+1}^{(\alpha)}(\alpha)}^{(\alpha)}(\alpha, V)-1
$$

when $a_{i}^{(\alpha)}$ is a loop, which also are associate to the cycles in (2.21).

Finally getting back to expression in (2.29) and applying each of the previous computations, if $\chi=\sum_{v \in \mathcal{Q}_{0}}$ is in $\operatorname{ker}_{1}^{*}$ then $\chi$ must be a linear combination of the elements of the following sets

- $\left\{1_{\Lambda}\right\}$

- $\bigcup_{\alpha \in \mathscr{A}_{\Gamma} \cup \mathscr{C}_{\Gamma}}\left\{C(\alpha)^{j} \mid 1 \leq j \leq \mu(\alpha)-1\right\}$

- $\left\{C^{(V)} \mid V \in \Gamma_{1}\right\}$

- $\bigcup_{\alpha \in h^{-1}(1)}\left\{\overline{D_{V, s}^{(\alpha)}} \mid(V, s) \in \mathscr{M}^{(\alpha)}\right\}$

where $h$ is the map defined in (2.18) and $\mathscr{M}^{(\alpha)}$ the set defined in (2.20). The union of all sets above is disjoint and it is a subset of the $K$-basis of $R_{0}=\coprod_{v \in \mathcal{Q}_{0}} v \Lambda v$ generating $\operatorname{ker} d_{1}^{*}$. Hence is a $K$-basis of $Z(\Lambda)$.

Recall that we are assuming that the Brauer configuration $\Gamma$ is reduced and connected and $\operatorname{rad}^{2}(\Lambda) \neq 0$. For the induced quiver $\mathcal{Q}$ of the Brauer configuration $\Gamma$, let $\# \operatorname{Loops}(\mathcal{Q})$ denote the number of loops in the quiver $\mathcal{Q}$, then it is not difficult to prove that

$$
\# \operatorname{Loops}(\mathcal{Q})=\sum_{\alpha \in h^{-1}(1)}\left(\sum_{V \in \mathscr{V}_{(\alpha)}}\left|\Psi_{(\alpha)}^{v}\right|\right)+\left|\mathscr{C}_{\Gamma}\right|
$$

See $(2.20),(2.21)$ and $(2.22)$ to check. We can say that the dimension of $Z(\Lambda)$ is equal to

$$
1+\sum_{\alpha \in \mathscr{A}_{\Gamma} \cup \mathscr{C}_{\Gamma}}(\mu(\alpha)-1)+\left|\Gamma_{1}\right|+\sum_{\alpha \in h^{-1}(1)}\left(\sum_{V \in \mathscr{V}_{(\alpha)}}\left|\Psi_{(\alpha)}^{v}\right|\right) .
$$

Using the fact that $\mu(\alpha)-1=0$ for every $\alpha \in \mathscr{B}_{\Gamma} \cup \mathscr{T}_{\Gamma}$ and the expression in (2.54), the dimension can be expressed as

$$
\begin{aligned}
& 1+\sum_{\alpha \in \mathscr{A}_{\Gamma} \cup \mathscr{B}_{\Gamma} \cup \mathscr{C}_{\Gamma}}(\mu(\alpha)-1)+\left|\Gamma_{1}\right|+\# \operatorname{Loops}(\mathcal{Q})-\left|\mathscr{C}_{\Gamma}\right| \\
= & 1+\sum_{\alpha \in \Gamma_{0}}(\mu(\alpha)-1)+\left|\Gamma_{1}\right|+\# \operatorname{Loops}(\mathcal{Q})-\left|\mathscr{C}_{\Gamma}\right| \\
= & 1+\sum_{\alpha \in \Gamma_{0}} \mu(\alpha)+\left|\Gamma_{1}\right|-\left|\Gamma_{0}\right|+\# \operatorname{Loops}(\mathcal{Q})-\left|\mathscr{C}_{\Gamma}\right|
\end{aligned}
$$


Finally we obtain the desired result.

Theorem 2.2.4. Let $\Gamma$ be a reduced and connected Brauer configuration, let $\mathcal{Q}$ be its induced quiver and let $\Lambda$ be the induced Brauer configuration algebra such that $\operatorname{rad}^{2}(\Lambda) \neq 0$. Then

$$
\operatorname{dim}_{K} Z(\Lambda)=1+\sum_{\alpha \in \Gamma_{0}} \mu(\alpha)+\left|\Gamma_{1}\right|-\left|\Gamma_{0}\right|+\# \operatorname{Loops}(\mathcal{Q})-\left|\mathscr{C}_{\Gamma}\right|,
$$

where $\mathscr{C}_{\Gamma}=\left\{\gamma \in \Gamma_{0} \mid \operatorname{val}(\gamma)=1\right.$ and $\left.\mu(\gamma)>1\right\}$.

Let's see some examples.

Example 2.2.5. Consider the Brauer configuration algebra $\Lambda$ induced by the Brauer configuration $\Gamma$ of the Example 1.3.10. Because the combinatorial data of the configuration $\Gamma$ we have that $\mathscr{A}_{\Gamma}=\Gamma_{0}$. Then by watching the quiver in (1.7) the elements of the $K$-basis of $Z(\Lambda)$ are given by

$$
\left\{\overline{1}_{\Lambda}\right\} \cup\left\{C^{\left(V_{1}\right)}, C^{\left(V_{2}\right)}, C^{\left(V_{3}\right)}, C^{\left(V_{4}\right)}\right\} \cup\left\{C(i)^{l} \mid 1 \leq i \leq 3,1 \leq l \leq N-1\right\},
$$

where

$$
C(i)=\overline{a_{1}^{(i)} a_{2}^{(i)} a_{3}^{(i)}}+\overline{a_{2}^{(i)} a_{3}^{(i)} a_{1}^{(i)}}+\overline{a_{3}^{(i)} a_{1}^{(i)} a_{2}^{(i)}},
$$

for each $1 \leq i \leq 3$, and

$$
\begin{aligned}
& \left.C^{\left(V_{1}\right)}=\overline{\left(a_{1}^{(1)} a_{2}^{(1)} a_{3}^{(1)}\right.}\right)^{N}, \\
& \left.C^{\left(V_{2}\right)}=\overline{\left(a_{2}^{(2)} a_{3}^{(2)} a_{1}^{(2)}\right.}\right)^{N}, \\
& \left.C^{\left(V_{3}\right)}=\overline{\left(\bar{a}_{2}^{(3)} a_{3}^{(3)} a_{1}^{(3)}\right.}\right)^{N}, \\
& \left.C^{\left(V_{4}\right)}=\overline{\left(a_{2}^{(1)} a_{3}^{(1)} a_{1}^{(1)}\right.}\right)^{N} .
\end{aligned}
$$

The dimension of the center is equal to

$$
\begin{aligned}
\operatorname{dim}_{K} Z(\Lambda) & =1+3 N+4-3+0-0 \\
& =3 N+2
\end{aligned}
$$

Example 2.2.6. Consider now the Brauer configuration algebra $\Lambda^{\prime}$ induced by the Brauer configuration $\Gamma^{\prime}$ of the Example 1.3.10. In this Brauer configuration we have that $\mathscr{B}_{\Gamma}=\Gamma_{0}^{\prime}$, and by watching the quiver in (1.8) we see that there are loops. Let's consider the cyclic drawing of the quiver to represent the special and the non special cycles. So, for the vertex $\alpha$ we have

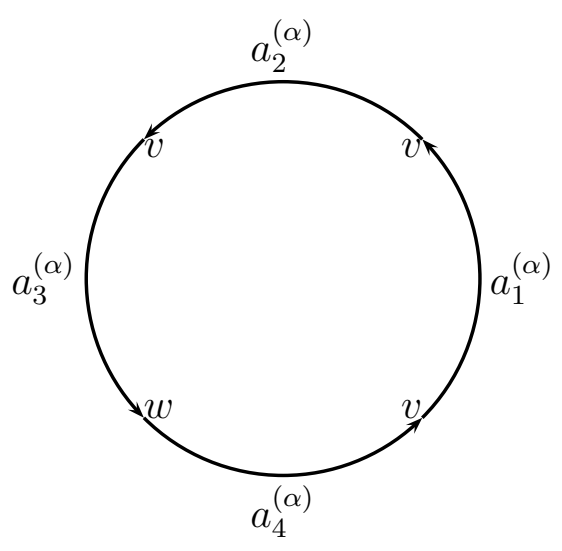

Figure 2.8 
Setting

$$
q_{1}^{(\alpha, v)}=a_{1}^{(\alpha)}, q_{2}^{(\alpha, v)}=a_{2}^{(\alpha)}, q_{3}^{(\alpha, v)}=a_{3}^{(\alpha)} a_{4}^{(\alpha)}
$$

we see that the colection $\mathscr{M}^{(\alpha)}$ defined in (2.20) is equal to

$$
\mathscr{M}^{(\alpha)}=\{(V, 1),(V, 2)\},
$$

then by $(2.21)$

$$
\begin{aligned}
& D_{V, 1}^{(\alpha)}=q_{2}^{(\alpha, v)} q_{3}^{(\alpha, v)}=a_{2}^{(\alpha)} a_{3}^{(\alpha)} a_{4}^{(\alpha)}, \\
& D_{V, 2}^{(\alpha)}=q_{3}^{(\alpha, v)} q_{1}^{(\alpha, v)}=a_{3}^{(\alpha)} a_{4}^{(\alpha)} a_{1}^{(\alpha)} .
\end{aligned}
$$

Now, for the vertex $\beta$ we have the cyclic drawing

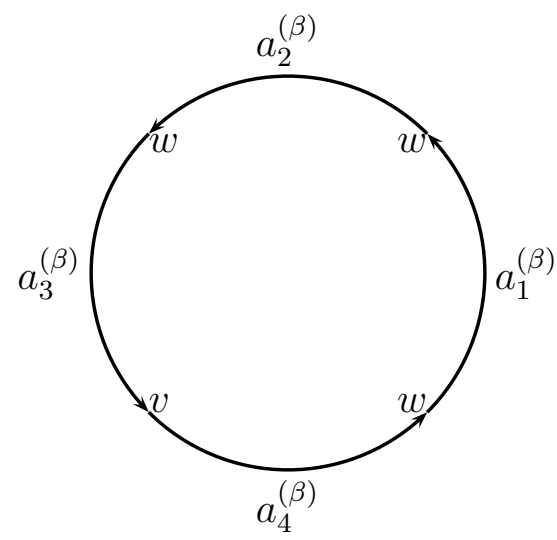

Figure 2.9

If we set

$$
q_{1}^{(\beta, v)}=a_{1}^{(\beta)}, q_{2}^{(\beta, v)}=a_{2}^{(\beta)}, q_{3}^{(\beta, v)}=a_{3}^{(\beta)} a_{4}^{(\beta)}
$$

we obtain that

$$
\mathscr{M}^{(\beta)}=\{(W, 1),(W, 2)\}
$$

and

$$
\begin{aligned}
& D_{W, 1}^{(\beta)}=q_{2}^{(\beta, w)} q_{3}^{(\beta, w)}=a_{2}^{(\beta)} a_{3}^{(\beta)} a_{4}^{(\beta)}, \\
& D_{W, 2}^{(\beta)}=q_{3}^{(\beta, w)} q_{1}^{(\beta, w)}=a_{3}^{(\beta)} a_{4}^{(\beta)} a_{1}^{(\beta)} .
\end{aligned}
$$

Then the $K$-basis of the center of $\Lambda^{\prime}$ is given by

$$
\left\{\overline{1}_{\Lambda^{\prime}}\right\} \cup\left\{C^{(V)}, C^{(W)}\right\} \cup\left\{\overline{D_{V, 1}^{(\alpha)}}, \overline{D_{V, 2}^{(\alpha)}}, \overline{D_{W, 1}^{(\beta)}}, \overline{D_{W, 2}^{(\beta)}}\right\},
$$

with

$$
\begin{aligned}
C^{(V)} & =\overline{a_{1}^{(\alpha)} a_{2}^{(\alpha)} a_{3}^{(\alpha)} a_{4}^{(\alpha)}}, \\
C^{(W)} & =\overline{a_{1}^{(\beta)} a_{2}^{(\beta)} a_{3}^{(\beta)} a_{4}^{(\beta)}},
\end{aligned}
$$

and the dimension is equal to

$$
\begin{aligned}
\operatorname{dim}_{K} Z\left(\Lambda^{\prime}\right) & =1+2+2-2+4-0 \\
& =7
\end{aligned}
$$

Example 2.2.7. Now consider the Brauer configuration algebra $\Lambda^{(4)}$ induced by the Brauer 
configuration $\Gamma^{(4)}$ of the Example 2.1.9. By the quiver in (2.27) we have that $H=\{\alpha, \beta, \gamma\}=$ $h^{-1}(1)=\mathscr{B}_{\Gamma^{(4)}}$, then by setting

$$
\begin{aligned}
& q_{1}^{\left(\alpha, w_{1}\right)}=a_{1}^{(\alpha)}, \quad q_{2}^{\left(\alpha, w_{1}\right)}=a_{2}^{(\alpha)}, \\
& q_{1}^{\left(\beta, w_{2}\right)}=a_{1}^{(\beta)}, \quad q_{2}^{\left(\beta, w_{2}\right)}=a_{2}^{(\beta)}, \\
& q_{1}^{\left(\gamma, w_{3}\right)}=a_{1}^{(\gamma)}, \quad q_{2}^{\left(\gamma, w_{3}\right)}=a_{2}^{(\gamma)},
\end{aligned}
$$

we obtain that

$$
\begin{aligned}
& D_{W_{1}, 1}^{(\alpha)}=a_{2}^{(\alpha)}, \quad D_{W_{1}, 2}^{(\alpha)}=a_{1}^{(\alpha)}, \\
& D_{W_{2}, 1}^{(\beta)} a_{2}^{(\beta)}, \quad D_{W_{2}, 2}^{(\beta)}=a_{1}^{(\beta)}, \\
& D_{W_{3}, 1}^{(\gamma)}=a_{2}^{(\gamma)}, \quad D_{W_{3}, 2}^{(\gamma)}=a_{1}^{(\gamma)} .
\end{aligned}
$$

The $K$-basis of $Z\left(\Lambda^{(4)}\right)$ is then given by the collection

$$
\left\{\overline{1}_{\Lambda^{(4)}}\right\} \cup\left\{C^{\left(W_{1}\right)}, C^{\left(W_{2}\right)}, C^{\left(W_{3}\right)}\right\} \cup\left\{\overline{D_{W_{1}, 1}^{(\alpha)}}, \overline{D_{W_{1}, 2}^{(\alpha)}}, \overline{D_{W_{2}, 1}^{(\beta)}}, \overline{D_{W_{2}, 2}^{(\beta)}}, \overline{D_{W_{3}, 1}^{(\gamma)}}, \overline{D_{W_{3}, 2}^{(\gamma)}}\right\},
$$

where

$$
\begin{aligned}
& C^{\left(W_{1}\right)}=\overline{a_{1}^{(1)} a_{2}^{(1)} a_{3}^{(1)}}=\overline{a_{1}^{(\alpha)} a_{2}^{(\alpha)}}, \\
& C^{\left(W_{2}\right)}=\overline{a_{2}^{(1)} a_{3}^{(1)} a_{1}^{(1)}}=\overline{a_{1}^{(\beta)} a_{2}^{(\beta)}}, \\
& C^{\left(W_{3}\right)}=\overline{a_{3}^{(1)} a_{1}^{(1)} a_{2}^{(1)}}=\overline{a_{1}^{(\gamma)} a_{2}^{(\gamma)}} .
\end{aligned}
$$

As we see the quiver in (2.27) we have that the number of loops is equal to 6 , then using the dimension formula of the Theorem 2.2.4 it follows that

$$
\begin{aligned}
\operatorname{dim}_{K} Z\left(\Lambda^{(4)}\right) & =1+4+3-4+6-0 \\
& =10
\end{aligned}
$$

which coincides with the number of elements in the $K$-basis above.

Recall the definitions of the map $h$ in (2.19) and the set $H$ in (2.25). The following proposition gives us multiplicative relations between the elements of the basis of the center of a Brauer configuration algebra.

Proposition 2.2.8. Let $\Gamma$ be a reduced and connected Brauer configuration and let $\Lambda$ be the induced Brauer configuration algebra such that $\operatorname{rad}^{2}(\Lambda) \neq 0$. The followings relations are verified in $\Lambda$ :

- $C(\alpha) C(\beta)=0, \forall \alpha, \beta \in \mathscr{A}_{\Gamma}, \alpha \neq \beta$;

- $C(\gamma) C(\theta)=0, \forall \gamma, \theta \in \mathscr{C}_{\Gamma}, \gamma \neq \theta$

- $C(\alpha) C(\gamma)=0, \forall \alpha \in \mathscr{A}_{\Gamma}, \forall \gamma \in \mathscr{C}_{\Gamma}$

- $C^{(V)} C^{(W)}=0, \forall V, W \in \Gamma_{1}$;

- $C^{(V)} C(\alpha)=C^{(V)} C(\gamma)=0, \forall V \in \Gamma_{1}, \forall \alpha \in \mathscr{A}_{\Gamma}, \forall \gamma \in \mathscr{C}_{\Gamma}$.

(2) If $\alpha \in h^{-1}(1)$ and $(V, s) \in \mathscr{M}^{(\alpha)}$ then

$$
\overline{D_{V, s}^{(\alpha)}} C\left(\alpha^{\prime}\right)=\overline{D_{V, s}^{(\alpha)}} C^{(V)}=0, \forall \alpha \in \mathscr{A}_{\Gamma} \cup \mathscr{C}_{\Gamma}, \forall V \in \Gamma_{1} .
$$

(3) Let $\alpha, \beta \in h^{-1}(1)$ and let $(V, s) \in \mathscr{M}^{(\alpha)},(W, t) \in \mathscr{M}^{(\beta)}$. 
- if $\alpha, \beta \notin H$ then

$$
\overline{D_{V, s}^{(\alpha)}} \overline{D_{W, t}^{(\beta)}}=0
$$

- if $\alpha \notin H$ and $\beta \in H$ then

$$
\overline{D_{V, s}^{(\alpha)}} \overline{D_{W, t}^{(\beta)}}=0
$$

- if $\alpha, \beta \in H$ and $\alpha \neq \beta$ then

$$
\overline{D_{V, s}^{(\alpha)}} \overline{D_{W, t}^{(\beta)}}=0
$$

- if $\alpha, \beta \in H$ and $\alpha=\beta$ then

$$
\overline{D_{V, s}^{(\alpha)}} \overline{D_{V, t}^{(\alpha)}}=C^{(V)},
$$

when $s=1$ and $t=2$ or $s=2$ and $t=1$, and

$$
{\overline{D_{V, s}^{(\alpha)}}}^{2}=0,
$$

for $s=1,2$.

Proof. Relations in (1) and (2) are easily checked. Also the first three items in (3) are easily checked, and the last item is proved in Proposition 2.1.8.

For the next example we are going to use some rudiments and tools from the commutative theory of Gröbner bases. We refer the reader to [AL94, Chapter 1] to consult definitions and results.

Example 2.2.9. Let $\Gamma^{\prime \prime \prime}$ be the Brauer configuration of Example 1.3.14. The induced quiver of this Brauer configuration is given by

$$
a_{2}^{(1)} C_{1} a_{1}^{(1)}
$$

We see that in this configuration $\mathscr{B}_{\Gamma^{\prime \prime \prime}}=\Gamma_{0}^{\prime \prime \prime}=\{1\}=H$. By setting $q_{1}^{(1, v)}=a_{1}^{(1)}$ and $q_{2}^{(1, v)}=a_{2}^{(1)}$ we have then that

$$
\begin{aligned}
& D_{V, 1}^{(1)}=q_{2}^{(1, v)}=a_{2}^{(1)} \\
& D_{V, 2}^{(1)}=q_{1}^{(1, v)}=a_{1}^{(1)} .
\end{aligned}
$$

If $\Lambda^{\prime \prime \prime}$ is the Brauer configuration algebra induced by $\Gamma^{\prime \prime \prime}$, then a $K$-basis of its center is given

$$
\left\{\overline{1}_{\Lambda^{\prime \prime \prime}}\right\} \cup\left\{C^{(V)}\right\} \cup\left\{\overline{D_{V, 1}^{(1)}}, \overline{D_{V, 2}^{(1)}}\right\}
$$

and the number of elements in this set obviously coincides with the dimension formula of Theorem 2.2.4, i.e, $\operatorname{dim}_{K} Z\left(\Lambda^{\prime \prime \prime}\right)=1+1+1-1+2-0=4$. Now, consider the commutative polynomial ring $K\left[x_{1}, x_{2}, x_{3}\right]$ with the term order given by the lexicographical order (see [AL94, Definition 1.4.2]) and such that $x_{1}>x_{2}>x_{3}$.

Let $\varphi: K\left[x_{1}, x_{2}, x_{3}\right] \rightarrow Z\left(\Lambda^{\prime \prime \prime}\right)$ be the unique ring homomorphism given by the $K$-linear map

$$
\begin{aligned}
& x_{1} \longmapsto \frac{C^{(V)}}{D_{V, 1}^{(1)}} \\
& x_{2} \longmapsto \frac{D_{V, 2}^{(1)}}{x_{3}}
\end{aligned}
$$


By relations in Proposition 2.2.8 we easily see that

$$
\left\{x_{1}^{2}, x_{2}^{2}, x_{3}^{2}, x_{1} x_{2}, x_{1} x_{3}, x_{1}-x_{2} x_{3}\right\} \subset \operatorname{ker} \varphi .
$$

If $I$ is the ideal of $K\left[x_{1}, x_{2}, x_{3}\right]$ generated by $\left\{x_{1}^{2}, x_{2}^{2}, x_{3}^{2}, x_{1} x_{2}, x_{1} x_{3}, x_{1}-x_{2} x_{3}\right\}$, then by [AL94, Theorem 1.7.4] it follows that $\rho$ is a Gröbner basis for the ideal $I$. If we set $\rho=$ $\left\{x_{2}^{2}, x_{3}^{2}, x_{1}-x_{2} x_{3}\right\}$ then it is easy to prove that $\rho$ is a reduced Gröbner basis for $I$. Assume that $I \varsubsetneqq \operatorname{ker} \varphi$, then there exists a polynomial $f \in \operatorname{ker} \varphi$ such that $f \notin I$. By [AL94, Theorem 1.6.7] there exist polynomials $g_{1}, g_{2}, g_{3}$ and a unique polynomial $r \neq 0$ such that

$$
f=x_{2}^{2} g_{1}+x_{3}^{2} g_{2}+\left(x_{1}-x_{2} x_{3}\right) g_{3}+r,
$$

and $r$ is reduced with respect to $\rho$ (see [AL94, Definition 1.5.5]). So, by the lexicographical order on the polynomial ring it follows that the polynomial $r$ must be of the form

$$
r=a_{1} x_{2}+a_{2} x_{3}
$$

for scalar $a_{1}, a_{2}$ of the field $K$. Applying the ring homomorphism $\varphi$ to the expression in (??) we obtain

$$
a_{1} \overline{D_{V, 1}^{(1)}}+a_{2} \overline{D_{V, 2}^{(1)}}=0,
$$

but the equality above implies that $a_{1}=a_{2}=0$, which in turn implies that $f \in I$, a contradiction. So, we have that

$$
\begin{aligned}
K\left[x_{1}, x_{2}, x_{3}\right] /\left\langle x_{2}^{2}, x_{3}^{2}, x_{1}-x_{2} x_{3}\right\rangle & \cong K\left[x_{2}, x_{3}\right] /\left\langle x_{2}^{2}, x_{3}^{2}\right\rangle \\
& \cong Z\left(\Lambda^{\prime \prime \prime}\right)
\end{aligned}
$$

as rings, which is what we expect, because as we mentioned in Example 1.3.14 the algebra $\Lambda^{\prime \prime \prime}$ is commutative and isomorphic to $K\left[x_{1}, x_{2}\right] /\left\langle x_{1}^{2}, x_{2}^{2}\right\rangle$. 
CENTER AND DIMENSION 


\section{Bibliography}

[AL94] William W. Adams e Philippe. Loustaunau. An introduction to gröbner bases, volume 3. Graduate Studies in Math, AMS, 1994. 54, 55

[ARS97] Maurice Auslander, Idum Reiten e Sverre Smalø. Representation theory of artin algebras, volume 35. Cambridge Studies in Advanced Mathematics, 1997. 5

[ASS06] Ibrahim Assem, Daniel Simson e Andrzej Skowronski. Elements of the representation theory of associative algebras: 1, volume 65. London Math. Society ST, 2006. 5,7

[Bar01] Michael J. Bardzell. Noncommutative groebner bases and hochschild cohomology. Contemp. Math.,, 286:227-240, 2001. 30

[BM98] Michael J. Bardzell e Eduardo N. Marcos. Induced boundary maps for the cohomology of monomial and auslander algebras. Em CMS Conf. Proc., volume 24, páginas 47-54, 1998. 2, 30

[DF78] P. W. Donovan e M.-R. Freislich. The indecomposable modular representation of certain groups with dihedral sylow subgroup. Math. Ann., 238:207-216, 1978. 1

[Dro] Ju Drozd. Tame and wild matrix problems. Em Representation Theory, II (Proc. Second Internat. Conf., Carleton Univ., Ottawa, Ont., 1979). Lecture Notes in Math. Springer, Berlin-New York. 1

[GSa] Edward L. Green e Sibylle Schroll. Brauer configuration algebras: A generalization of brauer graph algebras. arXiv: 1508.03617. 1, 2, 12, 16, 20, 21, 27

[GSb] Edward L. Green e Sibylle Schroll. Special multiserial algebras, brauer configuration algebras and more: a survey. arXiv: 1703.01408. 2

[GS04] Edward L. Green e Nicole Snashall. Projective bimodule resolutions of an algebra and vanishing of the second cohomology group. Forum Math., 16:17-36, 2004. 30

[GS16] Edward L. Green e Sibylle Schroll. Multiserial and special multiserial algebras and their representations. Advances in Mathematics, 302:1111-1136, 2016. 2

[GS17] Edward L. Green e Sibylle Schroll. Special multiserial algebras are quotients of symmetric special multiserial algebras. J. of Algebra, 473:397-405, 2017. 2

[GSST] Edward L. Green, Sibylle Schroll, Nicole Snashall e Rachel Taillefer. The Ext algebra of a brauer graph algebra. to appear Journal of Non-commutative Geometry, arXiv: 1302.6413. 1, 24 
[HHJW84] Von Höhne, Hans-Joachim e Josef Waschbüsch. Die struktur $n$-reihiger algebren. Comm. Algebra, 12:1187-1206, 1984. 2

[Jan69] G. J. Janusz. Indecomposable modules for finite groups. Ann. of Math., (2) 89:209-241, 1969. 1

[Rog] K. W. Roggenkamp. Biserial algebras and graphs (English summary). Em Algebras and Modules, II (Geiranger, 1996). Amer. Math. Soc. 2

[Sch] Sibylle Schroll. Brauer graph algebras, a survey. arXiv: 1612.00061. 1, 2

[Sch14] Ralf Schiffler. Quiver Representations. CMS Books in Mathematics, SpringerVerlag, 2014. 5 